\title{
Linear pre-metric electrodynamics and deduction of the light cone
}

\author{
Inaugural-Dissertation \\ zur \\ Erlangung des Doktorgrades \\ der Mathematisch-Naturwissenschaftlichen Fakultät \\ der Universität zu Köln
}

vorgelegt von

Guillermo F. Rubilar A.

aus Concepción, Chile

Köln 2002 
Berichterstatter:

Tag der mündlicher Prüfung:
Prof. Dr. Friedrich W. Hehl

Prof. Dr. Claus Kiefer

10.06.2002 
to Alejandra 



\section{Zusammenfassung}

Diese Arbeit beschäftigt sich mit den Grundlagen der klassischen Elektrodynamik, mit ihrer grundlegenden mathematischen Struktur, mit den elektromagnetischen Eigenschaften der Raumzeit und, insbesondere, mit den notwendigen Bedingungen, die von dem elektromagnetischen Medium erfüllt werden müssen, damit eine Lichtkegelstruktur folgt.

In den Kapiteln 2 und 3 stellen wir die allgemeingültige Struktur der Elektrodynamik in einem beliebigen Medium dar. Ein wichtiges Ergebnis dieser Untersuchung ist die allgemeine Ableitung der Fresnel'schen Gleichung, die die lokalen Eigenschaften der Lichtfortpflanzung beschreibt. Es ist uns gelungen, eine allgemein-kovariante Ableitung der Fresnel'schen Gleichung für ein beliebiges Medium mit linearer Konstitutivrelation zu geben. Diese Entwicklungen sind nützlich im Zusammenhang mit den folgenden drei Aspekten: 1) Sie stellen die grundlegende Struktur der klassischen Elektrodynamik transparent dar; 2) Sie können als Grundlage für allgemeinere Modelle der Raumzeit und deren elektromagnetischen Eigenschaften betrachtet werden. Mögliche Anwendungen des Formalismus auf Test-Theorien sind Beispiele dafür; 3) Der in den Kapiteln 2 und 3 entwickelte Formalismus kann auch im Bereich der klassischen Optik als eine allgemein kovariante Theorie der Elektrodynamik in inhomogenen, anisotropen, und dissipativen materiellen Medien interpretiert und benutzt werden.

Im Kapitel 4 untersuchen wir den speziellen Fall, in dem ein Lichtkegel vorhanden ist, und die Bedingungen dafür, dass eine solche Struktur möglich ist. Insbesondere werden wir den Zusammenhang zwischen den unter bestimmten Bedingungen vom Konstitutivtensor definierten dualen Operatoren und der Existenz einer konformen Metrik untersuchen. Es stellt sich heraus, dass die sogenannte Abgeschlossenheitsrelation und die Symmetrie des Konstitutivtensors hinreichende Bedingungen für die Existenz einer konformen Metrik sind. Wir werden auch untersuchen, wie die Metrik-Komponenten von 
den Komponenten des Konstitutivtensors abgeleitet werden können, wenn die zwei oben genannten Bedingungen erfüllt sind. Wir werden auch eine alternative und einfachere Ableitung der Metrik-Komponenten geben, die auf der direkten Anwendung der allgemeinen Ergebnisse über die Fresnel'sche Gleichung basiert. Am Ende dieser Arbeit werden wir dieselbe Methode anwenden, um die Auswirkungen der Lockerung der Symmetrie-Bedingung, und wie sie die Existenz des Lichtkegels beeinflusst, zu untersuchen. 


\section{Abstract}

This thesis deals with the foundations of classical electrodynamics, its fundamental mathematical structure, the electromagnetic properties of spacetime and, in particular, the conditions necessarily to be satisfied by a electromagnetic medium such that it induces a lightcone structure.

In chapters 2 and 3 , we formulate a general framework for electrodynamics in an arbitrary linear medium. An important result of this study is the derivation of the Fresnel equation describing the local properties of the propagation of electromagnetic waves. We were able to give a generally covariant derivation of the Fresnel equation for an arbitrary medium with linear constitutive law. These developments are useful at least in three different aspects. First, they make the fundamental structures of classical electrodynamics more transparent. Second, they may provide a basis for a deeper understanding and for generalized models of spacetime and its electromagnetic properties. This includes for instance the application of the formalism to study test theories. Third, the formalism developed in chapters 2 and 3 can be also interpreted and used in optics as a general covariant theory of electrodynamics in inhomogeneous, anisotropic, and in general dissipative, material media.

Finally, in chapter 4 we study the particular case in which a light cone structure is induced, and the circumstances under which such a structure emerges. In particular, we will study the relationship between dual operators defined by the constitutive tensor under certain conditions and the existence of a conformal metric. Closure and symmetry of the constitutive tensor will be found to be conditions which ensure the existence of a conformal metric. We will also see how the metric components can be explicitly derived from the constitutive tensor if these two conditions are satisfied. We will also give an alternative, simpler, and more physical derivation of the metric, based on direct use of our general results about the Fresnel equation describing the 
local properties of light propagation. Finally, we will apply the same method to explore the consequences of relaxing the condition of symmetry and how this affects the emergence of the light cone. 


\section{Contents}

Zusammenfassung i

$\begin{array}{ll}\text { Abstract } & \text { iii }\end{array}$

1 Introduction $\mathbf{1}$

1.1 Motivation ..................... 1

1.2 Maxwell-Lorentz equations in vector notation ...... 5

1.3 Electrodynamics in a material medium . . . . . . . . 6

1.4 Maxwell-Lorentz equations in 3+1 exterior form notation . . . 8

1.5 Poincaré covariant Maxwell equations . . . . . . . . . . . 9

1.6 Poincaré group invariance and 'natural' invariance . . . . . . 10

1.7 Maxwell equations in curved spacetime . . . . . . . . 11

2 Electrodynamics on an arbitrary 4D-manifold 13

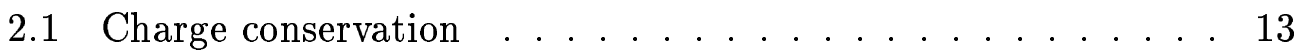

2.2 Lorentz force . . . . . . . . . . . . . . 16

2.3 Magnetic flux conservation . . . . . . . . . . . . 17

2.4 'Space'-'time' Decomposition . . . . . . . . . . . . . 18

2.5 Measuring $H \ldots \ldots \ldots . \ldots . \ldots . \ldots 22$

2.6 Constitutive relations . . . . . . . . . . . . 24

2.6.1 Linear constitutive relations . . . . . . . . . 27

2.6.2 Nonlinear constitutive relations ......... . 34

2.6.3 Effective constitutive tensor . . . . . . . . . 35

2.7 Symmetries and Energy-momentum . . . . . . . . . . . . 37

2.7.1 Symmetry of a linear medium . . . . . . . . . . 37

2.7.2 Conservation of energy-momentum . . . . . . . . 39

2.7.3 Axiom 4: Energy-momentum tensor . . . . . . . . 44 
3 Wave propagation $\quad 47$

3.1 Propagation of singularities . . . . . . . . . . . . . 47

3.2 Fresnel equation . . . . . . . . . . . . . 50 50

3.2.1 Coordinate $3+1$ decomposition of the Fresnel equation 53

3.2.2 Properties of the Fresnel tensor density . . . . . . . 54

3.3 Light rays . . . . . . . . . . . . . . 5 56

3.3.1 Fresnel equation for $V \ldots \ldots \ldots 58$

3.3.2 Particular cases . . . . . . . . . . . 59

3.4 Application to propagation of perturbations in media with nonlinear constitutive relations .......... 60

4 Light cone structure $\quad \mathbf{6 3}$

4.1 Looking for metric-independent conditions . . . . . . . . 64

4.2 Reciprocity and closure . . . . . . . . . . . . 6 65

4.3 Dual operators and metrics . . . . . . . . . 67

4.3.1 Peres's early work . . . . . . . . . . . 69

4.3.2 Schönberg-Urbantke formula . . . . . . . . 75

4.3.3 Necessary and sufficient conditions for the constitutive tensor ................ 87

4.4 General solution of the Closure Relation . . . . . . . . 88

4.4.1 Explicit derivation of the metric components from the constitutive tensor . . . . . . . . . 9 90

4.4.2 Properties of the metric . . . . . . . . . . 92

4.4.3 Alternative derivation ............ 92

4.5 Relaxing the symmetry condition . . . . . . . . . . 94

5 Conclusions and Prospects $\quad 99$

5.1 A possible dynamical theory for the constitutive tensor ? . . . 101

$\begin{array}{ll}\text { A The situation so far } & 105\end{array}$

$\begin{array}{ll}\text { B Electrodynamics in a material medium } & 107\end{array}$

$\begin{array}{lr}C \text { Some exterior and tensor calculus } & 109\end{array}$

C.1 Tensor and tensor densities . . . . . . . . . . . 109

C.2 Lie derivative . . . . . . . . . . . . . 110

C.3 Differential forms ................... 111

C.4 Exterior derivative . . . . . . . . . . . . 112 
C.5 Interior product of a vector and a $p$-form . . . . . . . 112

C.6 Lie derivative of a $p$-form . . . . . . . . . . . . 113

C.7 Twisted and untwisted forms . . . . . . . . . 113

C.8 Hodge dual operator . . . . . . . . . . . . . . 114

$\begin{array}{ll}\text { D Local Lorentz invariance } & \mathbf{1 1 5}\end{array}$

$\begin{array}{ll}\text { E Computer Algebra } & \mathbf{1 1 7}\end{array}$

E.1 Defining the components of the constitutive tensor . . . . . 117

E.2 Computing the Fresnel tensor . . . . . . . . . . . 118

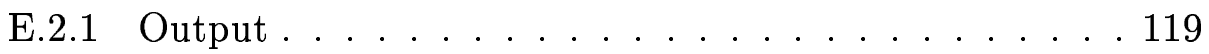

E.3 Checking equations (3.41) . . . . . . . . . 120

E.4 Defining the 3-dimensional constitutive matrices . . . . . . 121

E.5 Fresnel equation in terms of 3-dimensional constitutive matrices 123

E.6 Checking equations $(3.30)-(3.37) \ldots . . . . . . . .124$

$\begin{array}{ll}\text { Acknowledgements } & 135\end{array}$ 


\section{Chapter 1}

\section{Introduction}

\subsection{Motivation}

In Einstein's theory of gravity, General Relativity (GR), the fundamental variable describing the gravitational field is the spacetime metric. Einstein's equations are partial differential equations which, together with suitable boundary conditions, determine the metric field from the energy distribution of matter. The metric tensor defines, on the one side, the causal structure of spacetime: it allows to distinguish between timelike, spacelike, and null vectors. On the other hand, a metric tensor also determines the length of any worldline. The length of a timelike worldline is interpreted as proper time, i.e. the time measured by standard clocks when transported along that timelike curve. This was called by Synge the 'chronometric hypothesis' [87]. In Einstein's theory the metric determines additionally the affine properties of spacetime. The causal properties are, however, also of an electromagnetic nature. Light rays are integral lines of null vectors fields, null hypersurfaces are the regions through which electromagnetic waves can propagate. From an operational point of view it is clear that most of our knowledge of spacetime is extracted from the properties of electromagnetic fields and how they propagate. Today, we send electromagnetic waves towards (reflecting) objects to measure distances. We measure velocities by means of Doppler-shifted electromagnetic waves, etc. The properties of spacetime itself are then defined as those independent of the specific configuration of the fields and interpreted as describing the underlying 'substrate' on which we make measurements.

From the ten independent components of a metric tensor (in a given coor- 
dinate system), nine define the causal (or conformal) structure. This means, nine out of then components of the metric are related to electromagnetic properties of spacetime.

In the last decades the idea that GR cannot be the fundamental theory of gravity and spacetime has gained much support. It is known that quantizing GR leads to a quantum theory which is not renormalizable. This means that the theory is inconsistent (at least perturbatively) at high energies. Therefore, it is important to seek for different approaches to understand every aspect of GR and to explore possibilities of how to generalize the concepts on which it is build on. Some promising alternative approaches have been developed, which lead to generalized theories or to alternative formulations or interpretations of GR. As an example, gauge theories of gravity have been proposed, which seem to be in a better position to be unified with the other three fundamental interactions, and found under suitable conditions to have GR as limit. Most alternative theories include additional degrees of freedom, apart from those corresponding to the spacetime metric.

My goal here is not to propose a concrete alternative spacetime theory, but the more modest one of studying a more general framework describing classical electrodynamics in which a metric tensor is not assumed as a basic fundamental field from the very beginning. This will raise the question of which fundamental structures of classical electrodynamics can be formulated without a metric and for which developments it is indispensable. The framework will be called pre-metric (or sometimes metric-free) electrodynamics, and it shares many features and analogies with the theory of electrodynamics in a material medium. By studying such a general framework, the conformal properties of spacetime, i.e., the conformal spacetime metric with its nine independent components, can eventually emerge as an special case under certain particular circumstances. The study of the conditions under which a conformal metric structure is induced is also one of the subjects of this work. This framework could then be useful to develop a generalized theory of spacetime in which the properties of vacuum are treated in analogy to a material medium and in which GR which its causal structure could be recovered in suitable limiting cases.

The idea of considering the spacetime metric as a secondary, derived field, is not new. Already in 1921 Eddington studied a 'purely affine gravity' model, in which the metric is defined in terms of a symmetric affine connection, see [79]. Thus, in Eddingston's theory, the causal properties of spacetime are derived from its affine properties. Other models have proposed to replace 
the metric by a trio of self-dual 2 -forms $[8,9]$. In [10] the metric is obtained from a solution of a theory formulated only in terms of a $S L(2, C)$ connection, a tetrad, and a scalar density. More recently, it has been shown by Barceló et al. [2, 3] that an effective metric can be derived (defined) for almost any lagrangian theory of scalar fields, provided one considers perturbations of the fields around some background configuration. If the theory depends of a single scalar field, then a metric can be uniquely defined (so that the equation for the field perturbation can be written as a Klein-Gordon equation with respect to that metric) [2]. If more scalar fields are involved, multiple metrics can be introduced in general, which can be described as refringence (birefringence, trirefringence, ...) in the sense that different fields of the theory would 'see' different effective metric structures [3]. In these models, the causal properties of space are again a manifestation of the dynamics of some more fundamental fields.

In a related context, there has been renewly interest in recent years for so-called 'analogue models of gravity', see [99]. These models are based on the results that some condensed matter systems, as for instance acoustic perturbations in a moving fluid, light in a moving dielectric, or quasiparticles in a moving superfluid, can be described in term of some 'effective metric'. The metric is also here a derived, secondary object which turns out to be useful for a geometrical description (à la GR) of the system properties and depends on the more fundamental degrees of freedom of the system. The interest in these analogue models lies in the possibility to construct systems in which kinematical properties of physics in curved space could be simulated and tested in the laboratory. In [80], for instance, the possibility constructing 'dielectric black hole analogs' is discussed, i.e. dielectric materials in which the effective metric describes the analog of an event horizon, see also [47, 98] Dynamics, on the other hand is not likely to be simulated, since in general the dynamics of the effective metric can be completely different to that imposed by Einstein's equations in GR.

The axiomatic approach to classical electrodynamics presented here has been developed from the original ideas of Kottler [42] and van Dantzig [97]. They seem to be the firsts who recognized that the fundamental structure of Maxwell's equations is independent of the metric and affine structure of spacetime. In what concerns the derivation of the spacetime metric from linear electrodynamics, in which we are interested here, Peres [68], already in 1962, wrote: 'It is therefore suggested to consider the electromagnetic field as fundamental, and the metric field only as a subsidiary quantity'. This same 
idea was also developed by Toupin [92] and by Schönberg [77] who showed that a conformal metric structure is induced assuming that the 'constitutive tensor' defining the 'spacetime relation' between electromagnetic field excitations and strengths (see section 2.6) satisfies the conditions of symmetry and closure (section 4). Jadczyk [36] also showed that a spacetime metric can be introduced under the above mentioned conditions. However, only very recently and explicit derivation of the induced conformal metric has been given by Obukhov and Hehl [63].

In chapters 2 and 3, we formulate a general framework for electrodynamics in an arbitrary linear medium ${ }^{1}$. These developments are useful at least in three different aspects. First, they make the fundamental structures of classical electrodynamics more transparent. Second, they may provide a basis for a deeper understanding and for generalized models of spacetime and its electromagnetic properties. This includes for instance the application of the formalism to study test theories, see for instance [25] and references therein. Third, the formalism developed in chapters 2 and 3 can be also interpreted and used in optics as a general covariant theory of electrodynamics in inhomogeneous, anisotropic, and in general dissipative, material media.

Finally, in chapter 4 we study the particular case in which a light cone structure is induced, and the circunstances under which such structure emerges. In particular, we will study the relationship between dual operators defined by the constitutive tensor under certain conditions and the existence of a conformal metric. Closure and symmetry of the constitutive tensor will be found to be conditions which ensure the existence of a conformal metric. We will also see how the metric components can be explicitly derived from the constitutive tensor if these two conditions are satisfied. We will also give an alternative, simpler, and more physical derivation of the metric, based on direct use of our general results about the Fresnel equation describing the local properties of light propagation. Finally, we will apply the same method to explore the consequences of relaxing the condition of symmetry and how this affects the emergence of the light cone.

\footnotetext{
${ }^{1}$ We use the word 'medium' in a general sense, to refer to any 'arena' on which electromagnetic phenomena could take place. We, in particular, include the vacuum as a particular 'medium'. On the other hand, 'material' or 'material medium' will be used to refer to media with a known (atomic) substructure, as for instance, crystal, liquids, etc.
} 


\subsection{Maxwell-Lorentz equations in vector no- tation}

We start with electrodynamics within the framework of Special Relativity (SR).

In the usual 3-dimensional vector notation, $\mathbf{V}:=\left(\mathbf{V}^{\mathbf{x}}, \mathbf{V}^{\mathbf{y}}, \mathbf{V}^{\mathbf{z}}\right)$, the Maxwell-Lorentz equations in integral form read:

$$
\begin{aligned}
& \int_{\partial V} \mathbf{D} \cdot d \mathbf{S}=\int_{V} \tilde{\rho} d V, \quad \oint_{\partial S} \mathbf{H} \cdot d \mathbf{r}=\int_{S} \mathbf{J} \cdot d \mathbf{S}+\frac{d}{d t}\left(\int_{S} \mathbf{D} \cdot d \mathbf{S}\right) \\
& \int_{\partial V} \mathbf{B} \cdot d \mathbf{S}=0, \quad \oint_{\partial S} \mathbf{E} \cdot d \mathbf{r}=-\frac{d}{d t}\left(\int_{S} \mathbf{B} \cdot d \mathbf{S}\right) .
\end{aligned}
$$

Here $\mathbf{D}$ is the electric excitation (historically called 'electric displacement'), $\widetilde{\rho}$ the electric charge density, $\mathbf{H}$ the magnetic excitation (historically called 'magnetic field'), $\mathbf{J}$ the electric current density, $\mathbf{B}$ the magnetic field strength and $\mathbf{E}$ the electric field strength. The integrals above are defined over arbitrary volumes $V$ with boundary $\partial V$ and over arbitrary surfaces $S$ with boundary $\partial S$, respectively. The corresponding volume elements of the three, two-, and one-dimensional regions are denoted by $d V, d \mathbf{S}$, and $d \mathbf{r}$. Finally, the dot - denotes the 3 -dimensional scalar product of vectors, which is a metric-dependent object.

Equation (1.1a) summarizes the Gauss law. It implies that the field lines defined by $\mathbf{D}$ can be open, the ends of which are located at points where the charge is located. Equation (1.2a) is usually interpreted as expressing the absence of magnetic monopoles in nature ${ }^{2}$. It implies that the magnetic lines defined by B must be closed. Equation (1.2b) summarizes Faraday's induction law ('a time-variation of an magnetic field induces an electric field').

The field strengths $\mathbf{E}$ and $\mathbf{B}$ are operationally defined by means of the Lorentz force law. On test charges, the force density is given by

$$
\mathbf{F}=\tilde{\rho} \mathbf{E}+\mathbf{J} \times \mathbf{B} .
$$

Here $\times$ is the 3 -dimensional vector product, also a metric-dependent object.

The Maxwell equations are completed by the relations

$$
\mathbf{D}=\varepsilon_{0} \mathbf{E}, \quad \mathbf{H}=\frac{1}{\mu_{0}} \mathbf{B} .
$$

\footnotetext{
${ }^{2}$ For recent (unsuccessful) searches for magnetic monopoles, see $[26,1,37]$ and references therein.
} 
The constants $\varepsilon_{0}$ and $\mu_{0}$ are called permittivity and permeability of vacuum, respectively. The speed of light, i.e., the speed with which electromagnetic perturbations propagate, is given by $c=\frac{1}{\sqrt{\epsilon_{0} \mu_{0}}}$.

As it is well known, the Maxwell equations (1.1) and (1.2) can be written in differential form as

$$
\begin{array}{ll}
\nabla \cdot \mathbf{D}=\tilde{\rho}, & \nabla \times \mathbf{H}=\mathbf{J}+\frac{\partial \mathbf{D}}{\partial t} \\
\nabla \cdot \mathbf{B}=0, & \nabla \times \mathbf{E}=-\frac{\partial \mathbf{B}}{\partial t} .
\end{array}
$$

The inhomogeneous Maxwell equations (1.5) are such that the conservation of electric charge is automatically satisfied, i.e.

$$
\frac{\partial \widetilde{\rho}}{\partial t}+\nabla \cdot \mathbf{J}=0
$$

Actually, Maxwell completed the electromagnetic equations known at his time by adding the 'electric displacement' term $\frac{\partial \mathbf{D}}{\partial t}$ such that the resulting equations were consistent with charge conservation.

\subsection{Electrodynamics in a material medium}

It is well known that a macroscopic description of electromagnetic phenomena inside a material medium (treated as a continuum) can be achieved using macroscopic Maxwell equations which are of the same form as (1.5) and (1.6), but were now

- $\mathbf{D}$ and $\mathbf{H}$ denote macroscopic field strengths,

- the sources $\rho$ and $\mathbf{J}$ are now external charge and current densities, respectively, and

- the relation between $(\mathbf{D}, \mathbf{H})$ and $(\mathbf{E}, \mathbf{B})$ is now not given by (1.4) but by a constitutive relations

$$
\mathbf{D}=\mathbf{D}[\mathbf{E}, \mathbf{B}], \quad \mathbf{H}=\mathbf{H}[\mathbf{E}, \mathbf{B}]
$$

which contain the information of the particular electromagnetic properties of the medium under consideration. 
Among the many possible particular cases (nonlocal constitutive laws, ...) we recall here the case of a linear anisotropic medium for which the constitutive relations are usually written, in components (see for instance [45], page 313), as

$$
D_{a}=\varepsilon_{a b} E_{b}+\alpha_{a b} B_{b}, \quad H_{a}=\mu_{a b}^{-1} B_{b}+\beta_{a b} E_{b} .
$$

Here $a, b, \ldots=1,2,3, \varepsilon_{a b}$ is the permittivity tensor, and $\mu_{a b}$ the permeability tensor of the medium. The tensors $\alpha$ and $\beta$ describe magneto-electrical properties. For examples of and further discussions on magneto-electric media, see $[65]$.

Consider the case in which $\alpha_{a b}$ and $\beta_{a b}$ vanish. A non-magnetic medium corresponds to the subcase in which $\mu_{a b}=\mu_{0} \delta_{a b}$ so that the vacuum relation $(1.4 \mathrm{~b})$ holds. Interesting properties of non-magnetic anisotropic media regarding propagation of plane electromagnetic waves include [45]:

- In general, for a triaxial crystal (i.e. when the three eigenvalues of $\epsilon_{a b}$ are different), a fourth order Fresnel equation determines the dispersion relation of plane waves.

- The wave vector $\mathbf{k}$ and the ray vector $\mathbf{s}$ are in general not parallel: $\mathbf{k}$ is the vector normal to the wave front, $\mathbf{s}$ is the direction of energy propagation.

- Birefringence in uniaxial crystals (two eigenvalues of $\epsilon_{a b}$ are equal): the Fresnel equation factorizes into two quadratic factors, one isotropic factor corresponding to 'ordinary' waves and an anisotropic one corresponding to 'extraordinary' waves.

Effects analogous to the above mentioned will be discussed in chapter 3 . Other examples of local constitutive laws include:

- Double refraction induced by an electric field. This so-called Kerr effect can be induced if an isotropic material is placed in a constant electric field. The electric field breaks the isotropy of the medium producing a change in the dielectric constant, leading to effects similar to those observed in uniaxial crystals. This effect can be described by the nonlinear constitutive law corresponding to the following dielectric tensor:

$$
\varepsilon_{a b}=\varepsilon_{0} \delta_{a b}+\alpha E_{a} E_{b},
$$

with some constant $\alpha$. 


\begin{tabular}{|c||c|c|c|c|c|c|}
\hline vector/scalar & $\widetilde{\rho}$ & $\mathbf{J}$ & $\mathbf{H}$ & $\mathbf{D}$ & $\mathbf{E}$ & $\mathbf{B}$ \\
\hline$p$-form & $\rho$ & $j$ & $\mathcal{H}$ & $\mathcal{D}$ & $E$ & $B$ \\
\hline$p$ & 3 & 2 & 1 & 2 & 1 & 2 \\
\hline
\end{tabular}

Table 1.1: Correspondence between vectors and exterior forms.

- Magneto-optical effects: The dielectric constant depends on the magnetic field strength $\mathbf{H}$.

\subsection{Maxwell-Lorentz equations in $3+1$ exte- rior form notation}

From the Maxwell-Lorentz equations in their integral form, see (1.1) and (1.2), one can see that the different fields appear associated to integrals over regions of different dimensionality. For the sources, we see that $\tilde{\rho}$ is integrated over 3-dimensional regions (volumes) while $\mathbf{J}$ is integrated over surfaces, i.e. 2-dimensional regions. The field excitations $\mathbf{H}$ and $\mathbf{D}$ are integrated over 1- and 2-dimensional domains, respectively. Finally, the field strengths $\mathbf{E}$ and $\mathbf{B}$ appears in the Maxwell-Lorentz equations under 1- and 2-dimensional integrals, respectively. From the theory of exterior forms, see for instance [18] and appendix C.4, we know that a $p$-form is the natural object to be integrated over a $p$-dimensional domain. This means that the Maxwell-Lorentz equations can be reformulated in terms of exterior forms according to the identifications of table 1.1. In terms of exterior forms, the Maxwell-Lorentz equations (1.1) and (1.2) are naturally expressed as

$$
\begin{array}{ll}
\underline{d} \mathcal{D}=\rho, & \underline{d} \mathcal{H}=\frac{\partial}{\partial t} \mathcal{D}+j, \\
\underline{d} B=0, & \underline{d} E+\frac{\partial}{\partial t} B=0 .
\end{array}
$$

Here $\underline{d}=d x^{a} \wedge \partial_{a}$ denotes the 3-dimensional exterior derivative (we will use $d$ for the 4-dimensional exterior derivative). Note the internal consistency of these equations, since $\underline{d}$ increases by one the rank of the exterior form on which it is applied. Conversely, taking the equations (1.11) and (1.12) as 
starting point, one can derive the corresponding field equations in terms of field components. We decompose each form as follows:

$$
\begin{aligned}
& \rho=\frac{1}{3 !} \rho_{a b c} d x^{a} \wedge d x^{b} \wedge d x^{c}=\tilde{\rho} d x \wedge d y \wedge d z, \\
& j=\frac{1}{2} j_{a b} d x^{a} \wedge d x^{b}=\frac{1}{2} \hat{\epsilon}_{a b c} j^{c} d x^{a} \wedge d x^{b}, \\
& \mathcal{H}=\mathcal{H}_{a} d x^{a}, \quad \mathcal{D}=\frac{1}{2} \mathcal{D}_{a b} d x^{a} \wedge d x^{b}=\frac{1}{2} \hat{\epsilon}_{a b c} \mathcal{D}^{c} d x^{a} \wedge d x^{b}, \\
& E=E_{a} d x^{a}, \quad B=\frac{1}{2} B_{a b} d x^{a} \wedge d x^{b}=\frac{1}{2} \hat{\epsilon}_{a b c} B^{c} d x^{a} \wedge d x^{b},
\end{aligned}
$$

Here $\hat{\epsilon}_{a b c}=\hat{\epsilon}_{[a b c]}$, with $\hat{\epsilon}_{123}=1$, is the 3 -dimensional Levi-Civita symbol. Then, using (1.13)-(1.16) into the equations (1.11) and (1.12) one directly finds, see for instance [78],

$$
\begin{array}{rlrl}
\partial_{a} \mathcal{D}^{a} & =\rho, & \epsilon^{a b c} \partial_{b} \mathcal{H}_{c} & =\frac{\partial}{\partial t} \mathcal{D}^{a}+j^{a}, \\
\partial_{a} B^{a} & =0, & \epsilon^{a b c} \partial_{b} E_{c}+\frac{\partial}{\partial t} B^{a}=0
\end{array}
$$

which generalize (1.5) and (1.6).

However, the formulation in terms of exterior forms has the advantage that the corresponding $p$-forms are independent of the 3-dimensional coordinate system used. In other words (1.11) and (1.12), and therefore also (1.17) and (1.18), are valid not only in cartesian coordinates but in any 3-dimensional coordinate system. Actually, if one considers the exterior forms in (1.13)-(1.16) as basic field variables, and we do here, then equations (1.11) and (1.12) are independent of any metric or affine structure of the 3-dimensional space.

\subsection{Poincaré covariant Maxwell equations}

As usually shown in textbooks on SR, the Maxwell equations (1.5) and (1.6) can be written covariantly under Poincaré transformations by defining the field strength $F_{i j}=-F_{j i}(i, j, \ldots=t, x, y, z)$ by

$$
F_{t x}:=-E^{x}, \quad F_{t y}:=-E^{y}, \quad F_{t z}:=-E^{z},
$$




$$
\begin{array}{llrl}
F_{x y}:=B^{z}, & F_{y z}:=B^{x}, & F_{z x}:=B^{y}, \\
H_{t x}:=H^{x}, & H_{t y}:=H^{y}, & H_{t z}:=H^{z}, \\
H_{x y}:=D^{z}, & H_{y z}:=D^{x}, & H_{z x}:=D^{y} .
\end{array}
$$

Additionally, the electric current 4 -vector density $\mathcal{J}^{i}$ is defined as

$$
\mathcal{J}^{t}:=\rho, \quad \mathcal{J}^{x}:=J^{x}, \quad \mathcal{J}^{y}:=J^{y}, \quad \mathcal{J}^{z}:=J^{z} .
$$

Then, we can write (1.5) and (1.6) as

$$
\begin{aligned}
& \frac{1}{2} \epsilon^{i j k l} \partial_{j} H_{k l}=\mathcal{J}^{i}, \\
& \epsilon^{i j k l} \partial_{j} F_{k l}=0,
\end{aligned}
$$

where $\epsilon^{i j k l}$ is the Levi-Civita symbol. See appendix C.1. The relation between $F_{i j}$ and $H_{i j}$, namely equation (1.4), is translated into

$$
H_{i j}=\frac{1}{2} \sqrt{\frac{\varepsilon_{0}}{\mu_{0}}} \hat{\epsilon}_{i j k l} \eta^{k m} \eta^{l n} F_{m n},
$$

where $\eta^{i j}$ are the components of the Minkowski metric in cartesian coordinates: $\eta_{i j}=\operatorname{diag}\left(c^{2},-1,-1,-1\right), \eta^{i j}=\operatorname{diag}\left(c^{-2},-1,-1,-1\right)$. Inserting (1.26) into (1.24) we obtain (always in cartesian coordinates) the inhomogeneous Maxwell equations in a form which is often used in SR, namely

$$
\sqrt{\frac{\varepsilon_{0}}{\mu_{0}}} \partial_{j} F^{i j}=\mathcal{J}^{i}
$$

\subsection{Poincaré group invariance and 'natural' invariance}

It is well known that the Maxwell equations (1.24), (1.25) and (1.26) are form invariant under Lorentz transformations of the form

$$
x^{i} \rightarrow x^{i^{\prime}}:=\Lambda^{i^{\prime}}{ }^{i},
$$

with $\Lambda^{i^{\prime}}{ }_{i} \in S O(1,3)$, i.e. satisfying

$$
\Lambda_{i}^{i^{\prime}} \Lambda_{j}^{j^{\prime}} \eta^{i j}=\eta^{i^{\prime} j^{\prime}}
$$


This form invariance of the Maxwell equations means that the physics of the electromagnetic fields remains the same on rotated frames and in frames moving with constant velocity with respect to each other (i.e. boosted frames).

However, a more careful analysis shows that the field equations (1.24) and (1.25) are actually form invariant under any coordinate transformation $x^{i} \rightarrow$ $x^{i^{\prime}}=x^{i^{\prime}}\left(x^{i}\right)$, provided $F_{i j}$, and $H_{i j}$ are considered as components of a second order (antisymmetric) tensor field, and $\mathcal{J}^{i}$ as the components of a vector density field of weight +1 . This feature of (1.24) and (1.25) is sometimes called 'natural invariance' of the Maxwell equations, see [72], chapter 3, for an extended discussion. The natural invariance shows, on the other hand, that the physical information about the equivalence of frames under Lorentz transformations is contained exclusively in the spacetime relation between field excitation and field strength (1.26). This is a property of the vacuum. Furthermore, we note that the Minkowski metric appears only in (1.26). Looking at (1.26) it is then clear that this invariance of the vacuum is a direct consequence of the form invariance of the Minkowski metric with respect to Lorentz transformations, see (1.29).

\subsection{Maxwell equations in curved spacetime}

The traditional recipe to construct a theory including the interaction with the gravitational field is to 'replace partial derivatives by covariant derivatives', also called 'minimal coupling' to the gravitational field. This procedure ensures that the resulting equations are covariant under an arbitrary change of coordinates, see, for instance, [54] for more details and examples. The procedure is not free of ambiguities when one considers the electromagnetic potential as fundamental variable, since then the Maxwell equations are of second order and then a 'normal ordering' problem appears when applying the recipe above. This is a consequence of the fact that covariant derivatives do not commute in a curved spacetime.

A not widely recognized fact is however, that in the case of electrodynamics, the mentioned recipe is completely unnecessary since the Maxwell equations, when properly formulated, are 'naturally covariant', as we have seen in section 1.6. For the (rather trivial) transition from a Minkowski space to a Riemannian space with metric $g$ one just needs to replace the spacetime 
relation by

$$
H_{i j}=\sqrt{\frac{\varepsilon_{0}}{\mu_{0}}} \hat{\epsilon}_{i j k l} \sqrt{|g|} g^{k m} g^{l n} F_{m n}
$$

with $g:=\operatorname{det}\left(g_{i j}\right)$, so that $F$ and $H$ are tensors, as required by the natural invariance of (1.24) and (1.25). At every event one can find riemannian coordinates which will reduce the metric to its minkowskian form, i.e. $g_{i j} \stackrel{*}{=} \eta_{i j}$ and then (1.30) reduces to the form (1.4), in agreement with the equivalence principle.

Equation (1.30) can be written in terms of the Hodge dual operator * of to the metric $g$, namely

$$
H_{i j}=\sqrt{\frac{\varepsilon_{0}}{\mu_{0}}}\left({ }^{*} F\right)_{i j}, \quad\left({ }^{*} F\right)_{i j}:=\frac{1}{2} \hat{\epsilon}_{i j k l} \sqrt{|g|} g^{k m} g^{l n} F_{m n} .
$$

For the properties of these operator, see appendix C.3.

The Maxwell equations in a Riemannian space are therefore, in terms of $F$ and $g$ :

$$
\partial_{j}\left(\sqrt{\frac{\varepsilon_{0}}{\mu_{0}}} \sqrt{|g|} g^{i k} g^{j l} F_{k l}\right)=\mathcal{J}^{i}, \quad \epsilon^{i j k l} \partial_{j} F_{k l}=0 .
$$

The fact that the Maxwell equations (1.24) and (1.25) are naturally invariant and that the metric structure of space does not enter in their formulation is not an accident. It is a consequence of the fundamental property that the basic structure of electrodynamics can be derived from counting procedures of charge and magnetic flux, with do not require a metric (nor an affine) structure of spacetime. This properties will be further clarified in the axiomatic approach of chapter 2 . 


\section{Chapter 2}

\section{Electrodynamics on an arbitrary 4D-manifold}

In this chapter, we would like to present an axiomatic construction of classical electrodynamics which intends to be as general as possible. Structures are only introduced when they are indispensable for the development and not earlier than necessary. This approach will provide us a very general framework which can then be applied to many different particular cases.

We model spacetime as a smooth 4-dimensional manifold $X$ which, at least in some neighborhood, admits a foliation into 3-dimensional submanifolds, parameterized by a monotonic 'time' parameter $\sigma$, see figure 2.1.

\subsection{Charge conservation}

Probably the most important and defining property of electromagnetic theory is the experimentally well tested fact of charge conservation ${ }^{1}$. A basic property of electric charge is that it is an additive quantity which can be distributed in space, i.e. an extensive quantity. In nature, electric charge is know to be quantized, its fundamental quanta, the electric charges of the quarks are $\pm e / 3$ and $\pm 2 e / 3$, where $e$ denotes the electron charge. In a classical field theory, we describe the distribution of an extensive quantity in terms of a current density, a 3-form, containing the information of how many charges are distributed in spacetime, and how they move.

\footnotetext{
${ }^{1}$ See, for instance, $[59,85]$ for experiments testing charge conservation/violation.
} 


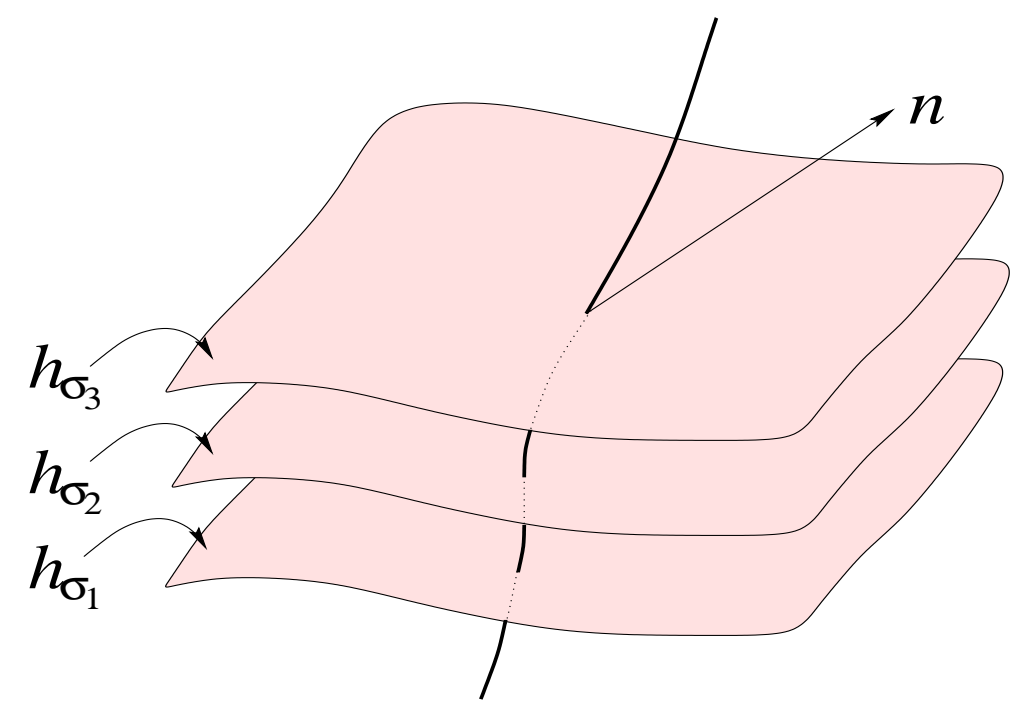

Figure 2.1: Foliation of spacetime: Each hypersurface $h_{\sigma}$ represents, at a 'time' $\sigma$, a 3-dimensional submanifold.

Consider the total charge within some 3 -dimensional region. This total charge should be independent of the orientation of any coordinate system used. Then, the electric charge distribution is described by the twisted electric current 3-form $J$, see appendix C.3 and [18] for definitions. For a 3dimensional region $\Sigma_{3}$ of a hypersurface $h_{\sigma}$ one can interpret $\int_{\Sigma_{3}} J$ as the total charge contained in $\Sigma_{3}$. On the other hand, if the 3-dimensional region is of the type $\Omega_{3}=\Sigma_{2} \times\left[\sigma_{1}, \sigma_{2}\right]$ one can interpret $\int_{\Omega_{3}} J$ as the total charge crossing the 2-dimensional surface $\Sigma_{2}$ during the 'time' interval $\left[\sigma_{1}, \sigma_{2}\right]$. Consequently, the 3 -form $J$ carries the dimension of charge, i.e. $[J]=q$.

Note that no concept of distance or parallel displacement, i.e. no metric or connection, are necessary to define the concepts of charge and charge current 3 -form specifically. Of course, quantities like 'charge per unit volume' and 'charge per unit area and unit time' are useful, after one provides prescriptions for what 'unit volume', 'unit area' and 'unit time' are. The latter are however concepts not needed to describe, e.g., how many electrons, and therefore how much charge, are contained in a certain region. Clearly, the total charge is independent of the unit in which volume is measured.

The components $J_{\alpha \beta \gamma}$ of the electric current 3-form with respect to some 


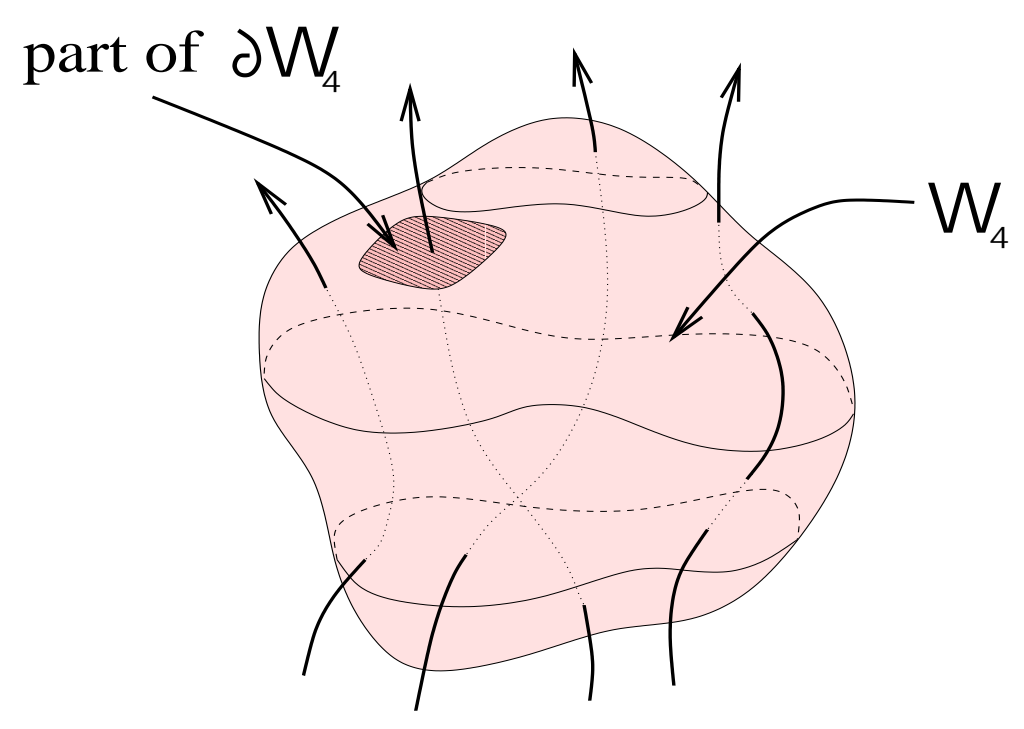

Figure 2.2: Local conservation of charge: Each worldline of a charged particle that enters the finite 4 -volume $\Omega_{4}$ via its boundary $\partial \Omega_{4}$ has also to leave $\Omega_{4}$.

coframe basis $\vartheta^{\alpha}, \alpha, \beta, \ldots=0,1,2,3$, are given by

$$
J=\frac{1}{3 !} J_{\alpha \beta \gamma} \vartheta^{\alpha} \wedge \vartheta^{\beta} \wedge \vartheta^{\gamma} .
$$

If one associates a dimension $l$, in the sense of a segment ${ }^{2}$ to the coframe $\vartheta$, i.e. $[\vartheta]=l$, then the components $J_{\alpha \beta \gamma}$ carry the dimension $\left[J_{\alpha \beta \gamma}\right]=q l^{-3}$.

The conservation of electric charge is then expressed as the vanishing of the integral

$$
\oint_{\partial \Omega_{4}} J=0, \quad \forall \Omega_{4},
$$

i.e., for any 3-dimensional boundary of a 4 -dimensional region $\Omega_{4}$. In particular, for a region $\Omega_{4}=\Sigma_{3} \times\left[\sigma_{1}, \sigma_{2}\right]$, the integral conservation law (2.2) requires the balance between the charge change in the region $\Sigma_{3}$ during the interval $\left[\sigma_{1}, \sigma_{2}\right]$ and the flux across its 2-dimensional boundary $\partial \Sigma_{3}$.

Since the region $\Omega_{4}$ in (2.2) is arbitrary, the Stokes theorem tells us that the current 3 -form must be closed, i.e.

$$
d J=0 .
$$

\footnotetext{
${ }^{2}$ i.e. a one dimensional extension on the manifold, not in the sense of a unit of length.
} 
Now, according to the de Rham theorem, see for instance [18], the current 3 -form is not only closed but also exact, i.e. it can be derived from some 2form $H$ by exterior derivation, if all its integrals over 3-dimensional regions without boundaries vanish. Under these assumptions, this means that there must be a 2 -form $H$ such that

$$
d H=J .
$$

The twisted 2-form $H$ is called the electromagnetic excitation, and must then carry dimension of charge, $[H]=q$. As we will see, in a $3+1$ decomposition its components can be identified with the usual dielectric displacement and magnetic field, see (1.21) and (1.22).

However, the conditions above are not enough to uniquely define the 2form $H$, since a 'gauge' transformation

$$
H \rightarrow H^{\prime}:=H+d \Psi
$$

leaves (2.4) invariant, for an arbitrary twisted 1-form $\Psi$.

Equation (2.4) are thus the inhomogeneous Maxwell equations (4 equations). More than defining the values of $H$, the above arguments show that the inhomogeneous Maxwell equations must be of the form (2.4), since this is the only kind of field equation which are compatible with electric charge conservation.

A single electromagnetic excitation will be picked out by the requirement that $H=0$ for $F=0$ for the spacetime/constitutive relation, see sections 2.5 and 2.6.

\subsection{Lorentz force}

We assume now that the concept of force density is known from mechanics and use it to define the electromagnetic field strength 2-form $F$, as usual, as force per unit charge. We define $F$ by means of

$$
\left.f_{\alpha}=:\left(e_{\alpha}\right\rfloor F\right) \wedge J \text {, }
$$

where $e_{\alpha}$ is a frame ${ }^{3}$ and $f_{\alpha} \in \Lambda^{4}(X)$ are the corresponding components of the force density covector-valued 4 -form in that frame ${ }^{4}$. From the definition

\footnotetext{
${ }^{3}$ This is again a metric-independent quantity. A frame is just a basis of the tangent space.

${ }^{4}$ Remember, in classical mechanics $f_{i}=\frac{\partial \mathcal{L}}{\partial x^{i}}$ (force), $p_{i}=\frac{\partial \mathcal{L}}{\partial \dot{x}^{i}}$ (momentum), and $f_{i}=\dot{p}_{i}$.
} 
(2.6) $F$ is a untwisted 2-form, i.e. an intensive quantity. The dimension of $F$ is $[F]=h / q$, with $h$ denoting the dimension of an action. The definition above is a very restrictive one, since it tells us that the force on test charges is determined only by the six independent components of the 2-form $F$, instead of the 16 independent quantities that a linear relation between force density and current density would in principle admit ${ }^{5}$. This assumption is part of our axiomatic, and it is suggested by the fact that the excitation is described by a field with 6 independent components and one therefore expects the field strength to have the same number of independent components. We know, of course, that this choice is reasonable since we know that in Maxwell's theory, the Lorentz force is determined by the six independent components of the electric and magnetic fields.

It is true that in order to have a complete predictive theory, one still has to specify the relation between velocities and momenta of test currents. This is required in order to be able to predict the evolution of test currents in a given electromagnetic field. This relation includes the metric tensor in the known case of GR. However, we are interested here in the structure of the general electromagnetic theory, and not in particular in this mechanical 'constitutive relation'. We assume that it is known, whether involving a metric tensor or not, so that the theory is complete.

\subsection{Magnetic flux conservation}

The next step in our axiomatic construction is to find conditions for the electromagnetic field strength to satisfy. The natural operation that can be done with a 2 -form is to integrate it on a given 2-dimensional region. If we assume, in analogy to conservation of charge, that

$$
\oint_{\partial \Omega_{3}} F=0,
$$

for an arbitrary 3-dimensional submanifold $\Omega_{3}$, then Stokes's theorem provides us with a differential equation for $F$, namely that the field strength must be closed,

$$
d F=0,
$$

\footnotetext{
${ }^{5}$ Consider for instance a relation of the form $f_{\alpha}=\Psi_{\alpha} \wedge J$. Then $\Psi_{\alpha}$ is a covector valued 1 -form, and has therefore 16 independent components.
} 
and (at least in some neighborhood of each event) exact, i.e. $F=d A$. The untwisted 1-form $A$ is then the electromagnetic potential.

We take (2.7), or equivalently (2.8) as third axiom. It represents the homogeneous Maxwell equations (4 equations) and expresses the conservation of magnetic flux. In general, magnetic flux is not quantized, as it is the case of electric charge. In Type II superconductors, however, as, e.g., in Niobium, quantized magnetic flux lines are possible.

This formulation does not admit magnetic charges (i.e. magnetic monopoles) in a natural way. Since the field strength $F$ is by its very definition an intensive quantity, a hypothetical magnetic charge density $\rho_{\mathrm{m}}$, such that $d F=\rho_{\mathrm{m}}$, would necessarily also be an intensive quantity (an untwisted 3form), quite in contrast to the extensive nature of any charge-like quantity like, e.g., electric charge, energy-momentum, all twisted 3-forms.

Locally at least, see above, the homogeneous Maxwell equation (2.8) imply that the field strength $F$ can be derived from a untwisted 1-form $A$, the electromagnetic potential, such that

$$
F=d A \text {. }
$$

Of course, $A$ is only determined up to a gauge transformation

$$
A \rightarrow A^{\prime}=A+d \Psi
$$

for any untwisted 0 -form (scalar) $\Psi$.

The electromagnetic potential carries the same physical dimension as the electromagnetic field strength, namely $[A]=h / q$.

\section{4 'Space'-'time' Decomposition}

Consider a foliation characterized by the monotonic 'time-like' parameter $\sigma$. Consider also a vector field $n$ not lying on a $\sigma=$ const. surface, i.e. $n\rfloor d \sigma \neq 0$, so that it can be used to 'evolve' the folia. See figure 2.1. In particular, one can rescale the vector field to make it fulfill the normalization condition $n\rfloor d \sigma=1$, which turns out to be useful.

We will call any $p$-form $\omega$ 'transverse with respect to the vector $n$ ' if $n\rfloor \omega=0$. Then, given any $p$-form $\Psi$ one can define the $p$-form $\Psi$ and the

$(p-1)$-form $\Psi_{\perp}$, both of which are transverse with respect to $n$ in the above sense, as

$$
\underline{\Psi}:=n\rfloor(d \sigma \wedge \Psi),
$$




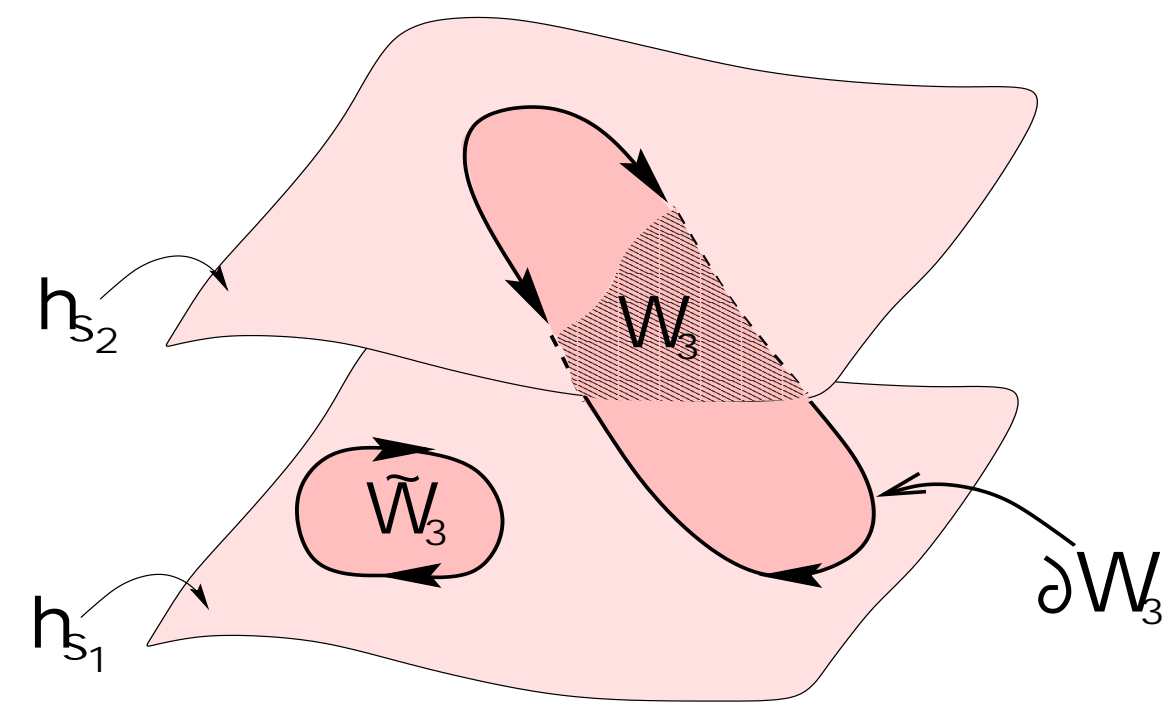

Figure 2.3: Conservation of magnetic flux in spacetime: For an arbitrary 3-dimensional integration domain $\Omega_{3}$, the integral $\underset{\partial \Omega_{3}}{\oint_{3}} F$ vanishes.

and

$$
\left.\Psi_{\perp}:=n\right\rfloor \Psi
$$

For $p=n$ we have $\underline{\Psi}=0$. Similarly, for $p=0, \Psi_{\perp}=0$.

These two quantities $\underline{\Psi}$ and $\Psi_{\perp}$ contain the complete information of the original form $\Psi$. The later can be written as

$$
\Psi=d \sigma \wedge \Psi_{\perp}+\underline{\Psi} .
$$

From their definition, $\underline{\Psi}$ and $\Psi_{\perp}$ satisfy the following properties

- Transversality of $\Psi_{\perp}$ with respect to $n$,

$$
n\rfloor \Psi_{\perp}=0
$$

- $\perp$ of a product,

$$
(\Psi \wedge \Phi)_{\perp}=\Psi_{\perp} \wedge \underline{\Phi}+(-1)^{p} \underline{\Psi} \wedge \Phi_{\perp}
$$


- _ of a product,

$$
\underline{\Psi \wedge}=\underline{\Psi} \wedge \underline{\Phi}
$$

- Lie derivative $\mathcal{L}_{n}$ and ${ }_{\perp}$ commute,

$$
\left(\mathcal{L}_{n} \Psi\right)_{\perp}=\mathcal{L}_{n} \Psi_{\perp}
$$

- Lie derivative $\mathcal{L}_{n}$ and _ commute,

$$
\underline{\mathcal{L}_{n} \Psi}=\mathcal{L}_{n} \underline{\Psi}
$$

Additionally, it is useful to define a transverse part of the exterior derivative, $\underline{d}$, such that for any $p$-form $\Psi$

$$
\underline{d} \Psi:=n\rfloor(d \sigma \wedge \Psi) .
$$

It has the following properties:

- Transversality of $\underline{d}$ with respect to $n$,

$$
\underline{\underline{d}} \Psi=n\rfloor(\underline{d} \Psi) \equiv 0,
$$

- $\perp$ of a derivative,

$$
(d \Psi)_{\perp}=\mathcal{L}_{n} \underline{\Psi}-\underline{d} \Psi_{\perp},
$$

- _ of a derivative,

$$
\underline{d \Psi}=\underline{d} \underline{\Psi} .
$$

By means of this general decomposition procedure, we decompose the Maxwell equations (2.4) and (2.8). First decompose the fields $J, H, F$ and $A$ and introduce the notation

$$
\begin{array}{ll}
j:=-J_{\perp}, & \rho:=\underline{J} . \\
\mathcal{H}:=H_{\perp}, & \mathcal{D}:=\underline{H}, \\
E:=-F_{\perp}, & B:=\underline{F},
\end{array}
$$




$$
\varphi:=A_{\perp}, \quad \mathcal{A}:=\underline{A} .
$$

The 4-dimensional quantities can be reconstructed according to

$$
\begin{aligned}
& J=-j \wedge d \sigma+\rho, \\
& H=-\mathcal{H} \wedge d \sigma+\mathcal{D}, \\
& F=E \wedge d \sigma+B, \\
& A=\varphi \wedge d \sigma+\mathcal{A} .
\end{aligned}
$$

Now one can take the inhomogeneous Maxwell equation (2.4) and find

$$
\begin{aligned}
(d H-J)_{\perp} & =(d H)_{\perp}-J_{\perp} \\
& =\mathcal{L}_{n} \underline{H}-\underline{d} H_{\perp}-J_{\perp} \\
& =\mathcal{L}_{n} \mathcal{D}-\underline{d} \mathcal{H}+j, \\
\underline{d H-J} & =\underline{d H}-\underline{J} \\
& =\underline{d} \underline{H}-\underline{J} \\
& =\underline{d} \mathcal{D}-\rho .
\end{aligned}
$$

Analogously, from the homogeneous Maxwell equations (2.8) we obtain

$$
\begin{aligned}
(d F)_{\perp} & =\mathcal{L}_{n} \underline{F}-\underline{d} F_{\perp} \\
& =\mathcal{L}_{n} B+\underline{d} E, \\
\underline{d F} & =\underline{d} \underline{F} \\
& =\underline{d} B .
\end{aligned}
$$

Thus, we find the Maxwell equations in a $3+1$ decomposed form to be

$$
\begin{aligned}
\underline{d} \mathcal{D}=\rho, & \underline{d} \mathcal{H}=\mathcal{L}_{n} \mathcal{D}+j, \\
\underline{d} \mathcal{B}=0, & \underline{d} E+\mathcal{L}_{n} B=0 .
\end{aligned}
$$

We can now decompose the law of charge conservation (2.3). It is important to note that no information is obtained from $\underline{d J}$ since this quantity 
vanishes for any 3 -form $J$, as can be seen from the definition (2.11). We then compute

$$
\begin{aligned}
(d J)_{\perp} & =\mathcal{L}_{n} \underline{J}-\underline{d} J_{\perp} \\
& =\mathcal{L}_{n} \rho+\underline{d} j,
\end{aligned}
$$

so that charge conservation means

$$
\mathcal{L}_{n} \rho+\underline{d} j=0 .
$$

Finally, we decompose equation (2.9). We find

$$
\begin{aligned}
(d A)_{\perp} & =\mathcal{L}_{n} \underline{A}-\underline{d} A_{\perp} \\
& =\mathcal{L}_{n} \mathcal{A}-\underline{d} \varphi, \\
\underline{d A} & =\underline{d} \underline{A} \\
& =\underline{d} \mathcal{A},
\end{aligned}
$$

so that

$$
E=\underline{d} \varphi-\mathcal{L}_{n} \mathcal{A}, \quad B=\underline{d} \mathcal{A} .
$$

The decomposed Maxwell equations (2.35) and (2.36) naturally generalize equations (1.11) and (1.12) from chapter 1.4. The Lie derivative $\mathcal{L}_{n}$ is the natural generalization of the time derivative since it measures the change of the (integral of the) corresponding field between folia, according to the displacement induces by the vector field $n$, see figure 2.4.

\subsection{Measuring $H$}

In this section, we discuss a general procedure for measuring the excitation $H$. This can be done by using an idealized object, namely, and ideal conductor. This special material is assumed to have the following two properties:

1. In an ideal conductor, all charges are located on its surface. In other words, the ideal conductor is such that inside it no free charges can be found. If $\Omega_{3}$ is the 3 -dimensional region describing the conductor, then the free charges are all located on $\partial \Omega_{3}$. 


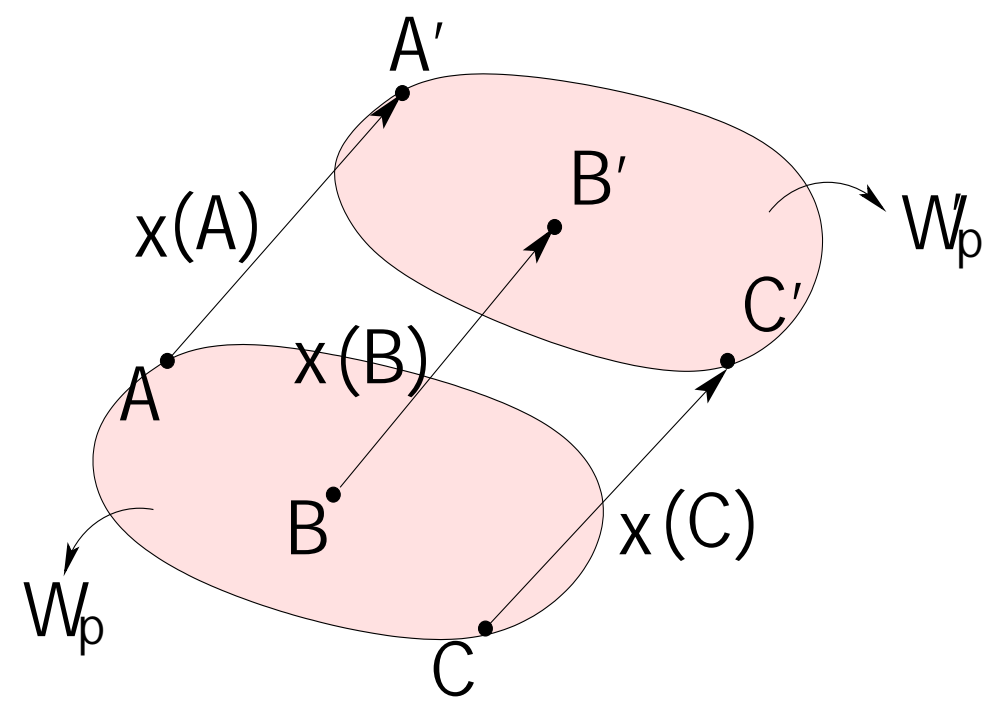

Figure 2.4: Under the displacement $x^{i} \rightarrow x^{i}=x^{i}+a n^{i}(x)$ ( $a$ being an infinitesimal constant parameter) the integral $\int_{\Omega_{p}^{\prime}} \Psi$ of a $p$-form $\Psi$ over the corresponding $p$-dimensional mapped region $\Omega_{p}^{\prime}$ is given by $\int_{\Omega_{p}^{\prime}} \Psi=\int_{\Omega_{p}} F+$ $a \int_{\Omega_{p}} \mathcal{L}_{n} \Psi$.

2. In the 'rest frame' of the conductor the electric excitation $\mathcal{D}$ vanishes. If at some event inside the conductor a volume element is spanned by the vectors $e_{a}, a, b, \ldots=1,2,3$ and $e_{0}=n$ is a vector pointing in the fourth independent 'time' direction, then we assume $\left.\left.H_{a b}:=e_{b}\right\rfloor e_{a}\right\rfloor H=0$, or equivalently $H=d \sigma \wedge \mathcal{H}$, with $n\rfloor d \sigma=1$ and $\left.e_{a}\right\rfloor d \sigma=0$, see (2.28).

With these assumptions one can measure the excitation by use of 'Maxwellian double plates'. Consider two (uncharged) parallel plates made of an ideal conductor and locate them at the point $P$ where the excitation should be measured. The field strength (whatever value it may have) will induce surface charges in the conductor. Separate now the plates and measure the charge $Q$ induced in one of its surfaces. One can then integrate the inhomogeneous Maxwell equation over a volume with one side in one conducting plate and the other between the plates, see figure 2.5. In the limit of vanishing 'thickness' of $\Omega_{3}$, see fig. 2.5 , one finds

$$
\left.\left.\left.\left.\int_{\Omega_{3}} d H=\int_{\partial \Omega_{3}} H=\left(e_{2}\right\rfloor e_{1}\right\rfloor H\right)_{\mathrm{P}}-\left(e_{2}\right\rfloor e_{1}\right\rfloor H\right)_{\text {cond }}
$$



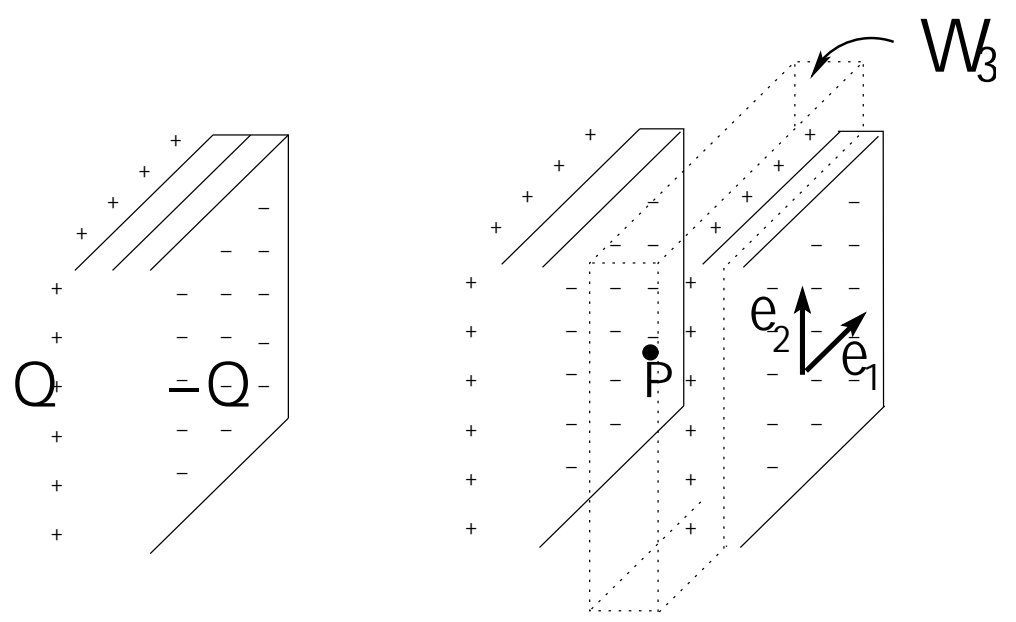

Figure 2.5: Maxwellian plates. Here $e_{1}$ and $e_{2}$ span the surface element parallel to the plates.

and $\int_{\Omega_{3}} J=Q$, so that

$$
\left(H_{12}\right)_{\mathrm{P}}-\left(H_{12}\right)_{\text {cond }}=Q \text {. }
$$

The second term on the left hand side of (2.43) vanishes because of the property 2 of ideal conductors. Therefore, the induced charge $Q$ determines the component $H_{12}$ of the excitation. Similarly, by orienting the plates differently one can measure, e.g. $H_{13}$. Furthermore, by changing the state of motion of the conductor (i.e. different 4-velocities) one can measure components of $H$ which are, in the notation we are using, of the form $\left.\left.\left(e_{1}\right\rfloor e_{0}\right\rfloor H\right)=H_{01}$.

\subsection{Constitutive relations}

As it is clear from the last sections, the Maxwell equations in their form (2.4) and (2.8) are valid for any medium. They describe the general features of electrodynamics which follow from charge and magnetic flux conservation. In this form, Maxwell equations are valid, for instance, in vacuum in Special Relativity, but also if gravitational effects are included in the context of General Relativity or alternative theories, as for instance in those formulated in a general metric-affine spacetime. They are also applicable to any material 


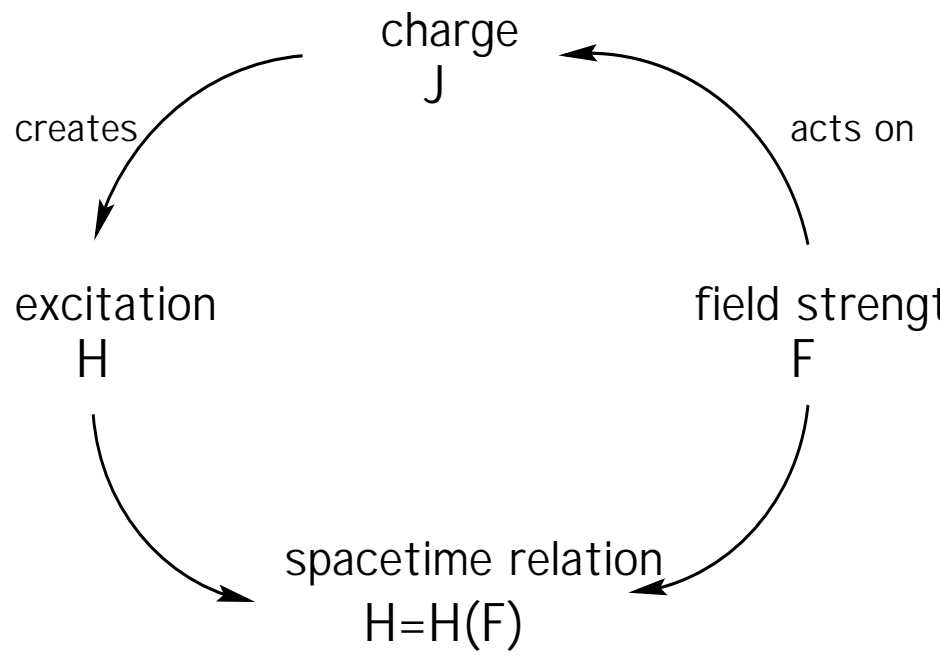

Figure 2.6: The spacetime/constitutive relation connecting excitation and field strength.

medium. In other words, (2.4) and (2.8) are general structures of electromagnetic theory. The additional structure that really defines the particular physical properties of the system under consideration, is the subject of this section.

We saw that the Maxwell equations (2.4) and (2.8) amount to only 8 independent equations for the 12 independent fields, $H$ and $F$. A further relation between the excitation and field strength, i.e. a connection between the field generated by the charges and the field acting on test currents, is therefore necessary in order to make the theory complete. Such a relation is referred to as the constitutive relation when corresponding to some material medium, and as the spacetime relation when describing the vacuum properties. The formalism presented here can thus be used in both situations. In the first one, the current 3-form describes the so-called 'external' ('free') currents flowing through a material medium. In vacuum, $J$ represents the total current density. In any case, the relation between $H$ and $F$ describes the properties of the arena in which the electromagnetic phenomena of interest take place.

Actually, under some circumstances, the distinction can even be diffuse. This is related to the problem of deciding whether the charge current $J$, which is the starting point of the formalism, corresponds to the 'fundamen- 
tal charges' of the system or if they are 'total' charges within a given region. This decision is in general part of the physical assumptions of the particular electromagnetic model under consideration. For instance, in a material medium one can consider the charge current $J$ as the free, unbound charge and base the corresponding electromagnetic theory on this assumption. We know, however, that this medium has an microscopic structure, since the material is composed by atoms, which are formed by protons, neutrons and electrons, with positive, zero and negative electric charges respectively. Then one can construct a more fundamental electromagnetic theory when $J$ now resolves the individual proton and electron charges, and the constitutive law corresponds to that of 'vacuum', i.e. to what we call the spacetime relation. One could however go a step further and recognize the fact that the classical vacuum can be understood, according Quantum Electrodynamics (QED), as a 'medium' in which particle-antiparticle pairs are continuously created and destroyed. Macroscopically, however, only the total charge of, say, electrons and protons survive. The Heisenberg-Euler spacetime relation is an explicit example of this approach, which includes the effects of quantum corrections to Maxwell-Lorentz electrodynamics. On the other hand, the above arguments mean that one could in principle construct an electromagnetic theory in which the classical vacuum is now treated as a composite system. This kind of approach is not usually followed, but shows that the identification of what is understood as being 'vacuum' depends on the model for the charges of the system.

In general, constitutive relations $H=H(F)$ can have any functional dependence, i.e, they can be non-linear and non-local. Non-local constitutive relations are common for material media because in general the macroscopic electromagnetic properties at some point of the material are influenced by the field and charge configuration at other points of it. Additionally, the finite propagation speed of the microscopical electromagnetic interaction between the different parts of the material leads to time-like non-locality. In many cases (typically, for slowly varying fields), however, this non-local effects are negligible and the medium can be treated as if the field excitation $H$ where depending only of the value of the field strength at the same point, i.e. with a local constitutive law. In what follows, we restrict ourselves only to this later case. 


\subsubsection{Linear constitutive relations}

We now concentrate on the particular case in which the field strength and excitations are proportional. A great number of material media are known in which this is valid for a variety of conditions, see for instance [45]. We also expect the spacetime relation (i.e. the vacuum relation) to be simple and, in particular, linear. Linearity is taken for granted in all traditional approaches to vacuum electrodynamics.

Given a local frame $\vartheta^{\alpha}$, with $\alpha=\hat{0}, \hat{1}, \hat{2}, \hat{3}$, we can decompose the exterior forms $H$ and $F$ as

$$
H=\frac{1}{2} H_{\alpha \beta} \vartheta^{\alpha} \wedge \vartheta^{\beta}, \quad F=\frac{1}{2} F_{\alpha \beta} \vartheta^{\alpha} \wedge \vartheta^{\beta},
$$

and write a general linear constitutive law (spacetime relation) as

$$
H_{\alpha \beta}=\frac{1}{2} \kappa_{\alpha \beta}^{\gamma \delta} F_{\gamma \delta}=\frac{1}{4} \hat{\epsilon}_{\alpha \beta \gamma \delta} \chi^{\gamma \delta \epsilon \theta} F_{\epsilon \theta},
$$

where $\hat{\epsilon}_{\alpha \beta \gamma \delta}$ is the Levi-Civita symbol with $\hat{\epsilon}_{\hat{0} \hat{1} \hat{2} \hat{3}}:=1$ and $\chi^{\alpha \beta \gamma \delta}$ is called the constitutive tensor density, which is an untwisted tensor density of weight +1 , carrying dimension $[\chi]=[H] /[F]=q^{2} / h$.

From its definition, the constitutive tensor satisfies the following symmetry properties

$$
\chi^{\alpha \beta \gamma \delta}=-\chi^{\beta \alpha \gamma \delta}=-\chi^{\alpha \beta \delta \gamma},
$$

which means that it has 36 independent components. Due to these symmetry properties the constitutive tensor can also be represented by $6 \times 6$ matrix, say $\chi^{I J}$, where each index $I, J, \ldots$ corresponds to a pair of antisymmetrized 4-dimensional indices, i.e., $I \rightarrow\left[i_{1} i_{2}\right]$, etc. In this notation, e.g., $\epsilon^{I J} \rightarrow$ $\epsilon^{i_{1} i_{2} j_{1} j_{2}}$. One can enumerate the 6 -dimensional indices according to $I, J, \ldots=$ $01,02,03,23,31,12$.

We want to identify some irreducible components of the constitutive tensor. Remember that so far no metric structure has been introduced. However, we always have the Levi-Civita tensor density $\epsilon^{\alpha \beta \gamma \delta}$ at our disposal, and thus we can decompose the constitutive tensor according to, see [76],

$$
\chi^{\alpha \beta \gamma \delta}={ }^{(1)} \chi^{\alpha \beta \gamma \delta}+{ }^{(2)} \chi^{\alpha \beta \gamma \delta}+{ }^{(3)} \chi^{\alpha \beta \gamma \delta},
$$

where the different irreducible pieces ${ }^{(1)} \chi,{ }^{(2)} \chi$, and ${ }^{(3)} \chi$ are determined by the symmetry properties

$$
{ }^{(1)} \chi^{\alpha \beta \gamma \delta}={ }^{(1)} \chi^{\gamma \delta \alpha \beta}, \quad{ }^{(1)} \chi^{[\alpha \beta \gamma \delta]}=0,
$$




$$
\begin{aligned}
& { }^{(2)} \chi^{\alpha \beta \gamma \delta}=-{ }^{(2)} \chi^{\gamma \delta \alpha \beta}, \\
& { }^{(3)} \chi^{\alpha \beta \gamma \delta}={ }^{(3)} \chi^{[\alpha \beta \gamma \delta]},
\end{aligned}
$$

or, explicitly,

$$
\begin{aligned}
{ }^{(3)} \chi^{\alpha \beta \gamma \delta}:=\chi^{[\alpha \beta \gamma \delta]}, \\
{ }^{(2)} \chi^{\alpha \beta \gamma \delta}:=\frac{1}{2}\left[\chi^{\gamma \delta \alpha \beta}-\chi^{\alpha \beta \gamma \delta}\right], \\
{ }^{(1)} \chi^{\alpha \beta \gamma \delta}:=\chi^{\alpha \beta \gamma \delta}-{ }^{(2)} \chi^{\alpha \beta \gamma \delta}-{ }^{(3)} \chi^{[\alpha \beta \gamma \delta]} .
\end{aligned}
$$

One can introduce an alternative, but equivalent, parametrization of the 15 independent components of the asymmetric piece ${ }^{(2)} \chi$ in terms of a traceless second rank tensor $S_{\alpha}^{\beta}$ (thus, also with 15 independent components) [32] as

$$
{ }^{(2)} \chi^{\alpha \beta \gamma \delta}=\epsilon^{\alpha \beta \epsilon[\gamma} S_{\epsilon}{ }^{\delta]}-\epsilon^{\gamma \delta \epsilon[\alpha} S_{\epsilon}{ }^{\beta]}, \quad S_{\alpha}{ }^{\alpha}=0 .
$$

Now, contracting (2.54) with the Levi-Civita symbol, one finds that

$$
S_{\alpha}^{\beta}=\frac{1}{4} \hat{\epsilon}_{\alpha \gamma \delta \epsilon}{ }^{(2)} \chi^{\gamma \delta \epsilon \beta}
$$

which shows that the traceless tensor $S_{\alpha}{ }^{\beta}$ is uniquely determined by (2.54).

The third piece ${ }^{(3)} \chi$ can always be written as

$$
{ }^{(3)} \chi^{\alpha \beta \gamma \delta}=\alpha(x) \epsilon^{\alpha \beta \gamma \delta}
$$

where $\alpha(x)$ is uniquely determined to be

$$
\alpha:=\frac{1}{4 !} \hat{\epsilon}_{\alpha \beta \gamma \delta} \chi^{\alpha \beta \gamma \delta}
$$

It is called an abelian axion field.

Denote the contribution of each piece of the constitutive tensor to the excitation as ${ }^{(1)} H,{ }^{(2)} H$ and ${ }^{(3)} H$, such that

$$
{ }^{(1)} H_{\alpha \beta}=\frac{1}{4} \hat{\epsilon}_{\alpha \beta \gamma \delta}{ }^{(1)} \chi^{\gamma \delta \epsilon \theta} F_{\epsilon \theta},
$$

and analogously for the other pieces. Then

$$
H={ }^{(1)} H+{ }^{(2)} H+{ }^{(3)} H,
$$


and

${ }^{(3)} H=\alpha F$.

Using (2.4) and (2.8), we find

$$
\begin{aligned}
d H & =d\left({ }^{(1)} H+{ }^{(2)} H+{ }^{(3)} H\right) \\
& =d\left({ }^{(1)} H+{ }^{(2)} H\right)+d(\alpha \wedge F) \\
& =d\left({ }^{(1)} H+{ }^{(2)} H\right)+d \alpha \wedge F,
\end{aligned}
$$

showing that the axion piece contributes to the Maxwell equations only if $d \alpha \neq 0$.

Equation (2.47) represents the irreducible decomposition of the corresponding $6 \times 6$ matrix with respect to the linear group into a symmetric traceless piece, an antisymmetric piece and a trace piece. The Levi-Civita symbol serves as a kind of 'metric' in the 6-dimensional formulation $\left(\epsilon^{i j k l} \rightarrow \epsilon^{I J}\right)$ which is used to construct the traces of $\chi^{I J}$. The constitutive tensor is therefore reduced as $\chi={ }^{(1)} \chi+{ }^{(2)} \chi+{ }^{(3)} \chi$ in $36=20+15+1$ independent components, respectively. No further decomposition of the constitutive tensor is possible at this point, since no additional geometric objects are available.

For later application, see section 3.1, we abbreviate the action of the irreducible pieces ${ }^{(1)} \chi$ and ${ }^{(2)} \chi$ by defining the operator ${ }^{\#}: \Lambda^{2} \rightarrow \Lambda^{2}$ such that

$$
{ }^{\#} \Omega:=\frac{1}{4} \hat{\epsilon}_{\alpha \beta \gamma \delta}\left({ }^{(1)} \chi^{\gamma \delta \epsilon \theta}+{ }^{(2)} \chi^{\alpha \beta \gamma \delta}\right) \Omega_{\epsilon \theta} \vartheta^{\alpha} \wedge \vartheta^{\beta}
$$

for any 2 -form $\Omega$ with frame components $\Omega_{\alpha \beta}$. Then we can rewrite our constitutive/spacetime relation $(2.45)$ as

$$
H={ }^{\#} F+\alpha(x) F .
$$

Each irreducible piece is expected to describe different aspects of the medium. Additional information about the different properties of each piece will be obtained with the study of the electromagnetic energy-momentum current and of wave propagation. Notice for example that, if ${ }^{(2)} \chi \neq 0$ the Maxwell equations cannot follow as Euler-Lagrange equations from a Lagrangian of the form $V:=H \wedge F$, since ${ }^{(2)} \chi \neq 0$ drops out from $V$ due to its symmetry properties. However, our intention is to try to develop our electromagnetic theory as generally as possible. Therefore we want to include 
systems for which no Lagrange density can be found. Typical examples of such kind of physical systems are those including dissipative effects. One can therefore expect the 'extra' irreducible piece ${ }^{(2)} \chi$ to be related to some kind of intrinsic dissipative property of the medium. As we will see in section 2.7, this is indeed the case. Furthermore, constitutive laws (for matter) with ${ }^{(2)} \chi \neq 0$ (non-vanishing "skewon fields") have been discussed by Nieves and Pal $[57,58]$. They yield $T$ - and $P$-violating terms in the field equations.

Non-abelian axions were postulated for the first time by Peccei and Quinn [66], see also [104, 106]. Abelian axions coupling to electromagnetism were first considered by $\mathrm{Ni}[55]$ and correspond to a non-vanishing piece ${ }^{(3)} \chi$. There have been intensive experimental searches for axions, see $[56,84,96]$ and references therein. To date, no evidence of such a field has been found. Constraints on the axion mass and coupling to the electromagnetic field have been obtained both from astrophysical observations as from laboratory experiments, see $[56,84,96]$ for details. However, as we will see later, the axion-like term does not enter into important quantities as the energy-momentum current and the Fresnel equation. The axion remains a serious candidate for a particle search in experimental high energy physics and is a candidate for cold dark matter. The discussion about the possible existence of such field for some material medium has been rather controversial, see [102, 103, 93] and references therein. In this context the vanishing on the axion piece is referred to as the 'Post constraint' (PC), after the work of Post [72]. Sihvola and collaborators have correctly recognized that the axion piece is allowed by the basic structure of electrodynamics. However, if the medium is homogeneous, $\alpha$ is constant ${ }^{6}$ and then the corresponding term drops out completely from the Maxwell field equations. Therefore, a possible axion field can only be detected if it is inhomogeneous $(d \alpha \neq 0)$, or eventually by its effects on the boundary separating two homogeneous media with different axion fields each. Some theoretical work on the reflexion and transmition properties of this kind of medium can be found in [93] and references therein. No clear example of a material medium with a nontrivial axion-like term in its constitutive law has been reported in the literature.

Finally, some astrophysical observations have been used to constrain some components of a possible constitutive tensor deviating from the vacuum minkowskian one, see $[24,17,41]$.

\footnotetext{
${ }^{6}$ by the very definition of an homogenous medium.
} 


\section{Three dimensional decomposition of the constitutive tensor}

In some particular applications in which a $3+1$ decomposition is used, as for instance in nonrelativistic and/or noncovariant formulations of electrodynamics, see section 2.6.1 for a particular example, it is convenient to express the constitutive tensor (36 components) in terms of four $3 \times 3$ matrices $\mathcal{A}, \mathcal{B}, \mathcal{C}$ and $\mathcal{D}$ (each with 9 independent components). We define ${ }^{7}$ them as follows:

$$
\begin{aligned}
& \mathcal{A}^{b a}:=\chi^{0 a 0 b}, \quad \mathcal{B}_{b a}:=\frac{1}{4} \hat{\epsilon}_{a c d} \chi^{c d e f} \hat{\epsilon}_{e f b}, \\
& \mathcal{C}^{b}{ }_{a}:=\frac{1}{2} \hat{\epsilon}_{a c d} \chi^{c d 0 b}, \quad \mathcal{D}_{b}^{a}:=\frac{1}{2} \chi^{0 a c d} \hat{\epsilon}_{c d b},
\end{aligned}
$$

Here $a, b, c, \ldots=1,2,3$. The inverse relations are

$$
\begin{aligned}
& \chi^{0 a 0 b}=\mathcal{A}^{b a}, \quad \chi^{0 a b c}=\mathcal{D}_{d}^{a} \epsilon^{d b c}, \\
& \chi^{a b 0 c}=\epsilon^{a b d} \mathcal{C}_{d}^{c}, \quad \chi^{a b c d}=\epsilon^{a b e} \mathcal{B}_{f e} \epsilon^{f c d},
\end{aligned}
$$

Additionally, one can $(3+1)$-decompose the coordinate components of field excitation and strength as

$$
\begin{aligned}
\mathcal{D}^{a} & :=\left(H_{23}, H_{31}, H_{12}\right), \quad \mathcal{H}_{a}:=\left(H_{01}, H_{02}, H_{03}\right), \\
B^{a} & :=\left(F_{23}, F_{31}, F_{12}\right), \quad E_{a}:=\left(F_{10}, F_{20}, F_{30}\right) .
\end{aligned}
$$

With these definitions, the spacetime/constitutive relation can be written as

$$
\mathcal{D}^{a}:=-\mathcal{A}^{b a} E_{b}+\mathcal{D}_{b}{ }^{a} B^{b}, \quad \mathcal{H}_{a}:=-\mathcal{C}^{b}{ }_{a} E_{b}+\mathcal{B}_{b a} B^{b}
$$

Furthermore, using the 6-dimensional notation, we have

$$
H_{I}=\left(\mathcal{H}_{a}, \mathcal{D}^{a}\right), \quad F_{I}=\left(-E_{a}, B^{a}\right) .
$$

And the spacetime relation is written as

$$
H_{I}=\epsilon_{I J} \chi^{J K} F_{K}
$$

\footnotetext{
${ }^{7}$ The conventions in these definitions are taken such that they are consistent with those of [31].
} 
with

$$
\chi^{I J}=\left(\begin{array}{cc}
\mathcal{B}_{a b} & \mathcal{D}_{a}{ }^{b} \\
\mathcal{C}^{a}{ }_{b} & \mathcal{A}^{a b}
\end{array}\right), \quad \epsilon^{I J}=\epsilon_{I J}=\left(\begin{array}{cc}
0_{3} & 1_{3} \\
1_{3} & 0_{3}
\end{array}\right) .
$$

Then the irreducible decomposition of $\chi$ can be found to be

$$
\begin{aligned}
& { }^{(1)} \chi^{I J}=\left(\begin{array}{cc}
\mathcal{B}_{(a b)} & \frac{1}{2}\left(\mathcal{D}_{a}{ }^{b}+\mathcal{\ell}^{b}{ }_{a}\right) \\
\frac{1}{2}\left(\mathcal{C}^{a}{ }_{b}+\mathcal{D}_{b}{ }^{a}\right) & \mathcal{A}^{(a b)}
\end{array}\right), \\
& { }^{(2)} \chi^{I J}=\left(\begin{array}{cc}
\mathcal{B}_{[a b]} & \frac{1}{2}\left(\mathcal{C}^{b}{ }_{a}-\mathcal{D}_{a}{ }^{b}\right) \\
\frac{1}{2}\left(\mathcal{D}_{b}{ }^{a}-\mathcal{C}^{a}{ }_{b}\right) & \mathcal{A}^{[a b]}
\end{array}\right), \\
& { }^{(3)} \chi^{I J}=\frac{1}{6}\left(\mathcal{C}^{c}{ }_{c}+\mathcal{D}_{c}{ }^{c}\right) \epsilon^{I J} .
\end{aligned}
$$

We introduced the 3-dimensional traceless quantities

$$
\mathcal{Q}_{b}^{a}:=\mathcal{C}_{b}^{a}-\frac{1}{3} \mathcal{C}_{c}^{c} \delta_{b}^{a}, \quad \not{D}_{a}^{b}:=\mathcal{D}_{a}^{b}-\frac{1}{3} \mathcal{D}_{c}{ }^{c} \delta_{a}^{b},
$$

so that

$$
\ell_{a}^{a}=0, \quad \not{D}_{a}^{a}=0 .
$$

In terms of $S$, see (2.55), the second irreducible piece of the 3-dimensional matrices (2.64)-(2.65) are found to be given by

$$
\begin{aligned}
& { }^{(2)} \mathcal{A}^{b a}=\epsilon^{a b c} S_{c}{ }^{0}, \quad{ }^{(2)} \mathcal{B}_{b a}=-\hat{\epsilon}_{a b c} S_{0}{ }^{c}, \\
& { }^{(2)} \mathcal{C}^{b}{ }_{a}=-S_{a}{ }^{b}+\delta_{a}^{b} S_{c}{ }^{c}, \quad{ }^{(2)} \mathcal{D}_{b}{ }^{a}=S_{b}{ }^{a}-\delta_{b}^{a} S_{c}{ }^{c} .
\end{aligned}
$$

Therefore

$$
{ }^{(2)} \chi^{I J}=\left(\begin{array}{cc}
{ }^{(2)} \mathcal{B}_{a b} & { }^{(2)} \mathcal{D}_{a}{ }^{b} \\
{ }^{(2)} \mathcal{C}^{a}{ }_{b} & { }^{(2)} \mathcal{A}^{a b}
\end{array}\right)=\left(\begin{array}{cc}
\hat{\epsilon}_{a b c} S_{0}{ }^{c} & +S_{a}{ }^{b}-\delta_{a}^{b} S_{c}{ }^{c} \\
-S_{b}{ }^{a}+\delta_{b}^{a} S_{c}{ }^{c} & -\epsilon^{a b c} S_{c}{ }^{0}
\end{array}\right)
$$

and

$$
\begin{aligned}
{ }^{(2)} \mathcal{D}^{a} & =\epsilon^{a b c} S_{b}{ }^{0} E_{c}+\left(S_{b}{ }^{a}-\delta_{b}^{a} S_{c}{ }^{c}\right) B^{b} \\
{ }^{(2)} \mathcal{H}_{a} & =\hat{\epsilon}_{a b c} S_{0}{ }^{b} B^{c}+\left(S_{a}{ }^{b}-\delta_{a}^{b} S_{c}{ }^{c}\right) E_{b} .
\end{aligned}
$$




\section{The asymmetric constitutive tensor of Nieves and Pal}

Consider the particular case in which $S_{i}{ }^{j}$ is the traceless part of the product of a covector $\omega$ and a vector $v$, i.e.,

$$
S_{i}^{j}=\omega_{i} v^{j}-\frac{1}{4}\left(\omega_{k} v^{k}\right) \delta_{i}^{j}
$$

Then we have

$$
\begin{aligned}
& { }^{(2)} \mathcal{A}^{b a}=v^{0} \epsilon^{a b c} \omega_{c}, \quad{ }^{(2)} \mathcal{B}_{b a}=-\omega_{0} \hat{\epsilon}_{a b c} v^{c}, \\
& { }^{(2)} \mathcal{C}^{b}{ }_{a}=-\left[\omega_{a} v^{b}+\frac{1}{2} \delta_{a}^{b}\left(\omega_{0} v^{0}-\omega_{c} v^{c}\right)\right], \\
& { }^{(2)} \mathcal{D}_{b}{ }^{a}=\omega_{b} v^{a}+\frac{1}{2} \delta_{b}^{a}\left(\omega_{0} v^{0}-\omega_{c} v^{c}\right) .
\end{aligned}
$$

As we mentioned, Nieves and Pal [57, 58] discussed P- and T-violating generalizations of the Maxwell-Lorentz equations for isotropic materia media. The constitutive tensor they study has a nonvanishing skew-symmetric piece ${ }^{(2)} \chi$. A 4-dimensional formulation of the constitutive tensor (Nieves and Pal used the $3+1$ form of Maxwell's equations in cartesian coordinates) can be given by considering, in addition to the Minkowski metric $\eta$, a time-like vector $v$, which explicitly breaks Lorentz invariance.

Consider, in addition to the usual 'metric' piece

$$
{ }^{(1)} \chi^{i j k l}=\sqrt{\frac{\varepsilon_{0}}{\mu_{0}}}\left(\eta^{i k} \eta^{j l}-\eta^{j k} \eta^{i l}\right)=2 \sqrt{\frac{\varepsilon_{0}}{\mu_{0}}} \eta^{i[k} \eta^{l] j},
$$

an antisymmetric piece ${ }^{(2)} \chi$ defined by (2.54) and (2.84) for the particular case in which $\omega$ and $v$ are, in cartesian coordinates $\omega_{a} \stackrel{*}{=}(\omega, 0,0,0)$ and $v^{a} \stackrel{*}{=}(v, 0,0,0)$. This choice implies in particular that the material will look spatially isotropic in this frame. The corresponding 3-dimensional constitutive matrices $\mathcal{A}^{a b}, \mathcal{B}_{a b}, \mathcal{C}^{a}{ }_{b}$ and $\mathcal{D}_{a}{ }^{b}$, according to their definitions (2.64) and (2.65), are then found to be

$$
\begin{aligned}
& \mathcal{A}^{a b} \stackrel{*}{=}-\varepsilon_{0} \delta^{a b}, \quad \mathcal{B}_{a b} \stackrel{*}{=} \mu_{0}^{-1} \delta_{a b}, \\
& \mathcal{C}_{a}^{b} \stackrel{*}{=}-\frac{\omega v c^{2}}{2} \delta_{a}^{b}, \quad \mathcal{D}_{b}^{a} \stackrel{*}{=} \frac{\omega v c^{2}}{2} \delta_{b}^{a} .
\end{aligned}
$$

Here we have also used $\eta_{00} \stackrel{*}{=} c^{2}, \eta_{a b} \stackrel{*}{=}-\delta_{a b}, \eta^{00} \stackrel{*}{=} c^{-2}$ and $\eta^{a b} \stackrel{*}{=}-\delta^{a b}$. 
With this constitutive matrices, the $3+1$ decomposition of the constitutive law takes the form

$$
\mathcal{D}^{a} \stackrel{*}{=} \varepsilon_{0} \delta^{a b} E_{b}+\frac{\omega v c^{2}}{2} B^{a}, \quad \mathcal{H}_{a} \stackrel{*}{=} \frac{\omega v c^{2}}{2} E_{a}+\mu_{0}^{-1} \delta_{a b} B^{b},
$$

or, in the vector notation used by Nieves and Pal $[57,58]$,

$$
\mathbf{D} \stackrel{*}{=} \epsilon_{0} \mathbf{E}+\gamma \mathbf{B}, \quad \mathbf{H} \stackrel{*}{=} \gamma \mathbf{E}+\mu_{0}^{-1} \mathbf{B} .
$$

Therefore, the constitutive relation of Nieves and Pal can be recovered provided $\gamma=\omega v c^{2} / 2$.

Since we have introduced an additional timelike vector, it is natural that the material will violate $T$ invariance, since $v$ defines a preferred 'time direction'.

Another example is found by considering $S_{a} \stackrel{\text { }}{=} n_{a}, S_{0}{ }^{a} \stackrel{*}{=} 0, S_{0}{ }^{0} \stackrel{*}{=} 0$ and $S_{a}^{b} \stackrel{*}{=} 0$. In this case, we obtain

$$
\begin{aligned}
& \mathcal{A}^{b a} \stackrel{*}{=}-\varepsilon_{0} \delta^{a b}+\epsilon^{a b c} n_{c}, \quad \mathcal{B}_{b a} \stackrel{*}{=} \mu_{0}^{-1} \delta_{a b}, \\
& \mathcal{C}_{a}^{b} \stackrel{*}{=} 0, \quad \mathcal{D}_{b}{ }^{a} \stackrel{*}{=} 0 .
\end{aligned}
$$

and therefore

$$
\mathbf{D} \stackrel{*}{=} \epsilon_{0} \mathbf{E}+\mathbf{n} \times \mathbf{E}, \quad \mathbf{H} \stackrel{*}{=} \mu_{0}^{-1} \mathbf{B} .
$$

This kind of constitutive tensor has the same algebraic form as that considered by Nieves and Pal in [57]. The $P$-violating term they considered can be reproduces if one takes $n_{a} \stackrel{*}{=} i \varepsilon_{p} \hat{k}_{a}$ where $\hat{k}_{a}$ are the components of their wave vector, see [57].

\subsubsection{Nonlinear constitutive relations}

\section{Heisenberg-Euler constitutive relation}

Even when the classical vacuum constitutive relation (i.e. the classical spacetime relation) is linear in Maxwell-Lorentz electrodynamics, its quantum version, QED, predicts corrections due to photon interaction with the quantum vacuum. The effects of this interaction (e.g., vacuum polarization) can be phenomenologically described in a classical theory by means of a nonlinear constitutive law. This effective constitutive law was derived by Heisenberg 
and Euler [33] and can be written, to second order in the fine structure constant $\alpha_{\mathrm{f}}=\frac{e^{2}}{4 \pi \varepsilon_{0} \hbar c}$, as $[33,35,74]$

$$
\begin{aligned}
H=\sqrt{\frac{\varepsilon_{0}}{\mu_{0}}}\{[1 & \left.+\frac{32 \pi}{45} \frac{\alpha_{\mathrm{f}}^{2} \hbar^{3} \varepsilon_{0}}{m^{4} c^{3}} *\left(F \wedge{ }^{*} F\right)\right]{ }^{*} F \\
& \left.+\frac{56 \pi}{45} \frac{\alpha_{\mathrm{f}}^{2} \hbar^{3} \varepsilon_{0}}{m^{4} c^{3}} *(F \wedge F) F\right\}
\end{aligned}
$$

where $m$ is the mass of the electron.

The nonlinear terms describe then the vacuum polarizability due to the interaction of a classical electromagnetic field with the quantum fluctuations of the electron-positron field.

\section{Born-Infeld electrodynamics}

Born-Infeld electrodynamics [5] is a classical nonlinear generalization of Maxwell electrodynamics. The spacetime relation for this model can be written as

$$
H=\sqrt{\frac{\varepsilon_{0}}{\mu_{0}}} \frac{{ }^{*} F-{\frac{1}{2 f^{2}}}^{*}(F \wedge F) F}{\sqrt{\left.1+{\frac{1}{f^{2}}}^{*}\left(F \wedge \wedge^{*} F\right)-\frac{1}{4 f^{4}}{ }^{*}(F \wedge F)\right]^{2}}} .
$$

Here $f$ is a dimensionfull parameter, $[f]=h / q$, the so-called maximal field strength. One of the particular features of this model is that, due to the nonlinearity of the spacetime relation, the electric field corresponding to a point particle (an 'electron') does not follows the usual $1 / r^{2}$ law which, in particular, implies that the field is singular at $r=0$. In fact, in the Born-Infeld theory the electric field reaches a maximum value $E_{\max }=c f$. Maxwell-Lorentz electrodynamics is recovered at the limit $f \rightarrow \infty$. One can consider $E_{\max } \approx e / 4 \pi \varepsilon_{0} r_{\mathrm{e}}^{2}$ where $r_{\mathrm{e}}$ denotes the 'classical' electron radius, $r_{\mathrm{e}}=\alpha_{\mathrm{f}} \hbar / m c$. Then the differences between the usual Maxwell-Lorentz electrodynamics and the Born-Infeld model are important only at scales of the order $r_{\mathrm{e}} \approx 2.82 \times 10^{-15} \mathrm{~m}$. However, since the model is nonlinear, no superposition principle is valid and the propagation of waves depends on the field configuration, see 3.4 for more details.

\subsubsection{Effective constitutive tensor}

Consider an arbitrary local relation $H=H(F)$. The corresponding Maxwell equation in vacuum is written, in components, as

$$
\partial_{j}\left(\epsilon^{i j k l} H_{k l}\right)=0 \text {. }
$$


Consider now the properties of a small perturbation $\Delta F$ of the electromagnetic field around some background configuration $\bar{F}$. We write the total electromagnetic field strength $F$ as $F=\bar{F}+\Delta F$. Then the field excitation can be written, to first order in the perturbation $\Delta F$, as

$$
H_{i j}(F)=H_{i j}(\bar{F})+\left.\frac{1}{2} \frac{\partial H_{i j}}{\partial F_{k l}}\right|_{\bar{F}} \Delta F_{k l} .
$$

Inserting (2.99) into (2.98) and assuming that the background field $\bar{F}$ is a solution of $(2.98)$, i.e. $\partial_{j}\left[\epsilon^{i j k l} H_{k l}(\bar{F})\right]=0$, we obtain an equation for the perturbation:

$$
\partial_{j}\left(\left.\frac{1}{2} \epsilon^{i j k l} \frac{\partial H_{k l}}{\partial F_{m n}}\right|_{\bar{F}} \Delta F_{m n}\right)=0 .
$$

We can write this equation in the same form as in the linear case, i.e. as

$$
\partial_{j}\left(\chi_{\mathrm{eff}}^{i j k l} \Delta F_{k l}\right)=0
$$

with the 'effective constitutive tensor'

$$
\chi_{\mathrm{eff}}^{i j k l}:=\left.\frac{1}{2} \epsilon^{i j m n} \frac{\partial H_{m n}}{\partial F_{k l}}\right|_{\bar{F}} .
$$

The tensor $\chi_{\text {eff }}^{i j k l}$ will, in general, depend on the local constitutive law and on the background field $\bar{F}$. This result shows that most of the results obtained for linear constitutive/spacetime relations can also be applied to every local electromagnetic theory provided one considers perturbations on some background solution. This is the case of the propagation of waves in nonlinear media, e.g., where the waves are precisely weak perturbations on a given field configuration. 


\subsection{Symmetries and Energy-momentum}

\subsubsection{Symmetry of a linear medium}

Here I would like to define the concept of a symmetry of the medium. This definition applies to the linear case, when a constitutive tensor $\chi$ is available.

To motivate our definition, consider the particular case in which the field configuration is such that the Lie-derivative of the electromagnetic field strength along some vector field $\xi$ vanishes, i.e.

$$
\mathcal{L}_{\xi} F=0
$$

The geometric interpretation of the condition (2.103) is clear. It means that the electromagnetic field strength $F$ is 'constant' along the direction $\xi$. More precisely, the integral $\int_{\Omega_{2}} F$ on some 2-dimensional surface $\Omega_{2}$ (which is the natural physical quantity associated to the field) is invariant under the displacement of the integration region $\Omega_{2}$ induced by $\xi$. This property follows from the very definition of the Lie derivative, see, for instance, the discussion in [72] and figure 2.4 for $\Psi=F$ and $n=\xi$.

Now, in linear electrodynamics the excitation is determined by the field strength $F$ and the constitutive tensor $\chi$. It is clear that even if (the integral of) the field strength $F$ is constant under the displacements defined by $\xi$, i.e., if (2.103) holds, the excitation will not be constant unless the medium itself, i.e. the constitutive tensor, satisfies some condition. We will call this condition a symmetry condition for the medium. Clearly, the condition we are referring to is the vanishing of the Lie derivative of the constitutive tensor, since in this case (2.103) implies that $H$ is also constant, i.e. $\mathcal{L}_{\xi} H=0$. This lead us to the following definition:

Definition: A linear medium is said to have a symmetry under the displacement induced by a vector field $\xi$ if the Lie derivative along $\xi$ of its constitutive tensor vanishes, i.e. if

$$
\mathcal{L}_{\xi} \chi^{i j k l}=0
$$

for some vector field $\xi$.

For the explicit expression of the Lie derivative of the tensor density $\chi$ one should compare appendix C.2, equation (C.6). Notice that this condition implies the independent vanishing of the Lie derivative of each irreducible piece of the constitutive tensor, see the general decomposition formula (2.47). 
From this definition and the property about the noncommutativity of the Lie derivative, it follows that if $\xi_{1}$ and $\xi_{2}$ are two vector fields describing symmetries of the medium, then the new vector $\left[\xi_{1}, \xi_{2}\right]$ is also a symmetry of the medium, see appendix C.2 for more details.

This immediately raises questions analogous to those one encounters in the study of isometries in GR, namely (a) what is the maximum number $n_{\max }$ of symmetries that a linear medium allows? And then, assuming that $n_{\max }$ is finite, (b) what is the form of a 'maximally symmetric' constitutive tensor?, i.e., of a constitutive tensor allowing the maximum number of symmetries?

I do not have at this moment the answers to these questions. However, it can be speculated that maybe the maximally symmetric constitutive tensor can correspond to $\chi=\chi_{\eta}$, i.e., to the (conformal) Minkowski vacuum, since we know that it is a highly symmetric case, see also the particular case below. This result would be interesting since it would provide a further way to 'derive' the vacuum spacetime relation by postulating that it has to be maximally symmetric. These questions will be investigated in the future.

\section{Riemannian case}

Here I show that in the special case for which $\chi=\chi_{\{g\}}$, i.e. for the 'vacuum' on a riemannian space ${ }^{8}$, the equation (2.104) defining the symmetries of the medium is equivalent to the conformal Killing equation for the metric $g$, i.e. to

$$
\mathcal{L}_{\xi}\left(|g|^{1 / 4} g^{i j}\right)=0
$$

or, explicitly

$$
g^{m j} \partial_{m} \xi^{i}+g^{i m} \partial_{m} \xi^{j}+\xi^{m} \partial_{m} g^{i j}=\frac{1}{4} g^{i j}\left[2 \partial_{m} \xi^{m}+\xi^{m} g_{k l} \partial_{m} g^{k l}\right] .
$$

One proves this as follows. From (4.3) one finds

$$
\begin{aligned}
\mathcal{L}_{\xi} \chi_{\{g\}}^{i j k l}= & 2\left(|g|^{1 / 4} g^{k[i}\right) \mathcal{L}_{\xi}\left(|g|^{1 / 4} g^{j] l}\right) \\
& +2\left(|g|^{1 / 4} g^{l[j}\right) \mathcal{L}_{\xi}\left(|g|^{1 / 4} g^{i] k}\right) .
\end{aligned}
$$

Contracting (2.107) with $\left(|g|^{-1 / 4} g_{j l}\right)$, one obtains

$$
\left(|g|^{-1 / 4} g_{j l}\right) \mathcal{L}_{\xi} \chi_{\{g\}}^{i j k l}=g^{i k} g_{j l} \mathcal{L}_{\xi}\left(|g|^{1 / 4} g^{j l}\right)+2 \mathcal{L}_{\xi}\left(|g|^{1 / 4} g^{i k}\right)
$$

\footnotetext{
${ }^{8}$ or a material medium in Minkowski space with an 'effective metric' $g$.
} 
so that the condition (2.104) implies

$$
g^{i k} g_{j l} \mathcal{L}_{\xi}\left(|g|^{1 / 4} g^{j l}\right)+2 \mathcal{L}_{\xi}\left(|g|^{1 / 4} g^{i k}\right)=0 .
$$

Contracting this equation with $g_{i k}$ one obtains $g_{j l} \mathcal{L}_{\xi}\left(|g|^{1 / 4} g^{j l}\right)=0$ which, when substituted back into (2.109), results in the conformal Killing equation for the metric $g$, namely (2.105). This means that the conformal Killing equation is a necessary condition for $\xi$ to be a symmetry of the medium. On the other hand, from (2.107) one sees that if $\xi$ is a conformal Killing vector then (2.105) is automatically satisfied. Therefore, (2.105) is also a sufficient condition.

In other words, in the riemannian case, all symmetries of the medium are conformal symmetries of the metric.

\subsubsection{Conservation of energy-momentum}

In physics conservation laws play a central role. Among other properties, they allow the definition of conserved quantities in terms of which the description of the system and its evolution becomes simpler. In particular, energy and momentum are quantities associated to any field. In GR and similar theories they are the source of the gravitational field.

In field theory, energy-momentum distribution and flow are described by a covector valued 3 -form, which in the electromagnetic case we will denote as $\Sigma_{\alpha}$. Usually, one also needs an additional vector field $\xi$ to construct a frame-independent 3-form $Q:=\Sigma_{\alpha} \xi^{\alpha}$ following the general scheme 'conserved quantity' $\sim$ 'momentum' $\times$ 'vector field'. The vector field is usually related to some symmetry of the system. As an example of this general scheme recall the case of GR. There, a conserved quantity along the trajectory of a free test particle (i.e. a particle moving along a geodesic) can be defined provided the spacetime admits a symmetry. This symmetry is described by a Killing vector field $\xi_{\mathrm{K}}$, i.e. an isometry of the spacetime metric, so that $\mathcal{L}_{\xi_{\mathrm{K}}} g_{i j}=0$. The corresponding conserved quantity is then given by the projection (contraction) of the momentum ${ }^{9}$ of the test particle of mass ${ }^{10}$ $m, p_{i}:=m g_{i j} \frac{d x^{j}}{d \tau}$, along the direction of the Killing vector i.e. $q:=p_{i} \xi_{\mathrm{K}}^{i}$.

Back to our original problem, the main property that $Q$ has to fulfill in order to be interpreted as energy-momentum is that it has to be related to

\footnotetext{
${ }^{9}$ Momentum is always a 1 -form, i.e., a covector, see, for instance, figure 2 in [72].

${ }^{10}$ For a photon, use simply $p_{i}=g_{i j} \frac{d x^{j}}{d \tau}$.
} 
the corresponding force law through a derivative of the form $\xi^{\alpha} f_{\alpha} \sim d Q$. If, for instance, $\xi$ happens to be timelike, $\xi^{\alpha} f_{\alpha}$ can be interpreted as the rate of energy transfer to the particles via the Lorentz force. A second condition on $Q$ is that it must be conserved under some conditions, i.e. $d Q=0$. One expects again the condition for energy-momentum conservation to be related to the symmetries of the system, at least when a Lagrangian is available, as we know from the Noether theorem. In linear electrodynamics this latter can happen when the irreducible piece ${ }^{(2)} \chi$ of the constitutive tensor vanishes.

Therefore, to find an adequate energy-momentum $\Sigma_{\alpha}$ for the electromagnetic field, we try to express $\xi^{\alpha} f_{\alpha}$ as a total derivative of some 3 -form $d Q$, with $Q:=\Sigma_{\alpha} \xi^{\alpha}$.

First, we use the expression for the Lorentz force law (2.6), replace the current $J$ from the inhomogeneous Maxwell equation (2.4) and 'partially integrate'. One finds

$$
\begin{aligned}
\xi^{\alpha} f_{\alpha} & \left.=\xi^{\alpha}\left(e_{\alpha}\right\rfloor F\right) \wedge J \\
& =(\xi\rfloor F) \wedge J \\
& =(\xi\rfloor F) \wedge d H \\
& =-d[(\xi\rfloor F) \wedge H]+H \wedge d(\xi\rfloor F) \\
& =-d[(\xi\rfloor F) \wedge H]+H \wedge\left(\mathcal{L}_{\xi} F\right) .
\end{aligned}
$$

In the last step we have used the definition of the Lie derivative of $F$ and the homogenous Maxwell equation, $d F=0$.

The choice of $Q$ that equation (2.110) suggests, namely $Q=-(\xi\rfloor F) \wedge H$, is not what we are looking for since it would lead to conservation of $Q$ only if the field strength is constant in the direction $\xi$, i.e. if $\mathcal{L}_{\xi} F=0$, which is a too restrictive condition.

To improve the situation we use now the fact that (2.110) can be rewritten as

$$
\left.\xi^{\alpha} f_{\alpha}=d[Z-(\xi\rfloor F) \wedge H\right]+H \wedge\left(\mathcal{L}_{\xi} F\right)-d Z,
$$

where $Z$ is an arbitrary 3 -form.

To be sure that one is covering all the available possibilities, one should consider the most general 3 -form $Z$ that can be constructed using the available objects. In our case, they are only $F, H$ and $\xi^{11}$. Under these conditions,

\footnotetext{
${ }^{11}$ In principle, one could also use the potential $A$ as a further object. However, it seems that no 3-form linear in $\xi$, as required by the left hand side of (2.111), can be constructed using this 1-form together with $F$ and $H$.
} 
$Z$ can only be a linear combination of the terms $(\xi\rfloor F) \wedge H$ and $(\xi\rfloor H) \wedge F$. The other 3-form that can be easily constructed, namely $\xi\rfloor(F \wedge H)$, is a particular linear combination of the two former terms. Therefore, we take the ansatz

$$
\left.\left.Z=a_{1}(\xi\rfloor F\right) \wedge H+a_{2}(\xi\rfloor H\right) \wedge F,
$$

with arbitrary constants $a_{1}$ and $a_{2}$. This implies

$$
\begin{aligned}
d Z= & \left.\left.\left.a_{1} d(\xi\rfloor F\right) \wedge H-a_{1}(\xi\rfloor F\right) \wedge d H+a_{2} d(\xi\rfloor H\right) \wedge F \\
& \left.-a_{2}(\xi\rfloor H\right) \wedge d F \\
= & \left.\left.\left.a_{1} d(\xi\rfloor F\right) \wedge H-a_{1}(\xi\rfloor F\right) \wedge J+a_{2} d(\xi\rfloor H\right) \wedge F \\
= & \left.a_{1}\left(\mathcal{L}_{\xi} F\right) \wedge H-a_{1} \xi^{\alpha} f_{\alpha}+a_{2}\left[\mathcal{L}_{\xi} H-(\xi\rfloor d H\right)\right] \wedge F \\
= & \left.a_{1}\left(\mathcal{L}_{\xi} F\right) \wedge H-a_{1} \xi^{\alpha} f_{\alpha}+a_{2}\left(\mathcal{L}_{\xi} H\right) \wedge F-a_{2}(\xi\rfloor J\right) \wedge F \\
= & a_{1}\left(\mathcal{L}_{\xi} F\right) \wedge H+a_{2}\left(\mathcal{L}_{\xi} H\right) \wedge F+\left(a_{2}-a_{1}\right) \xi^{\alpha} f_{\alpha} .
\end{aligned}
$$

Thus, we rewrite (2.110) as

$$
\begin{aligned}
\xi^{\alpha} f_{\alpha}= & \left.\left.d\left[\left(a_{1}-1\right)(\xi\rfloor F\right) \wedge H+a_{2}(\xi\rfloor H\right) \wedge F\right]+\left(1-a_{1}\right) H \wedge\left(\mathcal{L}_{\xi} F\right) \\
& -a_{2}\left(\mathcal{L}_{\xi} H\right) \wedge F-\left(a_{2}-a_{1}\right) \xi^{\alpha} f_{\alpha} .
\end{aligned}
$$

This expression, which is an identity as soon as $F$ and $H$ satisfy the Maxwell equation and $f_{\alpha}$ is given by (2.6), leads us to define

$$
\begin{aligned}
& \left.\left.Q:=\frac{1}{1-a_{1}+a_{2}}\left[\left(a_{1}-1\right)(\xi\rfloor F\right) \wedge H+a_{2}(\xi\rfloor H\right) \wedge F\right], \\
& X:=\frac{1}{1-a_{1}+a_{2}}\left[\left(1-a_{1}\right) H \wedge\left(\mathcal{L}_{\xi} F\right)-a_{2}\left(\mathcal{L}_{\xi} H\right) \wedge F\right],
\end{aligned}
$$

so that

$$
\xi^{\alpha} f_{\alpha}=d Q+X .
$$

The identity (2.117) summarizes all the possibilities to write $\xi^{\alpha} f_{\alpha}$ as a total derivative of the available fields plus some rest. The problem now is to find suitable values for $a_{1}$ and $a_{2}$ so that $Q$ and $X$ satisfy our requirements. In particular, we need $X$ to vanish under some reasonable circumstances.

Up to here our results in this section are valid for any medium, since the constitutive/spacetime relation has not been used. To make up our minds about a reasonable choice of the constants it is useful to study the particular 
case of linear electrodynamics and compute $X$ explicitly in terms of the field strength and the irreducible pieces of the constitutive tensor.

We use $\mathcal{L}_{\xi} F=\frac{1}{2} \mathcal{L}_{\xi} F_{i j} d x^{i} \wedge d x^{j}$ and similarly for $H, \mathcal{L}_{\xi} H=\frac{1}{2} \mathcal{L}_{\xi} H_{i j} d x^{i} \wedge$ $d x^{j}$. Furthermore, we introduce the abbreviation $a:=\frac{1}{4\left(1-a_{1}+a_{2}\right)}$ and compute

$$
\begin{aligned}
X= & a\left[\left(1-a_{1}\right) H_{i j}\left(\mathcal{L}_{\xi} F_{k l}\right)-a_{2}\left(\mathcal{L}_{\xi} H_{i j}\right) F_{k l}\right] d x^{i} \wedge d x^{j} \wedge d x^{k} \wedge d x^{l} \\
= & a\left[\left(1-a_{1}\right) H_{i j}\left(\mathcal{L}_{\xi} F_{k l}\right)-a_{2}\left(\mathcal{L}_{\xi} H_{i j}\right) F_{k l}\right] \epsilon^{i j k l} \hat{\epsilon} \\
= & 2 a\left[\left(1-a_{1}\right) \mathcal{H}^{k l}\left(\mathcal{L}_{\xi} F_{k l}\right)-a_{2}\left(\mathcal{L}_{\xi} \mathcal{H}^{k l}\right) F_{k l}\right] \hat{\epsilon} \\
= & a\left[\left(1-a_{1}\right) \chi^{k l i j} F_{i j}\left(\mathcal{L}_{\xi} F_{k l}\right)-a_{2}\left(\mathcal{L}_{\xi} \chi^{k l i j} F_{i j}\right) F_{k l}\right] \hat{\epsilon} \\
= & a\left[\left(1-a_{1}\right) \chi^{k l i j} F_{i j}\left(\mathcal{L}_{\xi} F_{k l}\right)-a_{2}\left(\mathcal{L}_{\xi} \chi^{k l i j}\right) F_{i j} F_{k l}\right. \\
& \left.\quad-a_{2} \chi^{k l i j}\left(\mathcal{L}_{\xi} F_{i j}\right) F_{k l}\right] \hat{\epsilon} \\
= & a\left[\left[\left(1-a_{1}\right) \chi^{k l i j}-a_{2} \chi^{i j k l}\right] F_{i j}\left(\mathcal{L}_{\xi} F_{k l}\right)-a_{2}\left(\mathcal{L}_{\xi} \chi^{k l i j}\right) F_{i j} F_{k l}\right] \hat{\epsilon} \\
= & a\left[\left(1-a_{1}-a_{2}\right)\left({ }^{(1)} \chi^{k l i j}+{ }^{(3)} \chi^{k l i j}\right) F_{i j}\left(\mathcal{L}_{\xi} F_{k l}\right)\right. \\
& \quad+\left(1-a_{1}+a_{2}\right)^{(2)} \chi^{k l i j} F_{i j}\left(\mathcal{L}_{\xi} F_{k l}\right) \\
& \left.\quad-a_{2} \mathcal{L}_{\xi}\left({ }^{(1)} \chi^{k l i j}+{ }^{(3)} \chi^{k l i j}\right) F_{i j} F_{k l}\right] \hat{\epsilon} .
\end{aligned}
$$

Therefore, we find

$$
\begin{aligned}
X=\frac{1}{4} F_{i j}[ & \frac{\left(1-a_{1}-a_{2}\right)}{\left(1-a_{1}+a_{2}\right)}\left({ }^{(1)} \chi^{k l i j}+{ }^{(3)} \chi^{k l i j}\right)\left(\mathcal{L}_{\xi} F_{k l}\right) \\
& +{ }^{(2)} \chi^{k l i j}\left(\mathcal{L}_{\xi} F_{k l}\right) \\
& \left.-\frac{a_{2}}{\left(1-a_{1}+a_{2}\right)} \mathcal{L}_{\xi}\left({ }^{(1)} \chi^{k l i j}+{ }^{(3)} \chi^{k l i j}\right) F_{k l}\right] \hat{\epsilon}
\end{aligned}
$$

From this result, one recognizes first that no choice of $a_{1}$ and $a_{2}$ makes $X$ to vanish in general. Thus, no conservation law is possible, unless additional conditions are fulfilled. This is, however, what one expects. One also sees that the irreducible pieces enter with different weighting factors in the term $F_{i j}\left(\mathcal{L}_{\xi} F_{k l}\right)$. In particular, notice that the factor $\left(1-a_{1}+a_{2}\right)$ in front of the irreducible piece ${ }^{(2)} \chi$ cancels completely. This means that the term ${ }^{(2)} \chi^{k l i j} F_{i j}\left(\mathcal{L}_{\xi} F_{k l}\right)$ will always be present on the decomposition of $X$, no matter which values of $a_{1}$ and $a_{2}$ one chooses. In other words, the irreducible piece ${ }^{(2)} \chi$ of the constitutive tensor will always prevent $Q$ from being a conserved quantity (in charge-free regions), unless of course a much more restrictive condition, like $\mathcal{L}_{\xi} F=0$, is satisfied by the field configuration. This result 
is consistent with our interpretation of ${ }^{(2)} \chi$ as related to intrinsic dissipative properties of the medium.

The 'best' one can do is to choose

$$
1-a_{1}-a_{2}=0
$$

so that the terms proportional to the symmetric irreducible pieces ${ }^{(1)} \chi$ and ${ }^{(3)} \chi$ in the first term on the right hand side of (2.119) vanish. In this case, after substituting $a_{2}=1-a_{1}$ into (2.115) and (2.116) one finds that $Q$ and $X$ do not depend on $a_{1}$, so that one obtains a unique result, namely

$$
\begin{aligned}
& \left.\left.Q=\frac{1}{2}[(\xi\rfloor H) \wedge F-(\xi\rfloor F\right) \wedge H\right], \\
& X=\frac{1}{2}\left[H \wedge\left(\mathcal{L}_{\xi} F\right)-\left(\mathcal{L}_{\xi} H\right) \wedge F\right] .
\end{aligned}
$$

Furthermore, for linear media we obtain, from (2.119),

$$
X=-\frac{1}{8} F_{i j}\left[2^{(2)} \chi^{i j k l}\left(\mathcal{L}_{\xi} F_{k l}\right)+\mathcal{L}_{\xi}\left({ }^{(1)} \chi^{i j k l}+{ }^{(3)} \chi^{i j k l}\right) F_{k l}\right] \hat{\epsilon} .
$$

That the choice (2.121) and (2.122) is reasonable can be seen as follows. Consider the case in which ${ }^{(2)} \chi=0$ so that a Lagrangian is available for the description of the system. In a charge-free region, (2.123) shows that $Q$ is conserved provided $\xi$ is a symmetry of the medium, i.e. $\mathcal{L}_{\xi} \chi^{k l i j}=0$. Furthermore, our choice agrees with the results valid in a Riemannian space.

Notice also that $Q$ does not depend on the axion-like piece ${ }^{(3)} \chi$ entering the linear constitutive law. This can directly be seen using (2.121) and (2.60), since

$$
\begin{aligned}
\left.\left.(\xi\rfloor^{(3)} H\right) \wedge F-(\xi\rfloor F\right) \wedge{ }^{(3)} H & =(\xi\rfloor(\alpha F)) \wedge F-(\xi\rfloor F) \wedge(\alpha F) \\
& =\alpha(\xi\rfloor F) \wedge F-\alpha(\xi\rfloor F) \wedge F \\
& =0
\end{aligned}
$$

However, the axion-like piece does appear in (2.123) and therefore can contribute to the non-conservation of $Q$ if $\mathcal{L}_{\xi} \alpha \neq 0$. 


\subsubsection{Axiom 4: Energy-momentum tensor}

The results in section 2.7.2 show how to construct a 3 -form which for a linear medium leads to conservation laws, under reasonable conditions. We take these results and generalize them to our fourth axiom, by means of which the kinematic energy-momentum 3 -form ${ }^{\mathrm{k}} \Sigma_{\alpha}$ of any medium is defined by

$$
\left.\left.{ }^{\mathrm{k}} \Sigma_{\alpha}:=\frac{1}{2}\left[F \wedge\left(e_{\alpha}\right\rfloor H\right)-H \wedge\left(e_{\alpha}\right\rfloor F\right)\right] .
$$

Then the 3-form $Q$ of section 2.7.2 is given by $Q={ }^{\mathrm{k}} \Sigma_{\alpha} \xi^{\alpha}$.

The adjective 'kinematic' is used here to emphasize that our definition of ${ }^{\mathrm{k}} \Sigma_{\alpha}$ was not based on dynamical properties of the system, but rather on kinematic arguments.

One can also now define the components ${ }^{\mathrm{k}} \mathcal{T}_{\alpha}{ }^{\beta}$ of the kinetic energymomentum tensor by

$$
\vartheta^{\beta} \wedge{ }^{\mathrm{k}} \Sigma_{\alpha}={ }^{\mathrm{k}} \mathcal{T}_{\alpha}{ }^{\beta} \hat{\epsilon},
$$

where $\hat{\epsilon}:=\vartheta^{\hat{0}} \wedge \vartheta^{\hat{1}} \wedge \vartheta^{\hat{2}} \wedge \vartheta^{\hat{3}}$. In particular, in a coordinate basis, with $\mathcal{H}^{i j}:=\epsilon^{i j k l} H_{k l} / 2$,

$$
{ }^{\mathrm{k}} \mathcal{T}_{i}{ }^{j}=\frac{1}{4} \delta_{i}^{j} F_{k l} \mathcal{H}^{k l}-F_{i k} \mathcal{H}^{j k} .
$$

For a general medium, we summarize our results as

$$
\xi^{\alpha} f_{\alpha}=d Q+X,
$$

with

$$
\begin{aligned}
Q & :={ }^{\mathrm{k}} \Sigma_{\alpha} \xi^{\alpha}, \\
X & =\frac{1}{2}\left[H \wedge\left(\mathcal{L}_{\xi} F\right)-\left(\mathcal{L}_{\xi} H\right) \wedge F\right] .
\end{aligned}
$$

For a linear medium, we notice first that the axion-like piece ${ }^{(3)} \chi$ does not contributes to the kinematic energy-momentum tensor, i.e.,

$$
{ }^{\mathrm{k}} \Sigma_{\alpha}(\chi)={ }^{\mathrm{k}} \Sigma_{\alpha}\left({ }^{(1)} \chi+{ }^{(2)} \chi+{ }^{(3)} \chi\right)={ }^{\mathrm{k}} \Sigma_{\alpha}\left({ }^{(1)} \chi+{ }^{(2)} \chi\right),
$$

as can be seen from the linear dependence of ${ }^{\mathrm{k}} \Sigma$ on the constitutive tensor, see (2.125), and from (2.124). 
Additionally, we have shown, see (2.119) and (2.128), that the corresponding global conservation law takes the form

$$
\begin{aligned}
d Q= & f_{\alpha} \xi^{\alpha} \\
& +\frac{1}{8} F_{i j}\left[\mathcal{L}_{\xi}\left({ }^{(1)} \chi^{i j k l}+{ }^{(3)} \chi^{i j k l}\right) F_{k l}+2^{(2)} \chi^{i j k l}\left(\mathcal{L}_{\xi} F_{k l}\right)\right] \hat{\epsilon},
\end{aligned}
$$

for any vector field $\xi$. In this equation the contributions of the three irreducible pieces ${ }^{(1)} \chi,{ }^{(2)} \chi$, and ${ }^{(3)} \chi$ are isolated from each other.

From these results, a consistent interpretation of (2.132) can be given as a 'balance equation' for the energy-momentum content of the electromagnetic field.

Recall first that even if $\xi$ is a symmetry of the medium, in which case the second and third terms on the right hand side of (2.132) vanish, there is still a contribution proportional to the irreducible piece ${ }^{(2)} \chi$ of the constitutive tensor. This is interpreted as an intrinsic dissipative property of the medium generated by the irreducible piece ${ }^{(2)} \chi$.

In the case that $\xi$ is a timelike field (for instance for a material medium in flat spacetime) the result above generalizes an equation given by Landau $\sim \mathrm{E} \cdot \frac{\partial \mathrm{D}}{\partial t}[45]$ and can be interpreted as an 'energy balance' equation for the change of the total electromagnetic energy. The integration of (2.132) over a 4-dimensional region of the form $\Omega_{4}=\Sigma \times\left[\sigma_{0}, \sigma\right]$ will produce ${ }^{12}$ at the left hand side the 'change of the total energy of the electromagnetic field' between the 'times' $\sigma_{0}$ and $\sigma$, i.e., $\int_{\Sigma_{\sigma}} Q-\int_{\Sigma_{\sigma_{0}}} Q$. The first term on the right hand side, $f_{\alpha} \xi^{\alpha}$, will lead to the energy transfered from the electromagnetic field to the test particles via the Lorentz force, i.e., $\int_{\Omega_{4}} f_{\alpha} \xi^{\alpha}$. The term proportional to ${ }^{(2)} \chi$ can be then interpreted as the rate at which the energy of the electromagnetic field is dissipated, $\frac{1}{4} \int_{\Omega_{4}} F_{i j}{ }^{(2)} \chi^{i j k l}\left(\mathcal{L}_{\xi} F_{k l}\right) \hat{\epsilon}$. The other terms will be proportional to the time derivative of the constitutive tensor, $\frac{1}{8} \int_{\Omega_{4}} F_{i j} \mathcal{L}_{\xi}\left({ }^{(1)} \chi^{i j k l}+{ }^{(3)} \chi^{i j k l}\right) F_{k l} \hat{\epsilon}$. When the medium is time independent this term vanishes. If the time derivative does not vanishes, it means that the properties of the medium are changing in time. Therefore, one could try to interpret this term as the energy per unit time needed to change the material properties ${ }^{13}$.

\footnotetext{
${ }^{12} \mathrm{We}$ assume that the fields vanish at $\partial \Sigma_{\sigma}$ ('spatial infinity').

${ }^{13}$ Maybe with a change of some suitably defined 'internal energy' of the medium.
} 


\section{Chapter 3}

\section{Wave propagation}

We turn our attention to the wave propagation properties in our general framework of linear pre-metric electrodynamics.

Consider a region in spacetime without charges, i.e., $J=0$. Maxwell's equations take then the form

$$
d F=0 \quad d H=0,
$$

completed by the linear constitutive/spacetime relations (2.45). These equations will allow solutions propagating in spacetime, the behavior of which is determined by the properties of the medium/spacetime.

Since the constitutive tensor can have a very complicated spacetime dependence, many specific features of the propagation of waves over finite distances cannot be studied in general terms here. However, an important local property of the propagation of waves, namely the dispersion relation that the covectors tangent to a wave fronts must satisfy, can be derived in general. This is done by deriving the so-called Fresnel equation for the wave covectors.

\subsection{Propagation of singularities}

In the theory of partial differential equations, the propagation of waves is described by Hadamard discontinuities of solutions across a characteristic (wave front) hypersurface $S$ [21]. One can locally define $S$ by the equation $\Phi\left(x^{i}\right)=$ const, for some function $\Phi(x)$. The Hadamard discontinuity of any function $\mathcal{F}(x)$ across the hypersurface $S$ is defined as the difference $[\mathcal{F}]_{S}(x):=\mathcal{F}\left(x_{+}\right)-\mathcal{F}\left(x_{-}\right)$, where $x_{ \pm}:=\lim _{\varepsilon \rightarrow 0}(x \pm \varepsilon)$ are points on the 


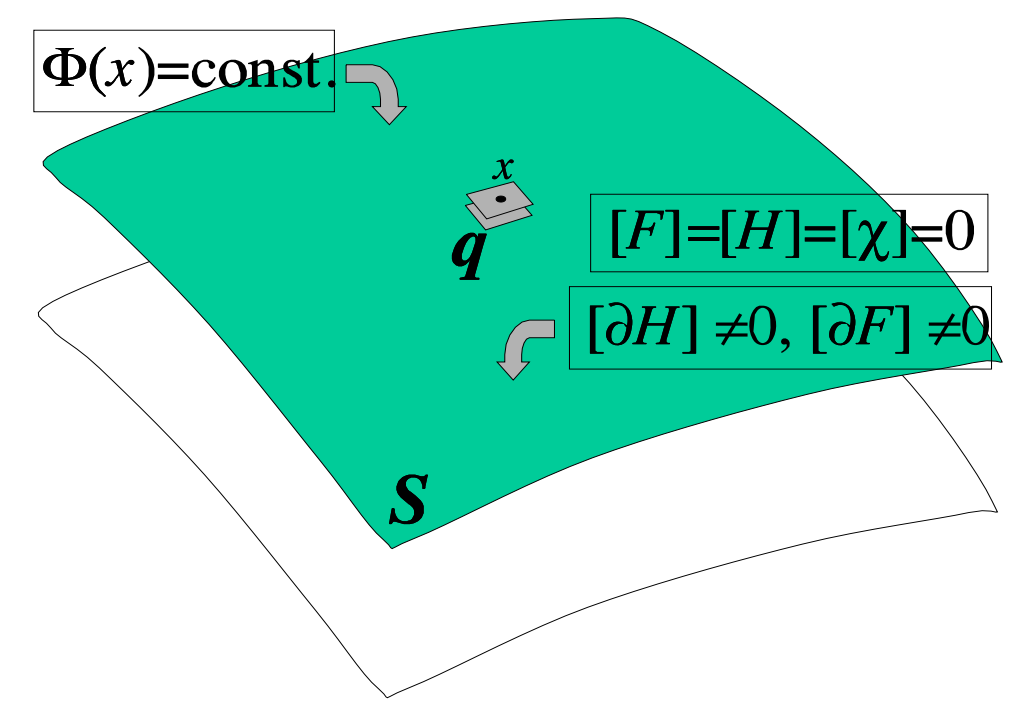

Figure 3.1: Characteristic surface for propagation of electromagnetic disturbances. The 1-form $q:=d \Phi$ corresponds geometrically to two small parallel planes tangent to $S$ at each point $x$.

opposite sides of $S \ni x$. An ordinary electromagnetic wave is a solution of the vacuum Maxwell equations (3.1) for which the derivatives of $H$ and $F$ have discontinuities across the wave front hypersurface $S$.

Thus, in terms of field components, we have on the characteristic hypersurface $S$,

$$
\begin{aligned}
& {\left[F_{i j}\right]_{S}=0, \quad\left[\partial_{i} F_{j k}\right]_{S}=q_{i} f_{j k},} \\
& {\left[H_{i j}\right]_{S}=0, \quad\left[\partial_{i} H_{j k}\right]_{S}=q_{i} h_{j k}}
\end{aligned}
$$

where $f_{i j}$ and $h_{i j}$ are the components of the 2-forms $f$ and $h$ describing the corresponding jumps of the derivatives of field strength and excitation across $S$, respectively, and the covector normal to the wave front is given by

$$
q:=d \Phi=q_{i} d x^{i}
$$

Notice that (3.2) and (3.3), taken together, are covariant conditions. In particular, alltough $(3.2 \mathrm{~b})$ and $(3.3 \mathrm{~b})$ may not look covariant at first sight, they are, provided $(3.2 \mathrm{a})$ and $(3.3 \mathrm{a})$ are also satisfied. This can be seen by considering the transformation law of $\partial_{i} F_{j k}$ under coordinate transformations. 
Consider the coordinate transformation $x^{i} \rightarrow x^{i^{\prime}}$, for example. Then we have

$$
\partial_{i^{\prime}} F_{j^{\prime} k^{\prime}}=\frac{\partial}{\partial x^{i^{i}}}\left(\frac{\partial x^{j}}{\partial x^{j^{\prime}}} \frac{\partial x^{k}}{\partial x^{k^{\prime}}}\right) F_{j k}+\frac{\partial x^{i}}{\partial x^{i^{\prime}}} \frac{\partial x^{j}}{\partial x^{j^{\prime}}} \frac{\partial x^{k}}{\partial x^{k^{\prime}}} \partial_{i} F_{j k} .
$$

Assuming that the coordinate transformation is smooth across $S$, i.e. $\left[\frac{\partial x^{i}}{\partial x^{i}}\right]_{S}=$ $0,\left[\frac{\partial^{2} x^{i}}{\partial x^{i} \partial x^{j}}\right]_{S}=0$, we find that the jump of $\partial_{i} F_{j k}$ across $S$ is given by

$$
\left[\partial_{i^{\prime}} F_{j^{\prime} k^{\prime}}\right]_{S}=\frac{\partial}{\partial x^{i^{\prime}}}\left(\frac{\partial x^{j}}{\partial x^{j^{\prime}}} \frac{\partial x^{k}}{\partial x^{k^{\prime}}}\right)\left[F_{j k}\right]_{S}+\frac{\partial x^{i}}{\partial x^{i^{i}}} \frac{\partial x^{j}}{\partial x^{j^{\prime}}} \frac{\partial x^{k}}{\partial x^{k^{\prime}}}\left[\partial_{i} F_{j k}\right]_{S},
$$

which reduces to the transformation law of a tensor field, provided (3.2a) is satisfied, i.e.

$$
\left[\partial_{i^{\prime}} F_{j^{\prime} k^{\prime}}\right]_{S}=\frac{\partial x^{i}}{\partial x^{i^{\prime}}} \frac{\partial x^{j}}{\partial x^{j^{\prime}}} \frac{\partial x^{k}}{\partial x^{k^{\prime}}}\left[\partial_{i} F_{j k}\right]_{S} .
$$

The compatibility conditions on the discontinuities of the solutions of Maxwell's equations are then

$$
q \wedge h=0, \quad q \wedge f=0 .
$$

This ensures that the Maxwell equations (3.1) are satisfied. The Hadamard method used here is equivalent to the usual geometric optics limit made by expanding a solution for the electromagnetic potential of the form $A=a e^{i \Phi}$. The covector $q:=d \Phi$ corresponds thus to the wave covector.

Assuming now that the constitutive tensor is regular on $S$ (in a geometric optics limit this means that we assume the scale of variations of the constitutive tensor to be much bigger that the scale of variation of the wave field) we obtain from (2.45) the corresponding relation between $h$ and $f$, namely

$$
h={ }^{\#} f+\alpha f .
$$

Substituting (3.9) into (3.8a) and using (3.8b), we see that the equations (3.8) reduce to

$$
q \wedge{ }^{\#} f=0, \quad q \wedge f=0,
$$

showing that the pseudo-scalar field $\alpha$ drops out completely. Thus it will not contribute to the Fresnel equation. This is no big surprise since we saw that only inhomogeneities of $\alpha$ contribute to Maxwell's equations, and the 
assumption of regularity of the constitutive tensor, i.e.., $[\alpha]_{S}=0$, means that $\alpha$ does not have discontinuities across $S$.

Now, the general solution of $(3.10 \mathrm{~b})$ is of the form

$$
f=q \wedge a
$$

where the 1-form $a$ is defined up to 'gauge transformations'

$$
a \rightarrow a+\varphi q
$$

for any scalar function $\varphi$. This is an algebraic consequence of the gauge freedom in the definition of the electromagnetic potential. Then the compatibility condition (3.10) reduces to

$$
q \wedge \wedge^{\#}(q \wedge a)=0
$$

Since (3.13) is an equation for a 3 -form, it implies four equations that $f$ and $h$ must satisfy in order to allow non-vanishing wave covectors $q$.

Furthermore, in order to allow solutions, the fields $f$ and $h$ must satisfy some 'integrability' conditions. Since the form of the general solutions of (3.8) is $f=q \wedge a$ and $h=q \wedge b$, we find

$$
f \wedge f=0, \quad h \wedge h=0, \quad f \wedge h=0 .
$$

or, in components,

$$
\epsilon^{i j k l} f_{i j} f_{k l}=0, \quad \epsilon^{i j k l} h_{i j} h_{k l}=0, \quad \epsilon^{i j k l} f_{i j} h_{k l}=0 .
$$

\subsection{Fresnel equation}

As has been noticed before, see [62], not all the equations in (3.13) are independent. This fact makes the derivation of the Fresnel equation more involved.

In order to isolate the trivial parts of (3.13), we use a covector basis $\vartheta^{\alpha}$ to write the covector $a$ in terms of its frame components $a_{\alpha}$ as $a=a_{\alpha} \vartheta^{\alpha}$ and choose the covector $\vartheta^{\hat{0}}$ as the covector $q$, i.e. $\vartheta^{\hat{0}}=q$. This can, of course, always be done at each point of spacetime, since we assume $q \neq$ 0 . With this choice, a gauge transformation (3.12) can then be completely accounted for by a transformation $a_{\hat{0}} \rightarrow a_{\hat{0}}+\varphi$. This means that the other frame components $a_{A}, A, B, \ldots=\hat{1}, \hat{2}, \hat{3}$, are gauge invariant. Since the term 
coming from the basis covector $\vartheta^{\hat{0}}$ identically vanishes, i.e. $q \wedge{ }^{\#}\left(q \wedge \vartheta^{\hat{0}}\right) \equiv 0$, (3.13) can be written as

$$
q \wedge^{\#}\left(q \wedge \vartheta^{B}\right) a_{B}=0,
$$

Furthermore, only 3 of the 4 components in the 3-form (3.16) are non-trivial, since multiplying (3.16) by $\vartheta^{\hat{0}}$ one finds

$$
\vartheta^{\hat{0}} \wedge q \wedge{ }^{\#}\left(q \wedge \vartheta^{B}\right) a_{B} \equiv 0 .
$$

The remaining 3 components are

$$
\vartheta^{A} \wedge q \wedge{ }^{\#}\left(q \wedge \vartheta^{B}\right) a_{B}=0 .
$$

This equation can be written as

$$
\hat{\epsilon} W^{A B} a_{B}=0,
$$

with a volume element $\hat{\epsilon}$ that we choose to be $\hat{\epsilon}:=\vartheta^{\hat{0}} \wedge \vartheta^{\hat{1}} \wedge \vartheta^{\hat{2}} \wedge \vartheta^{\hat{3}}$ (it doesn't matter very much which 4 -form one uses, since the equation is homogeneous) and a $3 \times 3$ matrix $W^{A B}$. Using (2.62) and (3.18) we compute

$$
\begin{aligned}
\vartheta^{A} \wedge q \wedge{ }^{\#}\left(q \wedge \vartheta^{B}\right) & =\frac{1}{4} \vartheta^{A} \wedge \vartheta^{\hat{0}} \wedge\left(\hat{\epsilon}_{\alpha \beta \gamma \delta} \chi^{\gamma \delta \epsilon \theta} \delta_{\epsilon}^{\hat{0}} \delta_{\theta}^{B} \vartheta^{\alpha} \wedge \vartheta^{\beta}\right) \\
& =\frac{1}{4} \epsilon^{A \hat{0} \alpha \beta} \hat{\epsilon}_{\alpha \beta \gamma \delta} \chi^{\gamma \delta \hat{o} B} \vartheta^{\hat{0}} \wedge \vartheta^{\hat{1}} \wedge \vartheta^{\hat{2}} \wedge \vartheta^{\hat{3}} \\
& =\delta_{[\gamma}^{A} \delta_{\delta]}^{\hat{0}} \chi^{\gamma \delta \hat{0} B} \hat{\epsilon} \\
& =\chi^{A \hat{0} \hat{0} B} \hat{\epsilon} \\
& =-\chi^{\hat{0} A \hat{0} B} \hat{\epsilon} .
\end{aligned}
$$

Thus, since the negative sign is unimportant, we can define,

$$
W^{A B}:=\chi^{\hat{0} A \hat{0} B} .
$$

The corresponding Fresnel equation is then obtained from the vanishing of the determinant of $W$, as the necessary and sufficient condition for existence of non-vanishing solutions $a_{B}$. Now, since $W$ is a $3 \times 3$ matrix, its determinant is given by

$$
\begin{aligned}
\mathcal{W} & :=\operatorname{det}(W) \\
& =\frac{1}{3 !} \hat{\epsilon}_{A B C} \hat{\epsilon}_{D E F} W^{A D} W^{B E} W^{C F} \\
& =\frac{1}{3 !} \hat{\epsilon}_{A B C} \hat{\epsilon}_{D E F} \chi^{\hat{0} A \hat{0} D} \chi^{\hat{0} B \hat{0} E} \chi^{\hat{0} C \hat{0} F}
\end{aligned}
$$


We apply now the following procedure to rewrite $\mathcal{W}$ as a fully 4 -dimensional covariant expression. We 'complete' the 3-dimensional Levi-Civita symbols, e.g. $\hat{\epsilon}_{A B C}$ to obtain the 4-dimensional one, by first using $\hat{\epsilon}_{A B C} \equiv \hat{\epsilon}_{\hat{0} A B C}$ and then using one of the $\hat{0}$-components of the constitutive tensors as fourth summation index. This leads us to consider the following identity:

$$
\hat{\epsilon}_{\hat{0} A B C} \chi^{\hat{0} A \hat{0} D} \chi^{\hat{0} B \hat{0} E} \chi^{\hat{0} C \hat{0} F} \equiv \frac{1}{2} \hat{\epsilon}_{\alpha \beta \gamma \delta} \chi^{\alpha \beta \hat{0} D} \chi^{\hat{0} \gamma \hat{0} E} \chi^{\hat{0} \delta \hat{0} F}
$$

This identity holds because on the right hand side of (3.23), due to the properties of the Levi-Civita symbol, one of the indices $\alpha, \beta, \gamma$ or $\delta$ must be zero, but on the other hand only $\alpha$ and $\beta$ will contribute, due to the (anti)symmetry properties of the constitutive tensor, see (2.46), which would otherwise make one of the two last $\chi$-factors vanish. Finally, the two remaining contributions are equal, canceling the factor $1 / 2$ and proving the identity. This allows us to rewrite $(3.22)$ as

$$
\mathcal{W}=\frac{1}{3 !} \frac{1}{2} \hat{\epsilon}_{\alpha \beta \gamma \delta} \hat{\epsilon}_{D E F} \chi^{\alpha \beta \hat{0} D} \chi^{\hat{0} \gamma \hat{0} E} \chi^{\hat{0} \delta \hat{0} F}
$$

We repeat the same procedure to complete the remaining 3-dimensional LeviCivita symbol. Now, we can use the following identity:

$$
\hat{\epsilon}_{\hat{0} D E F} \chi^{\alpha \beta \hat{0} D} \chi^{\hat{0} \gamma \hat{0} E} \chi^{\hat{0} \delta \hat{0} F} \equiv \frac{1}{2} \hat{\epsilon}_{\lambda \rho \sigma \tau} \chi^{\alpha \beta \hat{0} \rho} \chi^{\hat{0} \gamma \hat{0} \sigma} \chi^{\hat{0} \delta \lambda \tau} \text {. }
$$

Using (3.25) in (3.24), we finally obtain

$$
\mathcal{W}=\frac{1}{4 !} \hat{\epsilon}_{\alpha \beta \gamma \delta} \hat{\epsilon}_{\lambda \rho \sigma \tau} \chi^{\alpha \beta \hat{0} \rho} \chi^{\hat{0} \gamma \hat{0} \sigma} \chi^{\hat{0} \delta \lambda \tau}
$$

Since $\vartheta^{\hat{0}}=q=q_{i} d x^{i}$, the above result can be written in coordinate components as

$$
\mathcal{W}=\frac{\theta^{2}}{4 !} \hat{\epsilon}_{m n p q} \hat{\epsilon}_{r s t u} \chi^{m n r i} \chi^{j p s k} \chi^{l q t u} q_{i} q_{j} q_{k} q_{l}
$$

with $\theta:=\operatorname{det}\left(e_{i}^{\alpha}\right)$. We define the fourth order tensor density of weight +1 , the 'Fresnel tensor' $\mathcal{G}^{i j k l}$, as

$$
\mathcal{G}^{i j k l}:=\frac{1}{4 !} \hat{\epsilon}_{m n p q} \hat{\epsilon}_{r s t u} \chi^{m n r(i} \chi^{j|p s| k} \chi^{l) q t u}, \quad \mathcal{G}^{i j k l}=\mathcal{G}^{(i j k l)}
$$


which has 35 independent components. Then the Fresnel equation is written finally as

$$
\mathcal{G}^{i j k l} q_{i} q_{j} q_{k} q_{l}=0 .
$$

At each point, the Fresnel equation (3.29) defines in the space of wave covectors the wave (co-)vector surface, see for instance [45]. I would like to emphasize the generality of the above result. The Fresnel equation (3.29) is valid for any linear medium. This means for media that in general are inhomogeneous, anisotropic and dissipative. As we have already discussed, this result can even be applied for the propagation of perturbation of any local medium, by using an efective constitutive tensor.

The general Fresnel equation (3.29) is always a quartic equation in $q_{i}$ despite the fact that it was derived from a determinant of a $3 \times 3$ matrix quadratic in the wave covectors. This is because the remaining quadratic term factorizes in the trivial factor $\theta^{2}$. This corrects Denisov \& Denisov [14] who claim that a particular case of the general linear constitutive law may yield a sixth order Fresnel equation; in [44], even a Fresnel equation of eighth order is claimed to hold.

\subsubsection{Coordinate $3+1$ decomposition of the Fresnel equation}

We rewrite now our general result $(3.27)$ by performing a $3+1$ coordinate decomposition, and obtain

$$
\begin{gathered}
\mathcal{W}=\theta^{2}\left(q_{0}^{4} M+q_{0}^{3} q_{a} M^{a}+q_{0}^{2} q_{a} q_{b} M^{a b}+q_{0} q_{a} q_{b} q_{c} M^{a b c}\right. \\
\left.+q_{a} q_{b} q_{c} q_{d} M^{a b c d}\right)
\end{gathered}
$$

with

$$
\begin{aligned}
& M:=\mathcal{G}^{0000}, \quad M^{a}:=4 \mathcal{G}^{000 a}, \quad M^{a b}:=6 \mathcal{G}^{00 a b}, \\
& M^{a b c}:=4 \mathcal{G}^{0 a b c}, \quad M^{a b c d}:=\mathcal{G}^{a b c d}
\end{aligned}
$$

or explicitly in terms of the $3 \times 3$ matrices defined in (2.64) and (2.65):

$$
\begin{aligned}
M & =\operatorname{det} \mathcal{A} \\
M^{a} & =-\hat{\epsilon}_{b c d}\left(\mathcal{A}^{b a} \mathcal{A}^{c e} \mathcal{C}_{e}^{d}+\mathcal{A}^{a b} \mathcal{A}^{e c} \mathcal{D}_{e}{ }^{d}\right)
\end{aligned}
$$




$$
\begin{aligned}
& M^{a b}=\frac{1}{2} \mathcal{A}^{(a b)}\left[\left(\mathcal{C}^{d}{ }_{d}\right)^{2}+\left(\mathcal{D}_{c}{ }^{c}\right)^{2}-\left(\mathcal{C}^{c}{ }_{d}+\mathcal{D}_{d}{ }^{c}\right)\left(\mathcal{C}^{d}{ }_{c}+\mathcal{D}_{c}{ }^{d}\right)\right] \\
& +\left(\mathcal{C}^{d}{ }_{c}+\mathcal{D}_{c}{ }^{d}\right)\left(\mathcal{A}^{c(a} \mathcal{C}^{b)}{ }_{d}+\mathcal{D}_{d}{ }^{(a} \mathcal{A}^{b) c}\right)-\mathcal{C}^{d}{ }_{d} \mathcal{A}^{c(a} \mathcal{C}^{b)}{ }_{c} \\
& -\mathcal{D}_{c}{ }^{(a} \mathcal{A}^{b) c} \mathcal{D}_{d}{ }^{d}-\mathcal{A}^{d c} \mathcal{C}^{(a}{ }_{c} \mathcal{D}_{d}{ }^{b)} \\
& +\left(\mathcal{A}^{(a b)} \mathcal{A}^{d c}-\mathcal{A}^{d(a} \mathcal{A}^{b) c}\right) \mathcal{B}_{d c}, \\
& M^{a b c}=\epsilon^{d e(c \mid}\left[\mathcal{B}_{d f}\left(\mathcal{A}^{a b)} \mathcal{D}_{e}^{f}-\mathcal{D}_{e}{ }^{a} \mathcal{A}^{b) f}\right)+\mathcal{B}_{f d}\left(\mathcal{A}^{a b)} \mathcal{C}^{f}{ }_{e}\right.\right. \\
& \left.\left.-\mathcal{A}^{f(a} \mathcal{C}_{e}^{b)}\right)+\mathcal{C}_{f}^{a} \mathcal{D}_{e}{ }^{b)} \mathcal{D}_{d}{ }^{f}+\mathcal{D}_{f}{ }^{a} \mathcal{C}_{e}^{b)} \mathcal{C}_{d}^{f}\right] \\
& M^{a b c d}=\epsilon^{e f(c} \epsilon^{|g h| d} \mathcal{B}_{h f}\left[\frac{1}{2} \mathcal{A}^{a b)} \mathcal{B}_{g e}-\mathcal{C}^{a}{ }_{e} \mathcal{D}_{g}^{b)}\right] \text {. }
\end{aligned}
$$

Each 3-dimensional tensor $M$ is totally symmetric, i.e. $M^{a b}=M^{(a b)}, M^{a b c}=$ $M^{(a b c)}$, etc. This results have been verified many times using the Maple computer algebra features, together with the tensor package GrTensor ${ }^{1}$.

\subsubsection{Properties of the Fresnel tensor density}

First, as we already mentioned, one notices that the Fresnel equation is independent of the axion-like piece ${ }^{(3)} \chi$ of the constitutive tensor:

$$
\mathcal{G}^{i j k l}(\chi)=\mathcal{G}^{i j k l}\left({ }^{(1)} \chi+{ }^{(2)} \chi\right) .
$$

Which in particular implies that

$$
\mathcal{G}^{i j k l}\left({ }^{(3)} \chi\right)=0 \text {. }
$$

Furthermore, due to the antisymmetry property of ${ }^{(2)} \chi$ one verifies that also

$$
\mathcal{G}^{i j k l}\left({ }^{(2)} \chi\right)=0 \text {. }
$$

Actually, properties (3.39) and (3.40) generalize to

$$
\mathcal{G}^{i j k l}\left({ }^{(2)} \chi+{ }^{(3)} \chi\right)=0
$$

which can be verified, for instance, using computer algebra. Notice that this identity is not trivial since $\mathcal{G}$ depends cubically on the constitutive tensor $\chi$. The identity (3.41) shows that the symmetric piece ${ }^{(1)} \chi$ is indispensable in order to obtain well behaved wave properties: if ${ }^{(1)} \chi=0$, the Fresnel equation is trivially satisfied and thus no lightcone structure could be induced.

\footnotetext{
${ }^{1}$ See http://grtensor.org.
} 
Furthermore, since

$$
\mathcal{G}^{i j k l}\left({ }^{(1)} \chi+{ }^{(2)} \chi\right) \neq \mathcal{G}^{i j k l}\left({ }^{(1)} \chi\right),
$$

the 'skewon' field does influences the Fresnel equation, and therefore, eventually the light cone structure. An example of this general result can be found again in the asymmetric constitutive tensor studied by Nieves and Pal, see section 2.6.1 and references [57, 58].

Actually, after some algebra one finds

$$
\begin{aligned}
\mathcal{G}^{i j k l}\left({ }^{(1)} \chi+{ }^{(2)} \chi\right)= & \mathcal{G}^{i j k l}\left({ }^{(1)} \chi\right) \\
& +\frac{2}{4 !} \hat{\epsilon}_{m n p q} \hat{\epsilon}_{r s t u}{ }^{(1)} \chi^{m n r(i(2)} \chi^{j|p s| k(2)} \chi^{l) q t u} \\
& +\frac{1}{4 !} \hat{\epsilon}_{m n p q} \hat{\epsilon}_{r s t u}{ }^{(2)} \chi^{m n r(i(1)} \chi^{j|p s| k(2)} \chi^{l) q t u} .
\end{aligned}
$$

or, in a (more of less) obvious notation (see the definition (3.28))

$$
\begin{aligned}
\mathcal{G}^{i j k l}(\chi, \chi, \chi)= & \mathcal{G}^{i j k l}\left({ }^{(1)} \chi,{ }^{(1)} \chi,{ }^{(1)} \chi\right)+2 \mathcal{G}^{i j k l}\left({ }^{(1)} \chi,{ }^{(2)} \chi,{ }^{(2)} \chi\right) \\
& +\mathcal{G}^{i j k l}\left({ }^{(2)} \chi,{ }^{(1)} \chi,{ }^{(2)} \chi\right) .
\end{aligned}
$$

The other terms vanish due to the symmetry properties of each irreducible piece.

Take now (3.43) and substitute the parametrization of ${ }^{(2)} \chi$ in terms of $S_{i}{ }^{j}$, see (2.54). After some lengthy but straightforward algebra, one finds that the two last constributions to the right hand side of (3.43) are actually equal, namely

$$
\begin{aligned}
\mathcal{G}^{i j k l}\left({ }^{(1)} \chi,{ }^{(2)} \chi,{ }^{(2)} \chi\right) & =\mathcal{G}^{i j k l}\left({ }^{(2)} \chi,{ }^{(1)} \chi,{ }^{(2)} \chi\right) \\
& =\frac{1}{3}{ }^{(1)} \chi{ }^{m(i|n| j} S_{m}{ }^{k} S_{n}{ }^{l)}
\end{aligned}
$$

Therefore, the final result is

$$
\mathcal{G}^{i j k l}\left({ }^{(1)} \chi+{ }^{(2)} \chi\right)=\mathcal{G}^{i j k l}\left({ }^{(1)} \chi\right)+{ }^{(1)} \chi^{m(i|n| j} S_{m}{ }^{k} S_{n}{ }^{l)} .
$$

A very simple expression indeed.

For the particular case of Nieves and Pal, see (2.54) and (2.84), one finds

$$
\begin{aligned}
\mathcal{G}^{i j k l} q_{i} q_{j} q_{k} q_{l}= & -\sqrt{-g}\left(\frac{\varepsilon_{0}}{\mu_{0}}\right)^{1 / 2} \times \\
& {\left[\frac{\varepsilon_{0}}{\mu_{0}}(q \cdot q)^{2}-(q \cdot q)(v \cdot v)(v \cdot q)^{2}+(v \cdot q)^{4}\right] . }
\end{aligned}
$$


Compare this result with equation (5.7) and reference 12 in [58]. Use $q_{i} \stackrel{*}{=}$ $(\omega,-\vec{k}), v^{i} \stackrel{*}{=}(v, 0,0,0), g_{i j}=\eta_{i j} \stackrel{*}{=}\left(c^{2},-1,-1,-1\right), c^{2}=\frac{1}{\varepsilon_{0} \mu_{0}}$ and obtain

$$
\mathcal{G}^{i j k l} q_{i} q_{j} q_{k} q_{l} \stackrel{*}{=}-\varepsilon_{0}^{3}\left[\left(\omega^{2}-k^{2} c^{2}\right)^{2}+\frac{c^{4}}{\varepsilon_{0}^{2}} k^{2} \omega^{2} v^{4}\right] .
$$

This results agrees with that in [58] (in their $\varepsilon=\mu=1$ case) for $\zeta=\frac{c v^{2}}{\varepsilon_{0}}$.

Nieves and Pal use a different system of units, compare, for instance, their vacuum equations (2.1) and (2.2) with our Maxwell equations (1.1) and (1.2). Their equations can be obtained from our ones by substituting $\mathcal{D}=\mathcal{D}_{\mathrm{N}}, \mathcal{H}=\mathcal{H}_{\mathrm{N}} / c, E=E_{\mathrm{N}} / \varepsilon_{0}, B=B_{\mathrm{N}} /\left(c \varepsilon_{0}\right), \rho=\rho_{\mathrm{N}}, j=j_{\mathrm{N}}$ and $t=t_{\mathrm{N}}$, where the subindex $\mathrm{N}$ refers to the fields in the notation of Nieves and Pal.

Notice that in general, for $\zeta \neq 0$, the Fresnel equation will have complex solutions. This is again a manifestation of the dispersive properties described by the antisymmetric piece ${ }^{(2)} \chi$ of the constitutive tensor.

\subsection{Light rays}

In a riemannian space, light rays are defined as the integral lines of the vector field defined by $V_{\text {riem }}:=g^{i j} q_{j} \partial_{i}$, which is then a null vector. It satisfies $\left.V_{\text {riem }}\right\rfloor q=0$, so that the vector $V_{\text {riem }}$ is orthogonal to the wave front $S$. This definition makes direct use of a metric, it is not a trivial question whether it is possible to define light rays in our more general, pre-metric, framework.

In analogy to the riemannian case, one can try to define light rays as the integral lines of a vector field $V$ which satisfies

$$
V\rfloor q=0 .
$$

Equation (3.50) represents one condition for the 4 independent components of $V$. Therefore, this information is not enough to define light rays uniquely. We know, however, that in the riemannian case the light rays defined by $V_{\text {riem }}$ are also orthogonal to the polarization vector $a$ (such that $f=q \wedge a$ ), i.e. $\left.V_{\text {riem }}\right\rfloor a=0$. The same is true for $b$ with $h=q \wedge b$, i.e. $\left.V_{\text {riem }}\right\rfloor b=0$. These known properties suggest to define the vector $V$ for a given solution of the Maxwell equations as those satisfying

$$
V\rfloor q=0, \quad V\rfloor a=0, \quad V\rfloor b=0 .
$$

The three conditions (3.51) determine then the vector $V$ up to a scalar factor $\left(V^{\prime}=\varphi V\right.$ is also a solution, since (3.51) are homogeneous equations). This 
ambiguity in the definition of $V$ is however irrelevant, since the integral lines of $V$ are independent of that scalar factor ${ }^{2}$.

One can construct immediately a solution of (3.51), since they imply that $V^{i}$ has to be proportional to $\epsilon^{i j k l} q_{j} a_{k} b_{l}$. We therefore define

$$
\mathcal{V}^{i}:=\epsilon^{i j k l} q_{j} a_{k} b_{l}
$$

This quantity is a vector density of weight +1 . Again, the fact that (3.52) does not define a true vector field represents no problem for the definition of light rays as its integral lines. [Alternatively, one could try to 'normalize' $\mathcal{V}$ in order to construct a true vector, for example by dividing $\mathcal{V}$ by the density $|a|^{4}:=\mathcal{G}^{i j k l} a_{i} a_{j} a_{k} a_{l}$, with $|a|$ being a kind of 'norm' of $a$. Obviously, this procedure only makes sense if $|a| \neq 0]$.

Now, using the identities $\epsilon^{i j k l} q_{k} b_{l}=\frac{1}{2} \epsilon^{i j k l} h_{k l}=\frac{1}{2} \chi^{i j k l} f_{k l}=\chi^{i j k l} q_{k} a_{l}$ we can write (3.52) in terms of only $q, a$ and the constitutive tensor, namely

$$
\mathcal{V}^{i}:=\chi^{i j k l} a_{j} a_{k} q_{l}
$$

Notice that, as a consequence of our definitions, $\mathcal{V}$ depends explicitly not only on the wave 1-form $q$ but also on the polarization 1-form $a$. This property is in agreement with the results that, for a general constitutive tensor, different polarization states will propagate in different directions. Birefringence is again a particular example of the above. One also verifies that the axion-like piece of the constitutive tensor drops out from $\mathcal{V}$, so it depends only on ${ }^{(1)} \chi$ and ${ }^{(2)} \chi$.

To gain more physical insight on our definition of light rays, we evaluate the kinematic energy-momentum 3 -form ${ }^{\mathrm{k}} \Sigma_{\alpha}$ for our particular solutions. From its definition (2.125) and using $F \rightarrow f=q \wedge a$ and $H \rightarrow h=q \wedge b$, we find

$$
\begin{aligned}
{ }^{\mathrm{k}} \Sigma_{\alpha} & =\frac{1}{2}\left[q \wedge a \wedge\left(q_{\alpha} b-q b_{\alpha}\right)-q \wedge b \wedge\left(q_{\alpha} a-q a_{\alpha}\right)\right] \\
& =\frac{1}{2}\left[q_{\alpha} q \wedge a \wedge b-q_{\alpha} q \wedge b \wedge a\right] \\
& =q_{\alpha} q \wedge a \wedge b
\end{aligned}
$$

\footnotetext{
${ }^{2}$ If one computes the integral line by $\frac{d x^{i}}{d p}=V^{i}$, then the freedom of choosing the scalar factor corresponds to the freedom of choosing the parameter $p$ along the curve. In other words, an arbitrary scalar factor can always be absorbed by a reparametrization $p^{\prime}=p^{\prime}(p)$.
} 
Compute now the components ${ }^{\mathrm{k}} \mathcal{T}_{\alpha}{ }^{\beta}$ according to (2.126). One finds

$$
\begin{aligned}
\vartheta^{\beta} \wedge{ }^{\mathrm{k}} \Sigma_{\alpha} & =q_{\alpha} \vartheta^{\beta} \wedge q \wedge a \wedge b \\
& =q_{\alpha} q_{\gamma} a_{\delta} b_{\epsilon} \vartheta^{\beta} \wedge \vartheta^{\gamma} \wedge \vartheta^{\delta} \wedge \vartheta^{\epsilon} \\
& =q_{\alpha} q_{\gamma} a_{\delta} b_{\epsilon} \epsilon^{\beta \gamma \delta \epsilon} \hat{\epsilon} \\
& =q_{\alpha} \mathcal{V}^{\beta} \hat{\epsilon} .
\end{aligned}
$$

Therefore,

$$
{ }^{\mathrm{k}} \mathcal{T}_{\alpha}{ }^{\beta}=q_{\alpha} \mathcal{V}^{\beta} .
$$

This result shows that $\mathcal{V}$ determines the direction in which the energymomentum of the wave is transported.

\subsubsection{Fresnel equation for $V$}

Now, since $f=q \wedge a$ and $h=q \wedge b$ we see that the conditions (3.51) imply

$$
V\rfloor h=0, \quad V\rfloor f=0 .
$$

Kiehn [40] uses (3.57) to define $V$ as an 'extremal' vector field. Furthermore, it can be shown that (3.57) are equivalent to (3.51). Take for instance $(3.52 \mathrm{~b})$, then we have

$$
V\rfloor f=V\rfloor(q \wedge a)=(V\rfloor q) a-q(V\rfloor a)=0 .
$$

Suppose now that the scalar $V\rfloor q$ does not vanish, then (3.58) implies that $a$ is proportional to $q$. But this would imply that $f=q \wedge a \equiv 0$. Therefore, nontrivial solutions $(f \neq 0)$ require (3.51a) to hold. Replacing this into (3.58) shows that also (3.51b) must be satisfied. Similarly, starting from (3.52b), the same argument shows that (3.51c) is required.

The equations (3.57) for the vector $V$ are similar to the Hadamard compatibility conditions (3.8) for the wave 1-form $q$. This suggests to apply a similar method as that used in section 3.2 to derive the Fresnel equation for $q$.

First, we solve (3.57b). A general solution can be written as

$$
f=V\rfloor c,
$$

where $c$ is an arbitrary 3 -form. We write the 4 independent components of $c$ as $c_{\alpha \beta \gamma}=: \hat{\epsilon}_{\alpha \beta \gamma \delta} c^{\delta}$. Using this in (3.57a) and (2.63) we find

$$
\left.V\rfloor^{\#}(V\rfloor c\right)=0 .
$$


This equation is analogous to (3.13). As in the case of the wave 1-form $q$, not all the equations in (3.60) are independent, since inner product of $V$ with (3.60) vanishes identically. In analogy to the method used in section 3.2, it is convenient to use a vector frame basis $e_{\alpha}$ and its dual $\vartheta^{\alpha}$, with the special 'gauge' $e_{\hat{0}}=V$. Then, after some algebra one finds that (3.60) is equivalent to

$$
M_{A B} c^{B}=0,
$$

with the $3 \times 3$ matrix $M_{A B}$ defined as

$$
M_{A B}:=\hat{\epsilon}_{\hat{0} A C D} \chi^{C D E F} \hat{\epsilon}_{E F B \hat{0}} .
$$

Again, nontrivial solutions $\left(c^{B} \neq 0\right)$ exist only if $\operatorname{det}\left(M_{A B}\right)=0$, i.e. if and only if

$$
\frac{1}{3 !} \epsilon^{A B C} \epsilon^{D E F} M_{A D} M_{B E} M_{C F}=0 .
$$

Using (3.62) and (3.63), and applying the same method as in section 3.2 to complete two Levi-Civita symbols, we finally find also a quartic equation that now the components of $V$ must satisfy, namely

$$
\mathcal{M}_{i j k l} V^{i} V^{j} V^{k} V^{l}=0
$$

where

$$
\mathcal{M}_{i j k l}:=\frac{1}{4 !} \chi^{m t e f} \hat{\epsilon}_{e f(i \mid h} \hat{\epsilon}_{m|j| n p} \chi^{n p q r} \hat{\epsilon}_{q r|k| s} \hat{\epsilon}_{t \mid l) u v} \chi^{u v h s}
$$

is a totally symmetric tensor density of weight -1 .

Equation (3.64) defines in the space of ray vectors the ray surface, see [45].

\subsubsection{Particular cases}

One can verify after some algebra that in the riemannian case the tensor density $\mathcal{M}_{i j k l}$ reduces to

$$
\mathcal{M}_{i j k l}:=4|g|^{-1 / 2} g_{(i j} g_{k l)} \text {. }
$$

Additionally, one verifies from (3.53) that the vector density $\mathcal{V}$ reduces to

$$
\mathcal{V}^{i}=4|g|^{1 / 2}\left[\left(g^{i k} a_{k}\right)\left(g^{j l} a_{j} q_{l}\right)-\left(g^{i l} q_{l}\right)\left(g^{j k} a_{j} a_{k}\right)\right] .
$$


Since in a riemannian space we additionally have $g^{i j} a_{i} q_{j}=0$, the first term vanishes and the above result shows that $\mathcal{V}^{i} \sim q^{i}:=g^{i j} q_{j}$, as expected.

Notice also that $\mathcal{G}$ is a kind of inverse of $\mathcal{M}$, since they satisfy

$$
\mathcal{G}^{i j k l} \mathcal{M}_{i j k m}=8 \operatorname{sgn}(g) \delta_{m}^{l} \text {. }
$$

It would be interesting to investigate in the future if this property holds also for a general linear medium.

\subsection{Application to propagation of perturba- tions in media with nonlinear constitu- tive relations}

This section is based on the results of Obukhov and myself, see [64].

As a particular case, one can show that the analysis in [60] and references therein about wave propagation in nonlinear theories can be studied with our formalism.

Consider a nonlinear theory in a riemannian space, defined by an action of the form

$$
S=\int \mathcal{L}\left(I_{1}, I_{2}\right) d^{4} x, \quad I_{1}:=F_{i j} F^{i j}, \quad I_{2}:=F_{i j} \tilde{F}^{i j},
$$

with $\mathcal{L}$ a density of weight +1 , in general nonlinear, function of the combinations $F^{i j}:=g^{i k} g^{j l} F_{k l}$ and $\widetilde{F}^{i j}:=\frac{1}{2}|g|^{-1 / 2} \epsilon^{i j k l} F_{k l}$.

From our definition (2.102) we find after some algebra that in this case the effective constitutive tensor is given by

$$
\begin{aligned}
\chi_{\mathrm{eff}}^{i j k l}= & k_{1} g^{i[k} g^{l] j}+k_{2} F^{i j} F^{k l}+k_{3} \tilde{F}^{i j} F^{k l} \\
& +k_{4} F^{i j} \tilde{F}^{k l}+k_{5} \tilde{F}^{i j} \tilde{F}^{k l}+k_{6}|g|^{-1 / 2} \epsilon^{i j k l} .
\end{aligned}
$$

The coefficients $k_{A}, A=1, \ldots, 6$, are function of the electromagnetic fields:

$$
\begin{aligned}
& k_{1}=4 \mathcal{L}_{1}, \quad k_{2}=8 \mathcal{L}_{11}, \quad k_{3}=k_{4}=8 \mathcal{L}_{12}, \\
& k_{5}=8 \mathcal{L}_{22}, \quad k_{6}=2 \mathcal{L}_{2},
\end{aligned}
$$

and $\mathcal{L}_{1}:=\frac{\partial L}{\partial I_{1}}, \mathcal{L}_{12}:=\frac{\partial^{2} L}{\partial I_{1} \partial I_{2}}$, etc. The constitutive tensor $(3.70)$ is symmetric, i.e. the corresponding piece ${ }^{(2)} \chi$ vanishes, since $k_{3}=k_{4}$. We can however 
perform the calculations for the more general case (3.70) for arbitrary factors $k_{A}$. In particular, a direct but rather lengthy calculation of the Fresnel tensor density (3.28) gives

$$
\mathcal{G}^{i j k l}=-\frac{k_{1}}{8}\left(\mathcal{X} g^{(i j} g^{k l)}+2 \mathcal{Y} g^{(i j} t^{k l)}+\mathcal{Z} t^{(i j} t^{k l)}\right)
$$

with

$$
t^{i j}:=F^{i k} F_{k}^{j},
$$

and

$$
\begin{aligned}
\mathcal{X} & :=k_{1}^{2}+\frac{k_{1}}{2}\left(k_{3}+k_{4}\right) I_{2}-k_{1} k_{5} I_{1}+\frac{1}{4}\left(k_{3} k_{4}-k_{2} k_{5}\right) I_{2}^{2}, \\
\mathcal{Y} & :=k_{1}\left(k_{2}+k_{5}\right)+\left(k_{3} k_{4}-k_{2} k_{5}\right) I_{1} \\
\mathcal{Z} & :=4\left(k_{2} k_{5}-k_{3} k_{4}\right) .
\end{aligned}
$$

After inspection of (3.73) one finds that it can be factorized into a product of two second order tensors. One can verify that

$$
\begin{aligned}
\mathcal{G}^{i j k l}= & \frac{-k_{1}}{8 \mathcal{X}}\left[\mathcal{X} g^{(i j}+\left(\mathcal{Y}+\sqrt{\mathcal{Y}^{2}-\mathcal{X Z}}\right) t^{(i j}\right] \\
& \times\left[\mathcal{X} g^{k l)}+\left(\mathcal{Y}-\sqrt{\mathcal{Y}^{2}-\mathcal{X Z}}\right) t^{k l)}\right] \\
= & \frac{-k_{1}}{8 \mathcal{Z}}\left[\left(\mathcal{Y}-\sqrt{\mathcal{Y}^{2}-\mathcal{X \mathcal { Z }}}\right) g^{(i j}+\mathcal{Z} t^{(i j}\right] \\
& \times\left[\left(\mathcal{Y}+\sqrt{\mathcal{Y}^{2}-\mathcal{X \mathcal { Z }}}\right) g^{k l)}+\mathcal{Z} t^{k l)}\right]
\end{aligned}
$$

where the alternative forms are used according to whether $\mathcal{Z}$ or $\mathcal{X}$ vanishes, respectively. If both $\mathcal{Z}$ or $\mathcal{X}$ vanish, then $\mathcal{G}^{i j k l}=-\frac{k_{1}}{4} \mathcal{Y} g^{(i j} t^{k l)}$, as can be seen from (3.73).

Thus, it is a general fact that in any nonlinear electrodynamics model of the type (3.69) the 4th order wave surface always reduces to the two lightcones. These cones are described by the following pair of optical metric tensors:

For $\mathcal{X} \neq 0$, we define

$$
\begin{aligned}
& g_{1}^{i j}:=\mathcal{X} g^{i j}+\left(\mathcal{Y}+\sqrt{\mathcal{Y}^{2}-\mathcal{X \mathcal { Z }}}\right) t^{i j} \\
& g_{2}^{i j}:=\mathcal{X} g^{i j}+\left(\mathcal{Y}-\sqrt{\mathcal{Y}^{2}-\mathcal{X \mathcal { Z }}}\right) t^{i j}
\end{aligned}
$$


For $\mathcal{Z} \neq 0$

$$
\begin{aligned}
& g_{1}^{i j}:=\left(\mathcal{Y}-\sqrt{\mathcal{Y}^{2}-\mathcal{X} \mathcal{Z}}\right) g^{i j}+\mathcal{Z} t^{i j}, \\
& g_{2}^{i j}:=\left(\mathcal{Y}+\sqrt{\mathcal{Y}^{2}-\mathcal{X} \mathcal{Z}}\right) g^{i j}+\mathcal{Z} t^{i j} .
\end{aligned}
$$

And if $\mathcal{X} \neq 0$ and $\mathcal{Z} \neq 0$,

$$
\begin{aligned}
g_{1}^{i j} & :=g^{i j}, \\
g_{2}^{i j} & :=\mathcal{Y} t^{i j} .
\end{aligned}
$$

First, one sees that necessarily $k_{1} \neq 0$ in order to have well behaved wave vectors. Additionally, one can use the definitions (3.75)-(3.77) to compute $\mathcal{Y}^{2}-\mathcal{X} \mathcal{Z}$. After some algebra, one can show that

$$
\begin{aligned}
\mathcal{Y}^{2}-\mathcal{X Z} & =\left[k_{1}\left(k_{2}-k_{5}\right)+\left(k_{3} k_{4}-k_{2} k_{5}\right) I_{1}\right]^{2} \\
& \left.+\left[2 k_{1} k_{3}+\left(k_{3} k_{4}-k_{2} k_{5}\right) I_{2}\right]\left[2 k_{1} k_{4}+\left(k_{3} k_{4}-k_{2} k_{5}\right) I_{2}\right] 3.84\right)
\end{aligned}
$$

This result shows that for $k_{3}=k_{4}$ the quantity $\mathcal{Y}^{2}-\mathcal{X Z}$ is a sum of two squares, and hence non-negative. This means that the corresponding optical metrics $g_{1}$ and $g_{2}$ are always real. See (3.78)-(3.81). For an asymmetric effective constitutive tensor, i.e. $k_{3} \neq k_{4}$, two optical metrics still can be defined, but they are complex in general. For a more complete study, see [64]. 


\section{Chapter 4}

\section{Light cone structure}

In this chapter, we want to study the particular case of our general theory in which a light cone structure is induced. In terms of the Fresnel tensor $\mathcal{G}$, this means that the spacetime/medium is such that

$$
\mathcal{G}^{i j k l}=\mathcal{G}^{(i j} \mathcal{G}^{k l)}=\frac{1}{3}\left(\mathcal{G}^{i j} \mathcal{G}^{k l}+\mathcal{G}^{k j} \mathcal{G}^{i l}+\mathcal{G}^{l j} \mathcal{G}^{i k}\right)
$$

for some symmetric second order tensor density $\mathcal{G}^{i j}$ of weight $+1 / 2$. Then the Fresnel equation, which in general is quartic in the wave-covector, reduces to a second order equation, namely to a lightcone condition:

$$
\mathcal{G}^{i j} q_{i} q_{j}=0 .
$$

Obviously, we are interested in this subcase because in GR (and its particular case SR) the local properties of light propagation in vacuum are determined by the conformal structure of the underlying riemannian geometry. This in particular implies that the wave covectors satisfy a condition (4.2), with $\mathcal{G}^{i j} \sim g^{i j}$. Here $g^{i j}$ denotes the spacetime metric.

We are in particular interested in the following questions: a) What is the most general constitutive tensor leading to a lightcone structure? b) Is it possible to obtain a lightcone structure as a consequence of some metricindependent conditions? In other words, can a lightcone structure be induced/deduced without postulating the existence of a metric from the very beginning? c) If this is the case, how can a conformal metric be explicitly constructed from the underlying constitutive tensor? 


\subsection{Looking for metric-independent conditions}

It is clear that, in order to induce a lightcone structure, additional information or assumptions must the added to the general pre-metric framework, where the constitutive tensor is arbitrary.

We look for these additional conditions. First, we consider the particular case of vacuum in a riemannian space to see if it is possible to find some properties which can be then used as a guide in the pre-metric framework.

We know that the constitutive tensor corresponding to 'vacuum' in a riemannian space, i.e.

$$
\chi_{\{g\}}^{i j k l}=2 \sqrt{\frac{\varepsilon_{0}}{\mu_{0}}} \sqrt{-g} g^{i[k} g^{l] j},
$$

is such that the Fresnel equation reduces to the light cone condition. Note that $\chi_{\{g\}}$ is invariant under conformal transformations $g_{i j} \rightarrow e^{\Psi(x)} g_{i j}$; this takes care that only 9 of the 10 components of the metric can enter $\chi$.

However, since the Fresnel equation is homogeneous, we see that

$$
\chi_{\{g, f\}}^{i j k l}=2 f(x) \sqrt{-g} g^{i[k} g^{l] j},
$$

will also imply the same lightcone structure, for any scalar function $f(x)$. One can use the definition (3.28) of the Fresnel tensor for this particular case and, after some algebra, obtain

$$
\mathcal{G}_{\{g, f\}}^{i j k l}=\operatorname{sgn}(g) f^{3} \sqrt{|g|} g^{(i j} g^{k l)} .
$$

Let us summarize the properties of the constitutive tensor (4.4). Obviously ${ }^{(2)} \chi_{\{g, f\}}=0$, because $\chi_{\{g, f\}}$ is symmetric under the exchange of the first and last pair of indices. Furthermore, ${ }^{(3)} \chi_{\{g, f\}}=0$, i.e., no axion-like term is present. Additionally, one verifies that

$$
\frac{1}{8} \hat{\epsilon}_{i j k l} \chi_{\{g, f\}}^{k l m n} \hat{\epsilon}_{m n p q} \chi_{\{g, f\}}^{p q r s}=-f^{2} 2 \delta_{i}^{[r} \delta_{j}^{s]} .
$$

Notice that in this expression, the metric tensor does not explicitly appear, but the negative sign on the right hand side is due to the its lorentzian signature.

These two properties, can be rewritten in terms of the corresponding linear operator $\kappa$ related to $\chi_{\{g, f\}}=0$, see (2.45). The symmetry property ${ }^{(2)} \chi_{\{g, f\}}=0$ is equivalent to

$$
A \wedge(\kappa B)=(\kappa A) \wedge B
$$


for any 2 -form $A$ and $B$, while the condition (4.6) can be written as

$$
\kappa^{2}=-f^{2} \mathbf{1}
$$

From this particular example from GR, we learn that the riemannian vacuum constitutive tensor satisfies some properties with can be written in a metric-independent way. As we will see in the next sections, an operator satisfying the two conditions above defines a dual operator.

The particular riemannian example can therefore motivate the question whether (4.7) and (4.8) can be taken as the additional conditions to be assumed in order to induce a lightcone structure from the general pre-metric electrodynamic framework, in which the constitutive tensor is arbitrary, and whether these conditions are necessary and/or sufficient to induce a lightcone structure. In fact, it turns out that there exists a relationship between dual operators and conformal metrics.

\subsection{Reciprocity and closure}

Furthermore, the two conditions (4.7) and (4.8) can be motivated already in the pre-metric framework.

From the discussion of section 2.7.2 it is clear that the condition of symmetry ensures that the medium/spacetime will not possess intrinsic dissipative properties. This can then be taken as an additional metric-independent condition. See, however, the discussion in sections 2.6.1 and 3.2.2 about the constitutive tensor of Nieves and Pal, for which ${ }^{(2)} \chi \neq 0$.

The condition of closure can be motivated as follows. An interesting feature of electrodynamics is its electric/magnetic reciprocity property. From the general expression of the kinetic energy-momentum current (2.125), one can be see that the energy-momentum content of the electromagnetic field remains the same if one exchanges excitation $H$ and field strength $F$ in an appropriate way. Consider the transformation

$$
H \rightarrow \zeta F, \quad F \rightarrow-\frac{1}{\zeta} H .
$$

Here $\zeta$ is a pseudoscalar carrying the same physical dimension as the constitutive tensor, i.e. $[\zeta]=[H / F]=q^{2} / h$. Direct inspection of $(2.125)$ shows that the energy-momentum current remains invariant under this transformation, i.e.

$$
{ }^{\mathrm{k}} \Sigma_{\alpha} \rightarrow{ }^{\mathrm{k}} \Sigma_{\alpha}
$$


In terms of the 3-dimensional forms defined in section 2.4, the transformation $H \rightarrow \zeta F$ implies $\mathcal{H} \rightarrow-\zeta E$ and $\mathcal{D} \rightarrow \zeta B$ and $F \rightarrow-\frac{1}{\zeta} H$ implies $E \rightarrow \frac{1}{\zeta} \mathcal{H}$ and $B \rightarrow-\frac{1}{\zeta} \mathcal{D}$. This clearly shows that (4.9) exchange electric and magnetic quantities.

So far, this invariance, which we call electric/magnetic reciprocity ${ }^{1}$, is a property only of the energy-momentum current. If one, as an additional postulate, requires the spacetime/constitutive relation also to be reciprocal, a condition on the constitutive tensor can be found.

Take the spacetime/constitutive relation (2.45) and perform the transformation (4.9) while leaving the constitutive tensor unchanged. One finds

$$
\zeta F_{\alpha \beta}=\frac{1}{4} \hat{\epsilon}_{\alpha \beta \gamma \delta} \chi^{\gamma \delta \epsilon \theta}(-1) \frac{1}{\zeta} H_{\epsilon \theta}
$$

We use again (2.45) to replace the components of the excitation in terms of those of the field strength, and obtain

$$
-\zeta^{2} F_{\alpha \beta}=\frac{1}{16} \hat{\epsilon}_{\alpha \beta \gamma \delta} \chi^{\gamma \delta \epsilon \theta} \hat{\epsilon}_{\epsilon \theta \mu \nu} \chi^{\mu \nu \lambda \rho} F_{\lambda \rho} .
$$

This expression leads us to a condition for the constitutive tensor, namely to

$$
-\zeta^{2} \delta_{\alpha}^{[\lambda} \delta_{\beta}^{\rho]}=\frac{1}{16} \hat{\epsilon}_{\alpha \beta \gamma \delta} \chi^{\gamma \delta \epsilon \theta} \hat{\epsilon}_{\epsilon \theta \mu \nu} \chi^{\mu \nu \lambda \rho}
$$

Define now the dimensionless tensor

$$
\stackrel{o}{\chi}^{\alpha \beta \gamma \delta}:=\frac{1}{\zeta} \chi^{\alpha \beta \gamma \delta}
$$

with

$$
\zeta^{2}:=-\frac{1}{96}\left(\hat{\epsilon}_{i j m n} \chi^{m n p q}\right)\left(\hat{\epsilon}_{p q r s} \chi^{r s i j}\right)
$$

Then (4.13) can be rewritten as

$$
\frac{1}{8} \hat{\epsilon}_{\alpha \beta \gamma \delta} \stackrel{o}{\chi}^{\gamma \delta \epsilon \theta} \hat{\epsilon}_{\epsilon \theta \mu \nu} \stackrel{o}{\chi}^{\mu \nu \lambda \rho}=-2 \delta_{\alpha}^{[\lambda} \delta_{\beta}^{\rho]} .
$$

\footnotetext{
${ }^{1}$ to distinguish it from the usual metric-dependent duality transformation $F_{i j} \rightarrow \widetilde{F}_{i j}:=$ $\frac{1}{2} \sqrt{-g} \hat{\epsilon}_{i j k l} g^{k m} g^{l n} F_{m n}$, under which the vacuum $(J=0)$ Maxwell equations in a riemannian space are invariant.
} 
It is convenient to define

$$
J_{\alpha \beta}^{\gamma \delta}:=\frac{1}{2} \hat{\epsilon}_{\alpha \beta \eta \sigma} \stackrel{o}{\chi}^{\eta \sigma \gamma \delta}
$$

Then the closure relation (4.16) reads

$$
\frac{1}{2} J_{\alpha \beta}^{\eta \sigma} J_{\eta \sigma}{ }^{\gamma \delta}=-2 \delta_{\alpha}^{[\lambda} \delta_{\beta}^{\rho]}
$$

or, in the corresponding notation in terms of $6 \times 6$ matrices,

$$
J^{2}=-1_{6}
$$

We call this condition on the constitutive tensor the closure relation. Notice that the negative sign on $(4.9 \mathrm{~b})$ and therefore the one on the right hand side of (4.18), is a consequence of the negative relative sign of the two terms entering in the energy-momentum current, see (2.45). Below we will see that this negative sign will be responsible of the lorentzian signature of the induced metric.

Mathematically, this means that the operator $J$ represents an almost complex structure on the space of 2 -forms.

\subsection{Dual operators and metrics}

A linear operator $J: \Lambda^{2}(X) \rightarrow \Lambda^{2}(X)$ acting on 2-forms is said to be a dual operator defining a complex structure if it satisfies the properties of symmetry,

$$
A \wedge(J B)=(J A) \wedge B
$$

for any 2 -form $A$ and $B$, and if it is closed such that

$$
J^{2}=-1 \text {. }
$$

Many people have studied this relationship, both as a useful tool in GR $[6,7]$ and as a method which could allow to consider the metric of spacetime as a secondary quantity, constructed from some other fields, see for instance [8]. Clearly, the dimensionless part of the riemannian constitutive tensor $f^{-1} \chi_{\{g, f\}}^{i j k l}$, defines a dual operator, which is just the Hodge dual of the metric $g$.

In the context of electrodynamics, it seems that Peres [68] was the first to try to reconstruct the conformally invariant part of the spacetime metric 
from the excitation $H$ and field strength $F$. In his approach, however, the metric is assumed to exist such that the relation between $H$ and $F$ is just the same that the one of vacuum in a riemannian space. In our notation, this condition is equivalent to postulate that the operator $\kappa$ is a dual operator in the sense defined above and that it also equals the Hodge dual of some metric, to be determined. In this sense, the approach of Peres can be considered as a sort of 'inverse problem'. Contrary to define the operator $\kappa$ as the dual of the metric tensor, as is done in GR, Peres tried to determine a metric such that its Hodge dual operator coincides with $\kappa$. To that goal, it is assumed that the operator $\kappa$ satisfies the conditions equivalent, in our notation, to symmetry and closure, as a Hodge dual operator does. However, as we will discuss, see below, Peres did not succeed in deriving the conformally metric, since his result is not unique.

Toupin [92] and Schönberg [77] also studied how a conformal metric structure is induced and in particular how the conformally invariant part of the metric can be deduced from the spacetime/constitutive relation under the assumption of symmetry and closure. They seem to be the first who were able to show that a conformal metric structure is actually implied as a consequence of symmetry and closure. Brans $[6,7]$ also recognized that, within general relativity, it is possible to recover the metric from its Hodge dual operator. These structures were subsequently discussed by numerous people, by 't Hooft [91], Harnett [22], and Obukhov \& Tertychniy [61], amongst others, see also the references given there.

From these studies, it seems clear by now that there is a one-to-one correspondence between linear operators (of the kind of $\kappa$ ) satisfying symmetry and closure, and conformal metrics. If a metric is available, one can immediately construct the corresponding Hodge dual operator, which, for a metric with lorentzian signature, will satisfy (4.20) and (4.21). On the other hand, the contrary is also valid. If an operator $J$ satisfying (4.20) and (4.21) is available, then a conformal metric structure is induced, such that the operator $J$ can be written as a Hodge dual operator. Furthermore, the metric components can be (re-)constructed using the so-called Schönberg-Urbantke formula. Schönberg seems to be the first to derive this formula in the context of electrodynamics. This formula was also found by Urbantke [94, 95], but in a different context, namely $S U(2)$ Yang-Mills theories. We will discuss some derivations of this important formula below.

Finally, by using Schönberg-Urbantke's formula in the context of linear pre-metric electrodynamics, Obukhov and Hehl [63] presented an explicit 
construction of the conformally invariant part of the metric tensor in terms of quantities parametrizing a constitutive tensor satisfying symmetry and closure.

After discussing these developments, an alternative procedure to deduce the conformal metric and its lightcone structure will be presented. It relies on the general results about the Fresnel equation governing the local properties of electromagnetic waves. This alternative approach is therefore much more suitable to understand the physics underlying the emergence of the conformal structure of spacetime, since it directly involves the properties of the propagation of waves. Finally, this approach also allows to study what would be the effects of dropping or modifying the assumptions of symmetry and closure on the lightcone structure. In particular, we will explore the consequences of a possible asymmetric spacetime/constitutive relation would have on the lightcone, but maintaining the assumption of closure.

\subsubsection{Peres's early work}

In [68] Peres already explores the idea of deriving the metric tensor from the electromagnetic fields $H$ and $F$. Here we discuss the attempt of Peres to reconstruct the conformal metric.

Assume that a metric tensor is defined on the manifold such that the closure relation takes its usual riemannian form, namely

$$
H=\lambda^{*} F,
$$

with $\lambda:=\sqrt{\varepsilon_{0} / \mu_{0}}$ and ${ }^{*}$ is the Hodge dual operator of the metric $g$. This is equivalent to assume that a metric tensor $g$ exists such that the constitutive tensor can be written in the form (4.3).

The problem is then basically to determine the conformal metric components $g^{i j}$ in terms of $H$ and $F$ such that (4.22) is satisfied. A necessary condition for $g$ to satisfy (4.22) can be found by computing the 4 -form $H \wedge H$. Using (4.22) one finds

$$
H \wedge H=\lambda^{2 *} F \wedge^{*} F=-\lambda^{2} F \wedge F .
$$

Therefore, it is necessary that

$$
H \wedge H=-\lambda^{2} F \wedge F
$$

for every field configuration $F$ and $H$. The negative sign in this equation is a consequence of the lorentzian signature of the metric $g$. Equation (4.24) is 
Peres's version of the closure relation (4.18). Since it must be satisfied for any field configuration, (4.24) is equivalent to

$$
\frac{1}{4} \hat{\epsilon}_{i j k l} \chi_{\{g\}}^{i j m n} \chi_{\{g\}}^{k l p q}=-\epsilon^{m n p q}
$$

which is equivalent to the closure relation $(4.18)$, since ${ }^{(2)} \chi_{\{g\}}=0$, as it is implicitly assumed in (4.22).

In order to reconstruct the metric, Peres uses the following method. Consider now the eigenvalue problem

$$
V\rfloor M=0,
$$

with

$$
M:=H+\lambda k F
$$

and $k$ being a dimensionless scalar and $V$ a vector. This eigenvalue problem defines the vectors $V$ in terms of $H$ and $F$.

The condition for nonvanishing solutions $V$ to exist can be found as follows. The condition (4.26) implies

$$
V\rfloor(M \wedge M)=0,
$$

and since $M \wedge M$ is a 4 -form this later condition necessarely implies $M \wedge M=$ 0 . Using the definition (4.27) and the property (4.24) we then obtain

$$
\begin{aligned}
M \wedge M & =H \wedge H+2 \lambda k H \wedge F+\lambda^{2} k^{2} F \wedge F \\
& =-\lambda^{2} F \wedge F+2 \lambda k H \wedge F+\lambda^{2} k^{2} F \wedge F .
\end{aligned}
$$

If we write the 4 -forms $H \wedge F$ and $F \wedge F$ in terms of the invariants $I_{1}$ and $I_{2}$ as

$$
H \wedge F=: \lambda I_{1} \hat{\epsilon}, \quad F \wedge F=: I_{2} \hat{\epsilon}
$$

then we find that the eigenvalue equation (4.26) allows nonvanishing solutions if and only if the scalar $k$ satisfy the quadratic equation

$$
k^{2} I_{2}+2 k I_{1}-I_{2}=0 .
$$

Notice that $I_{1}$ and $I_{2}$ are defined such that they carry the same dimension: $\left[I_{1}\right]=\left[I_{2}\right]=h^{2} / q^{2}$. 
The solutions of (4.31) are

$$
k^{ \pm}=-\frac{1}{I_{2}}\left[I_{1} \pm \sqrt{I_{1}^{2}+I_{2}^{2}}\right],
$$

which in particular imply that $k^{+} k^{-}=-1$, so that one solution is necessarily positive and the other negative.

Using a particular coordinate system, Peres finds solutions of the equation (4.26) for the vector $V$. The particular coordinate system is chosen such that the Lorentzian metric (that Peres is looking for) assumes its minkowskian form, i.e.,

$$
g_{i j} \stackrel{*}{=} \eta_{i j} \stackrel{*}{=} \operatorname{diag}(+1,-1,-1,-1) .
$$

Additionally, a local Lorentz transformation is performed such that the electromagnetic field strength adopts the simple form given by $E_{a}=(E, 0,0)$ and $B^{a}=(B, 0,0)$. It is also assumed that $E>0$ and $B>0$. This means that

$$
F \stackrel{*}{=}-E d x^{0} \wedge d x^{1}+B d x^{2} \wedge d x^{3} \text {. }
$$

As a consequence of (4.22) and (4.33) one finds the excitation to be

$$
H \stackrel{*}{=} \lambda\left[B d x^{0} \wedge d x^{1}+E d x^{2} \wedge d x^{3}\right] .
$$

In this particular coordinate system, the invariants defined in (4.30) take the form

$$
I_{1} \stackrel{*}{=} B^{2}-E^{2}, \quad I_{2} \stackrel{*}{=}-2 E B .
$$

This implies, see (4.32), that $k$ can assume the values

$$
k^{+} \stackrel{*}{=} \frac{B}{E}, \quad k^{-\stackrel{*}{=}}-\frac{E}{B} \text {. }
$$

Additionally, if one writes the vector $V$ as $V \stackrel{*}{=} V^{0} \partial_{0}+V^{1} \partial_{1}+V^{2} \partial_{2}+V^{3} \partial_{3}$ then the eigenvalue equation (4.26) reduces to

$$
V\rfloor M=\lambda(B-k E)\left(v^{0} d x^{1}-v^{1} d x^{0}\right)+\lambda(E+k B)\left(v^{2} d x^{3}-v^{3} d x^{2}\right) .
$$

With this information, one sees that for $k=k^{+}$necessarely $V^{2}=V^{3}=0$. This means that the vectors $V$ which are solutions of the above equations for $k=k^{+}$are linear combinations of $V_{+A}, A, B, \ldots=1,2$, with

$$
V_{+1}: \stackrel{*}{=}(E B)^{1 / 4} \partial_{0}, \quad V_{+2}: \stackrel{*}{=}(E B)^{1 / 4} \partial_{1} .
$$


For $k=k^{-}$(4.38) implies $V^{0}=V^{1}=0$ and therefore the corresponding solutions are linear combinations of $V_{-A}$ with

$$
V_{-1}: \stackrel{*}{=}(E B)^{1 / 4} \partial_{2}, \quad V_{-2}: \stackrel{*}{=}(E B)^{1 / 4} \partial_{3} .
$$

The factors $(E B)^{1 / 4}$ are introduced for later convenience. It can then be verified by direct computation that these vectors satisfy the following properties:

$$
\begin{aligned}
& \left.\left.V_{+A}\right\rfloor\left(V_{-B}\right\rfloor F\right)=0, \\
& \left.\left.V_{+A}\right\rfloor\left(V_{+B}\right\rfloor F\right)=-\frac{I_{2}}{2}\left|k^{-}\right|^{1 / 2} \epsilon_{A B}, \\
& \left.\left.V_{-A}\right\rfloor\left(V_{-B}\right\rfloor F\right)=\frac{I_{2}}{2}\left|k^{+}\right|^{1 / 2} \epsilon_{A B},
\end{aligned}
$$

with $\epsilon_{A B}=\epsilon_{[A B]}$ and $\epsilon_{12}:=1$.

Furthermore, the excitation and field strength can be written as

$$
\begin{aligned}
& \left.\left.\left.\left.F=-\frac{1}{2} \epsilon^{A B}\left[\left|k^{+}\right|^{1 / 2}\left(V_{+A}\right\rfloor V_{+B}\right\rfloor \hat{\epsilon}\right)-\left|k^{-}\right|^{1 / 2}\left(V_{-A}\right\rfloor V_{-B}\right\rfloor \hat{\epsilon}\right)\right] \\
& \left.\left.\left.\left.H=-\frac{\lambda}{2} \epsilon^{A B}\left[\left|k^{-}\right|^{1 / 2}\left(V_{+A}\right\rfloor V_{+B}\right\rfloor \hat{\epsilon}\right)+\left|k^{+}\right|^{1 / 2}\left(V_{-A}\right\rfloor V_{-B}\right\rfloor \hat{\epsilon}\right)\right] .
\end{aligned}
$$

Compute, for instance, the field strength $F$ :

$$
\begin{aligned}
F & \left.\left.\left.\left.\stackrel{*}{=}-\frac{1}{2} \epsilon^{A B}\left[\left|k^{+}\right|^{1 / 2}\left(V_{+A}\right\rfloor V_{+B}\right\rfloor \hat{\epsilon}\right)-\left|k^{-}\right|^{1 / 2}\left(V_{-A}\right\rfloor V_{-B}\right\rfloor \hat{\epsilon}\right)\right] \\
& \left.\left.\left.\left.\stackrel{*}{=}-\epsilon^{12}\left[\left|k^{+}\right|^{1 / 2}\left(V_{+1}\right\rfloor V_{+2}\right\rfloor \hat{\epsilon}\right)-\left|k^{-}\right|^{1 / 2}\left(V_{-1}\right\rfloor V_{-2}\right\rfloor \hat{\epsilon}\right)\right] \\
& \left.\left.\left.\left.\stackrel{*}{=}-\left[\left(\frac{B}{E}\right)^{1 / 2}(E B)^{1 / 2}\left(\partial_{0}\right\rfloor \partial_{1}\right\rfloor \hat{\epsilon}\right)-\left(\frac{E}{B}\right)^{1 / 2}(E B)^{1 / 2}\left(\partial_{2}\right\rfloor \partial_{3}\right\rfloor \hat{\epsilon}\right)\right] \\
& \stackrel{*}{=} B d x^{2} \wedge d x^{3}-E d x^{0} \wedge d x^{1}
\end{aligned}
$$

which agrees with (4.34).

Although (4.41)-(4.45) were derived in a particular coordinate system, they are covariant equations and therefore are valid in any coordinate system. In other words, when solving the eigenvalue problem (4.26) one can always find a basis of solutions $V_{+A}$ and $V_{-A}$ corresponding to $k^{+}$and $k^{-}$(given by (4.32)) such that (4.41)-(4.42) are fulfilled. 
Finally, in Peres's approach the metric tensor is also constructed bilinearly in the covectors $V_{ \pm A}$. We define the inverse metric $g^{i j}$ by

$$
g^{i j}=S\left(Q_{+}^{A B} V_{+A}^{i} V_{+B}^{j}+Q_{-}^{A B} V_{-A}^{i} V_{-B}^{j}\right),
$$

where $Q_{ \pm}^{A B}=Q_{ \pm}^{B A}$ are two symmetric $2 \times 2$ matrices. As a consequence, the determinant is found to be given by

$$
|g|^{-1 / 2}=-\frac{I_{2}}{2} S^{2}\left|Q_{+} Q_{-}\right|^{1 / 2}
$$

where $Q_{ \pm}$denote the respective determinants, i.e., $Q_{ \pm}:=\operatorname{det}\left(Q_{ \pm}^{A B}\right)$.

With the ansatz (4.47) one can look for the conditions for the matrices $Q_{ \pm}^{A B}$ to be satisfied so that the metric (4.47) fulfills (4.22). The action of the Hodge dual operator of $g$ on $F$ can be written as

$$
{ }^{*} F=\frac{1}{4}|g|^{1 / 2} \hat{\epsilon}_{i j k l} g^{k m} g^{l n} F_{m n} d x^{i} \wedge d x^{j} .
$$

Rewriting the right hand side of (4.49) using (4.47), (4.48), and the properties (4.41)-(4.43), one finds

$$
\begin{aligned}
&{ }^{*} F= \frac{1}{4}|g|^{1 / 2} \hat{\epsilon}_{i j k l} g^{k m} g^{l n} F_{m n} d x^{i} \wedge d x^{j} \\
&=-\frac{1}{2 I_{2}\left|Q_{+} Q_{-}\right|{ }^{1 / 2}} \hat{\epsilon}_{i j k l}\left(Q_{+}^{A B} V_{+A}^{k} V_{+B}^{m}+Q_{-}^{C D} V_{-C}^{k} V_{-D}^{m}\right) \\
& \times\left(Q_{+}^{C D} V_{+C}^{l} V_{+D}^{n}+Q_{-}^{A B} V_{-A}^{l} V_{-B}^{n}\right) F_{m n} d x^{i} \wedge d x^{j} \\
&=-\frac{1}{2 I_{2}\left|Q_{+} Q_{-}\right|{ }^{1 / 2}} \hat{\epsilon}_{i j k l}\left[Q_{+}^{A B} Q_{+}^{C D} V_{+A}^{k} V_{+C}^{l}\left(V_{+B}^{m} V_{+D}^{n} F_{m n}\right)\right. \\
&\left.\quad+Q_{-}^{A B} Q_{-}^{C D} V_{-A}^{k} V_{-C}^{l}\left(V_{-B}^{m} V_{-D}^{n} F_{m n}\right)\right] d x^{i} \wedge d x^{j} \\
&=-\frac{\epsilon_{B D}}{4\left|Q_{+} Q_{-}\right|{ }^{1 / 2}} \hat{\epsilon}_{i j k l}\left[Q_{+}^{A B} Q_{+}^{C D} V_{+A}^{k} V_{+C}^{l}\left|k^{-}\right|^{1 / 2}\right. \\
&\left.\quad-Q_{-}^{A B} Q_{-}^{C D} V_{-A}^{k} V_{-C}^{l}\left|k^{+}\right|^{1 / 2}\right] d x^{i} \wedge d x^{j} \\
&=-\frac{\epsilon^{A C}}{4\left|Q_{+} Q_{-}\right| 1 / 2} \hat{\epsilon}_{i j k l}\left[Q_{+}\left|k^{-}\right|^{1 / 2} V_{+A}^{k} V_{+C}^{l}\right. \\
& \quad\left.Q_{-}\left|k^{+}\right|^{1 / 2} V_{-A}^{k} V_{-C}^{l}\right] d x^{i} \wedge d x^{j} .
\end{aligned}
$$

We now use the identity

$$
\left.\left.\hat{\epsilon}_{i j k l} V_{ \pm A}^{k} V_{ \pm C}^{l} d x^{i} \wedge d x^{j}=-2\left(V_{ \pm A}\right\rfloor V_{ \pm C}\right\rfloor \hat{\epsilon}\right)
$$


and rewrite $(4.50)$ as

$$
\begin{array}{r}
\left.{ }^{*} F=\frac{\epsilon^{A C}}{2\left|Q_{+} Q_{-}\right|^{1 / 2}}\left[Q_{+}\left|k^{-}\right|^{1 / 2}\left(V_{+A}\right\rfloor V_{+C}\right\rfloor \hat{\epsilon}\right) \\
\left.\left.\left.\quad-Q_{-}\left|k^{+}\right|^{1 / 2}\left(V_{-A}\right\rfloor V_{-C}\right\rfloor \hat{\epsilon}\right)\right] d x^{i} \wedge d x^{j} .
\end{array}
$$

With this result and the expression (4.45) for the excitation we find that the conditions on the $Q$-matrices for (4.47) to fulfill (4.22) are:

$$
\frac{Q_{+}}{\left|Q_{+} Q_{-}\right|^{1 / 2}}=-1, \quad \frac{Q_{-}}{\left|Q_{+} Q_{-}\right|^{1 / 2}}=1,
$$

which is fulfilled provided

$$
Q_{-}=-Q_{+}, \quad Q_{-}>0 .
$$

Furthermore, due to the arbitrary factor $S$ in the ansatz (4.47), one can choose

$$
Q_{-}=-Q_{+}=1
$$

without restricting the generality of the metric. For the special coordinate system in which (4.39) and (4.40) hold, the choice

$$
Q_{ \pm}^{A B}=\left(\begin{array}{cc} 
\pm 1 & 0 \\
0 & -1
\end{array}\right)
$$

leads to

$$
g^{i j} \stackrel{*}{=} S(E B)^{1 / 2} \eta^{i j},
$$

i.e., to a conformal Minkowski metric.

Summing up, Peres re-constructs the spacetime metric, which is assumed to exist, in terms of four vectors $V_{ \pm A}, A, B, \ldots=1,2$, satisfying

$$
\begin{aligned}
& \left.\left.V_{+A}\right\rfloor\left(V_{-B}\right\rfloor F\right)=0 . \\
& \left.\left.V_{+A}\right\rfloor\left(V_{+B}\right\rfloor F\right)=-\frac{I_{2}}{2}\left|k^{-}\right|^{1 / 2} \epsilon_{A B}, \\
& \left.\left.V_{-A}\right\rfloor\left(V_{-B}\right\rfloor F\right)=\frac{I_{2}}{2}\left|k^{+}\right|^{1 / 2} \epsilon_{A B} .
\end{aligned}
$$


Then the metric is given by

$$
g^{-1}=S\left(Q_{+}^{A B} V_{+A} \otimes V_{+B}+Q_{-}^{A B} V_{-A} \otimes V_{-B}\right)
$$

with

$$
Q_{-}=-Q_{+}=1
$$

However, this prescription for constructing the metric is not unique. Consider that (4.58)-(4.60) are solved for some particular vectors $V_{ \pm A}$ in a given coordinate system. Then the only condition on the symmetric matrices $Q_{ \pm}^{A B}$ is $Q_{-}=-Q_{+}=1$. This leaves 2 independent functions for each matrix $Q$. Additionally, the function $S$, related to the conformal invariance of (4.22), is also arbitrary. Therefore, the metric constructed by Peres contains 5 arbitrary functions. In the approach of Peres, therefore, not all the information about the lightcone structure is extracted from the fields $F$ and $H$. It is also unclear which additional conditions must be imposed to obtain a unique class of conformal metrics.

There seems to be no clear relationship between the quantities involved in the work of Peres (for instance, the vectors $V_{ \pm I}$ ) with the objects introduced by Schönberg and Harnett, see below.

\subsubsection{Schönberg-Urbantke formula}

Here we discuss the the successful derivation of of the conformal metric by Schönberg and the related work of Harnett.

\section{Schönberg's derivation}

In his work [77] Schönberg studied a different derivation of the metric from electromagnetism. Additionally, the analysis in [77] helps to better understand the group theoretical aspects of the emergence of a lorentzian metric structure, and therefore of the Lorentz group.

As we already saw before, the electromagnetic field strength $F$, with its six independent components, can be described as a vector in a 6-dimensional real vector space. We call this space $S_{6}$. One can map a basis of the space of 2 -form, $d x^{i_{1}} \wedge d x^{i_{2}}$ to a basis $b^{I}$ of $S_{6}$ by means of the rule $\left[i_{1} i_{2}\right] \rightarrow I=$ $01,02,03,23,31,12$. Then one can write a vector $A \in S_{6}$ as $A=A_{I} b^{I}$. 
An important role is played by an inner product $\epsilon$ on $S_{6}$ induced by the 4-dimensional Levi-Civita symbol $\epsilon^{i j k l}$. Given two vectors $A$ and $B$ of $S_{6}$, one can define their product as

$$
\epsilon(A, B):=\epsilon^{I J} A_{I} B_{J}
$$

In 4-dimensional notation, this is equivalent to

$$
\epsilon(A, B) \hat{\epsilon}:=A \wedge B
$$

or, in components,

$$
\epsilon(A, B)=\frac{1}{4} \epsilon^{i j k l} A_{i j} B_{k l} .
$$

Thus, the Levi-Civita symbol acts as a metric in the 6-dimensional space $S_{6}$. Notice however that $\epsilon(A, B)$ is a 4 -dimensional density of weight +1 . Since the eigenvalues of $\epsilon^{I J}$ are +1 and -1 , each with multiplicity 3 (see $(2.73 \mathrm{~b}))$, the signature of $\epsilon^{I J}$ is $(+1,+1,+1,-1,-1,-1)$. This immediately shows that the 6-dimensional space $S_{6}$ naturally contains a $S O(3,3)$ group structure. Transformation of $S_{6}$-vectors under the action of the $S O(3,3)$ group leaves the product (4.63) invariant. It is important to emphasize that this group structure is always present, independent of any metric, affine, or whatever additional structure on the 4-dimensional manifold.

Using this product, one can express the assumption of symmetry of the constitutive tensor, i.e. ${ }^{(2)} \chi=0$ as

$$
\epsilon(A, J B)=\epsilon(J A, B)
$$

for all $A, B \in S_{6}$, because

$$
\epsilon(A, J B)=\epsilon^{I J} A_{I} J_{J}{ }^{K} B_{K}=\chi^{I K} A_{I} B_{K}
$$

since $\chi^{I K}=\epsilon^{I J} J_{J}^{K}$ and

$$
\epsilon(J A, B)=\epsilon^{I J} J_{I}^{K} A_{K} B_{J}=\chi^{I K} A_{K} B_{J}=\chi^{K I} A_{I} B_{K},
$$

so that (4.66) requires symmetry of $\chi$, i.e., $\chi^{I J}=\chi^{J I}$.

Furthermore, the additional condition of closure of the operator $J$, i.e.,

$$
J^{2}=-1
$$

is assumed to hold. 
In the 6-dimensional real space $S_{6}$, the introduction of the linear operator $J$ satisfying (4.66) and (4.69) induces a complex structure. In order to be able to discuss this structure and in particular the eigenvectors of the operator $J$, consider the complex extension of $S_{6}$, i.e. the vector space of the complex 2forms $S_{6}(C)$. The negative sign on the right hand side of (4.69) implies that the eigenvalues of $J$ are $\pm i$, and since $J$ is a real operator each eigenvalue must have multiplicity 3 . This means that the corresponding self-dual and antiselfdual subspaces spanned by vectors with eigenvalues $+i$ and $-i$ are both 3dimensional. We denote these subspaces as $S_{3}^{+}(C)$ and $S_{3}^{-}(C)$ respectively. The vectors of $S_{3}^{ \pm}(C)$ will be denoted as $A^{ \pm}$and satisfy

$$
J A^{ \pm}= \pm i A^{ \pm} .
$$

Take any $A^{ \pm} \in S_{3}^{ \pm}(C)$ and write it as $A^{ \pm}=A+i A^{\prime}$ with $A$ and $A^{\prime}$ real 6-dimensional vectors (i.e. $A, A^{\prime} \in S_{6}$ ). Then from (4.70) one finds that

$$
J\left(A+i A^{\prime}\right)= \pm i\left(A+i A^{\prime}\right),
$$

with implies

$$
J A=\mp A^{\prime}, \quad J A^{\prime}= \pm A .
$$

Using (4.72) one can rewrite every element $S^{ \pm}$of $S_{3}^{ \pm}$, in terms of only the real $A \in S_{6}$ and the operator $J$, as

$$
A^{ \pm}=A \mp i J A .
$$

The spaces $S_{6}^{+}(C)$ and $S_{6}^{-}(C)$ are orthogonal with respect to the product $\epsilon$. Take any $A^{+} \in S_{6}^{+}(C)$ and $B^{-} \in S_{6}^{-}(C)$. Write them in the form (4.73), i.e. $A^{+}=A-i J A$ and $B^{-}=B+i J B$, and compute their $\epsilon$-product. Using the symmetry and closure properties (4.66) and (4.69) one finds

$$
\begin{aligned}
\epsilon\left(A^{+}, B^{-}\right) & =\epsilon(A-i J A, B+i J B) \\
& =\epsilon(A, B)+i \epsilon(A, J B)-i \epsilon(J A, B)+\epsilon(J A, J B) \\
& =\epsilon(A, B)+i \epsilon(A, J B)-i \epsilon(A, J B)+\epsilon\left(A, J^{2} B\right) \\
& =\epsilon(A, B)+\epsilon(A,-B) \\
& =0 .
\end{aligned}
$$

Consider a real operator $M$ acting on the real space $S_{6}$. If $A \in S_{6}$ then also $M A \in S_{6}$. However, the self-dual element $(M A)^{+}$of $S_{3}^{+}$will in general not be the result of the application of the linear operator $M$ on $A^{+}$, since

$$
(M A)^{+}=(M A)-i J(M A) \neq M(A-i J A)=M\left(A^{+}\right),
$$


unless the operators $M$ and $J$ commute, i.e. $[M, J]=0$. In other words, not every linear operator on $S_{6}$ is a linear operator in $S_{3}^{+}$. In particular, not every element of the group $O(3,3)$ which leaves $\epsilon$ invariant defines a linear operator in the self-dual space $S_{3}^{+}$.

The corresponding subgroup of $O(3,3)$ which commutes with $J$, i.e. the subgroup form by those operators $N$ such that

$$
\epsilon(N A, N B)=\epsilon(A, B), \quad[N, J]=0,
$$

can be shown to be isomorphic to the Lorentz group. To prove this, consider the product $\epsilon$ restricted to the self-dual subspace $S_{3}^{+}$, which we denote as $\epsilon^{+}$, and is defined by

$$
\epsilon^{+}\left(A^{+}, B^{+}\right):=\epsilon\left(A^{+}, B^{+}\right) .
$$

Consider operators $N$ satisfying (4.76) which are therefore also linear operators of $S_{3}^{+}$. It is clear that they will also leave invariant the 3 -dimensional product $\epsilon^{+}$, since in this case

$$
\begin{aligned}
\epsilon^{+}\left((N A)^{+},(N B)^{+}\right) & =\epsilon^{+}\left(N A^{+}, N B^{+}\right) \\
& =\epsilon\left(N A^{+}, N B^{+}\right) \\
& =\epsilon\left(A^{+}, B^{+}\right) \\
& =\epsilon^{+}\left(A^{+}, B^{+}\right) .
\end{aligned}
$$

This shows that the subgroup of $O(3,3)$ commuting with $J$ forms the invariance group of the 3-dimensional metric induced by $\epsilon^{+}$in $S_{3}^{+}$. But since $S_{3}^{+}$is a complex linear space, the corresponding invariance group of $\epsilon^{+}$is clearly $S O(3, C)$, which is isomorphic to the Lorentz group $S O(3,1)$. In other words, an operator $J$ satisfying symmetry (4.66) and closure (4.69) induces a $S O(3, C) \approx S O(3,1)$ group structure, which manifest itself as the symmetry group of the natural metric structure on the self-dual space of $J$. Alternatively, the same arguments can be repeated considering the anti-selfdual space $S_{3}^{+}$.

We consider now how to reconstruct the metric components of the corresponding induced lorentzian metric. Consider a basis for each subspace. We denote the basis of $S_{3}^{+}$by $S_{+}^{(a)}, a, b, \ldots=1,2,3$. Then the complex conjugate $S_{-}^{(a)}:=\left(S_{+}^{(a)}\right)^{*}$ is a basis of $S_{3}^{-}$, see (4.70). Since $S_{6}(C)=S_{3}^{+} \oplus S_{3}^{-}$, the six $S_{6}$-vectors $\left\{S_{+}^{(a)}, S_{-}^{(a)}\right\}$ form a basis of $S_{6}(C)$. Then the orthogonality 
property (4.74) implies that the components of the metric $\epsilon$ of $S_{6}(C)$ in the basis $\left\{S_{+}^{(a)}, S_{-}^{(a)}\right\}$ form a block-diagonal matrix, since $\epsilon\left(S_{+}^{(a)}, S_{-}^{(b)}\right)=0$.

One can use the exterior product of vectors in $S_{6}(C)$, which we denote by $\triangle$, and determine a volume element of $S_{3}^{+}$as

$$
W_{+}:=S_{+}^{(1)} \triangle S_{+}^{(2)} \triangle S_{+}^{(3)}=\frac{1}{3 !} \hat{\epsilon}_{a b c} S_{+}^{(a)} \triangle S_{+}^{(b)} \triangle S_{+}^{(c)} \text {. }
$$

Then $W_{+}$is a 3 -form on $S_{6}$, i.e. $W_{+} \in \Lambda^{3}\left(S_{6}\right)$. Since $S_{3}^{+}$is 3-dimensional, $W_{+}$is, up to a scalar factor, independent of the choice of the basis $S_{+}^{(a)}$. Furthermore, any product of order higher that 3 of basis vectors $S_{+}^{(a)}$ vanishes identically (e.g., $S_{+}^{(a)} \triangle S_{+}^{(b)} \triangle S_{+}^{(c)} \triangle S_{+}^{(d)}=0$ ). A volume element $W_{-}$for $S_{3}^{-}$can be similarly defined using the basis $S_{-}^{(a)}$ of $S_{3}^{-}$, which then satisfies $W_{-}=\left(W_{+}\right)^{*}$.

A 3-form $W \in \Lambda^{3}\left(S_{6}\right)$ has 20 independent components $W_{I J K}$, which correspond to a 4 -dimensional tensor $W_{i_{1} i_{2} j_{1} j_{2} k_{1} k_{2}}$ of order six, with the symmetries $W_{i_{1} i_{2} j_{1} j_{2} k_{1} k_{2}}=-W_{j_{1} j_{2} i_{1} i_{2} k_{1} k_{2}}=-W_{k_{1} k_{2} j_{1} j_{2} i_{1} i_{2}}=-W_{i_{1} i_{2} k_{1} k_{2} j_{1} j_{2}}$ and of course $W_{i_{1} i_{2} j_{1} j_{2} k_{1} k_{2}}=-W_{i_{2} i_{1} j_{1} j_{2} k_{1} k_{2}}$, etc. From this tensor, a symmetric second order 4 -dimensional tensor density of weight +1 can be defined as

$$
\mathcal{W}_{i j}:=\frac{1}{2} \epsilon^{k l m n} W_{i k l m n j}
$$

which has therefore 10 independent components. The remaining 10 components of $W$ can be mapped to a second order contravariant tensor density of weight +2 , defined by

$$
\widetilde{\mathcal{W}}^{i j}:=\frac{1}{4} \epsilon^{i k l m} W_{k l m n p q} \epsilon^{n p q j} .
$$

Following (4.80) we define the tensor density $\mathcal{W}_{i j}^{+}$associated to $W_{+}$as

$$
\mathcal{W}_{i j}^{+}:=\frac{1}{2} \epsilon^{k l m n} W_{i k l m n j}^{+}
$$

or, equivalently,

$$
\mathcal{W}_{i j}^{+}:=\frac{1}{2 \cdot 3 !} \epsilon^{k l m n} \hat{\epsilon}_{a b c} S_{i k}^{+(a)} S_{l m}^{+(b)} S_{n j}^{+(c)} .
$$

Furthermore, we saw that a change of the basis $S_{+}^{(a)}$ of $S_{3}^{+}$to a new one $S_{+}^{(a)}$ leads to a new volume $W_{+}^{\prime}$ which is necessarily proportional to $W_{+}$, i.e. $W_{+}^{\prime}=a_{3} W_{+}$for some $a_{3}$. One can therefore define a new tensor density by

$$
\hat{\mathcal{W}}_{i j}^{+}:=\left(\operatorname{det} \mathcal{W}_{k l}^{+}\right)^{-1 / 4} \mathcal{W}_{i j}^{+}
$$


Then $\hat{\mathcal{W}}_{+}$is a tensor density of weight $-1 / 2$ which is independent of the choice of the basis $S_{+}^{(a)}$ of $S_{3}^{+}$. Because of this important property, $\hat{\mathcal{W}}_{i j}^{+}$must describe an intrinsic property of the self-dual space $S_{3}^{+}$and therefore of the operator $J$. It is then tempting to identify the symmetric tensor density (4.84) with the comformally invariant part of the metric tensor $|g|^{-1 / 4} g_{i j}$, which is also a tensor density of weight $-1 / 2$, i.e.,

$$
\hat{\mathcal{W}}_{i j}^{+}=a|g|^{-1 / 4} g_{i j}
$$

with some (in general complex) factor $a$.

That this identification is consistent with our expectations can be checked as follows. Consider the linear operator $\mathcal{L}$ acting on vectors of $S_{6}(C)$, defined by

$$
(\mathcal{L} A)_{i j}:=\frac{1}{2} \mathcal{L}_{i j}{ }^{k l} A_{k l}
$$

with

$$
\mathcal{L}_{i j}{ }^{k l}:=\hat{\mathcal{W}}_{i m}^{+} \hat{\mathcal{W}}_{j n}^{+} \epsilon^{m n k l}
$$

This operator is expected to be related to the original operator $J$, because the identification (4.85) would imply

$$
\mathcal{L}_{i j}{ }^{k l}:=a^{2}|g|^{-1 / 2} g_{i m} g_{j n} \epsilon^{m n k l},
$$

which is proportional to the Hodge dual operator of the metric $g$, when applied to 2 -forms.

Using (4.84) one re-writes the definition (4.87) of the operator $\mathcal{L}$ as

$$
\mathcal{L}_{i j}{ }^{k l}=\left(\operatorname{det} \mathcal{W}_{p q}^{+}\right)^{-1 / 2} \mathcal{W}_{i m}^{+} \mathcal{W}_{j n}^{+} \epsilon^{m n k l}
$$

Furthermore. it can be proved that $\mathcal{L}$ is a complex operator. From (4.89) and the definition (4.83) one can verify by direct but rather lengthy calculations that the elements of $S_{3}^{+}$and $S_{3}^{-}$are eigenvectors of $\mathcal{L}$ with eigenvalues +1 and -1 , respectively, i.e.

$$
\mathcal{L} S^{+}=S^{+}, \quad \mathcal{L} S^{-}=-S^{-} .
$$

Since $S_{3}^{-}=\left(S_{3}^{+}\right)^{*}$, the operator $\mathcal{L}$ cannot be real. If it were $(4.90 \mathrm{a})$ would imply $\mathcal{L} S^{-}=-S^{-}$, contradicting $(4.90 \mathrm{~b})$. It is therefore clear that the real operator $J$ is just given by $J=i \mathcal{L}$, because

$$
(i \mathcal{L}) S^{+}=i S^{+}, \quad(i \mathcal{L}) S^{-}=-i S^{-}
$$


which coincides with (4.70). The property (4.91) is valid for any vector of $S_{3}^{ \pm}$and since $S_{6}(C)=S_{3}^{+} \oplus S_{3}^{-}$, it implies that the action of $i \mathcal{L}$ and $J$ on any vector of $S_{6}(C)$ is exactly the same, hence they are the same operator.

Thus, we have proved that

$$
J=i \mathcal{L} .
$$

so that, from (4.89), we have

$$
J_{i j}^{k l}=\left(-\operatorname{det} \mathcal{W}_{p q}^{+}\right)^{-1 / 2} \mathcal{W}_{i m}^{+} \mathcal{W}_{j n}^{+} \epsilon^{m n k l}
$$

With the identification (4.85) we write the operator $J$ as

$$
J_{i j}{ }^{k l}=\sqrt{-g} g_{i m} g_{j n} \epsilon^{m n k l} .
$$

From this expression, one sees that the metric $g_{i j}$ can be taken to be real, with lorentzian signature, since $J$ is a real operator. In other words, the results and (4.83) allow us to reconstruct the metric components as

$$
g_{i j} \sim \epsilon^{k l m n} \hat{\epsilon}_{a b c} S_{i k}^{+(a)} S_{l m}^{+(b)} S_{n j}^{+(c)},
$$

with $g_{i j}$ real. The conformal factor remains of course undetermined.

\section{Harnett's proof}

In ref. [23] Clifford algebra methods are used to derive the Urbantke formula. This Clifford algebra is defined in a metric-independent way, since it requires only the use of the totally antisymmetric tensor density $\epsilon$, which serves as a metric for the 6-dimensional space $S_{6}$ (of 2-forms). Therefore, the Clifford algebra is defined as that corresponding to the metric $\epsilon^{I J}, I, J, \ldots=1, \ldots, 6$, i.e., as given by six algebra elements $\gamma^{I}$ acting on a 'spin' space $S$, satisfying

$$
\left\{\gamma^{I}, \gamma^{J}\right\}=-2 \epsilon^{I J} \mathbf{1}
$$

It is a general result of the theory of Clifford algebras that for a metric space of dimension $n$ the dimension of the irreducible representation of the algebra (4.96) is of dimension $N=2^{n / 2}$ for $n$ even, and $N=2^{(n-1) / 2}$ for $n$ odd, see for instance [67], appendix B.

Thus, for instance, $N=4$ in 4-dimensional Minkowski space, corresponding to the Dirac representation of the Clifford algebra. In our case $n=6$ so that the irreducible representation is 8-dimensional. 
Natural elements constructed using the elements $\gamma^{I}$ are the totally antisymmetric objects

$$
\gamma^{I J}:=\gamma^{[I} \gamma^{J]}, \quad \gamma^{I J K}:=\gamma^{[I} \gamma^{J} \gamma^{K]}
$$

etc. Due to (4.96), other combinations of products can be written as linear combinations of the above and the identity. These totally antisymmetric objects are in correspondence to the spaces of exterior forms on the 6-dimensional linear space $\Lambda^{2}(V)$. This means that given a $p$-form $(p$ vector) $A_{[I J \ldots]}$ one can define an element of the Clifford algebra by $\gamma(A):=$ $\gamma^{I J \ldots} A_{[I J \ldots]}$.

First, an explicit representation of the Clifford algebra (4.96) is constructed. Consider, for every second order antisymmetric tensor $F_{i j}$, the linear operator $\Gamma(F) \in \operatorname{Hom}\left(V, V^{*}\right)$, i.e. an operator which, when applied to a vector $u^{i} \in V$, gives a covector $[\Gamma(F) u]_{i}:=[\Gamma(F)]_{i j} u^{j} \in V^{*}$, where

$$
[\Gamma(F)]_{i j}:=F_{i j}
$$

A second operator $\breve{\Gamma}(F) \in \operatorname{Hom}\left(V^{*}, V\right)$ is defined in an analogous way by

$$
[\breve{\Gamma}(F)]^{i j}:=\frac{1}{2} \epsilon^{i j k l} F_{k l} .
$$

Denoting each pair of antisymmetric indices $\left[i_{1} i_{2}\right]$ using the bivector index $I$, etc., we define now the quantities

$$
\Gamma_{i j}^{I}:=2 \delta_{i}^{\left[i_{1}\right.} \delta_{j}^{\left.i_{2}\right]}, \quad \breve{\Gamma}^{I i j}:=\epsilon^{i_{1} i_{2} i j},
$$

so that

$$
[\Gamma(F)]_{i j}=\Gamma_{i j}^{I} F_{I}, \quad[\breve{\Gamma}(F)]^{i j}=\breve{\Gamma}^{I i j} F_{I} .
$$

From these definitions, it is direct to verify that the following property is identically satisfied

$$
2[\Gamma(F)]_{i j}[\breve{\Gamma}(F)]^{j k}=-\delta_{i}^{k} \epsilon(F, F),
$$

where $\epsilon(F, F)$ is the inner product induced by the 'metric' $\epsilon^{I J}$ of $S_{6}$, see (4.63). Since (4.102) holds for any $F \in S_{6}$, we have

$$
2 \Gamma_{i j}^{(I} \breve{\Gamma}^{J) j k}=-\delta_{i}^{k} \epsilon^{I J} .
$$


As next step, the 8-dimensional operator $\gamma(F)$ is defined by

$$
\gamma(F):=\left(\begin{array}{cc}
0 & {[\Gamma(F)]_{i j}} \\
{[\breve{\Gamma}(F)]^{i j}} & 0
\end{array}\right),
$$

with 'components'

$$
\gamma^{I}:=\left(\begin{array}{cc}
0 & \Gamma_{i j}^{I} \\
\breve{\Gamma}^{I i j} & 0
\end{array}\right)
$$

such that, when applied on a pair $\left(\begin{array}{c}\phi_{i} \\ u^{i}\end{array}\right) \in V^{*} \oplus V$ gives a new pair $\left(\begin{array}{c}\phi_{i}^{\prime} \\ u^{i \prime}\end{array}\right) \in$ $V^{*} \oplus V$, defined by

$$
\left(\begin{array}{c}
\phi_{i}^{\prime} \\
u^{i \prime}
\end{array}\right):=\left(\begin{array}{cc}
0 & {[\Gamma(F)]_{i j}} \\
{[\breve{\Gamma}(F)]^{i j}} & 0
\end{array}\right)\left(\begin{array}{l}
\phi_{j} \\
u^{j}
\end{array}\right) .
$$

Using the identity (4.103) we find that

$$
2[\gamma(F)]^{2}=-\epsilon(F, F) \mathbf{1}_{8} .
$$

As the identity (4.107) is valid for any $F_{I}$, we obtain

$$
2 \gamma^{(I} \gamma^{J)}=-\mathbf{1}_{8} \epsilon^{I J}
$$

showing that the matrices (4.105) form an 8-dimensional (metric-free) representation of the 6-dimensional Clifford algebra (4.96). The spinors in this representation are given by the 8 -dimensional quantities on which the matrices $\gamma(F)$ act.

This explicit construction agrees with the general result that for a Clifford algebra of even dimension $n$, it is possible to find representations with the form (4.105), i.e., with block off-diagonal matrices $\gamma^{I}$. In this case the spaces on which $\Gamma_{i j}^{I}$ and $\breve{\Gamma}^{I i j}$ act are called reduced spin spaces, see [67]. In other words, the explicit representation of Harnett shows that the reduced spin spaces of the irreducible representation of the 6-dimensional Clifford algebra (4.96) correspond to the tangent and cotangent spaces of the 4-dimensional spacetime. The spinors of the representation are then direct sums of vector and covectors, i.e. $S=V^{*} \oplus V$.

Now, we compute the higher order elements $\gamma^{I J}$ and $\gamma^{I J K}$ as defined in (4.97). Direct application of their definition gives

$$
\gamma^{I J}=\left(\begin{array}{cc}
\Gamma^{I J}{ }_{i}^{j} & 0 \\
0 & \breve{\Gamma}_{j}^{I J i}
\end{array}\right),
$$


with

$$
\Gamma_{i}^{I J}{ }_{i}^{j}:=\Gamma_{i k}^{[I} \breve{\Gamma}^{J] k j}, \quad \breve{\Gamma}^{I J i}{ }_{j}:=\breve{\Gamma}^{[I|i k|} \Gamma_{k j}^{J]},
$$

and

$$
\gamma^{I J K}=\left(\begin{array}{cc}
0 & \Gamma_{i j}^{I J K} \\
\breve{\Gamma}^{I J K i j} & 0
\end{array}\right),
$$

with

$$
\Gamma^{I J K}{ }_{i j}:=\Gamma_{i k}^{[I} \breve{\Gamma}^{J|k l|} \Gamma_{l j}^{K]}, \quad \breve{\Gamma}^{I J K i j}:=\breve{\Gamma}^{[I|i k|} \Gamma_{k l}^{J} \breve{\Gamma}^{k] l j} .
$$

It follows from these definitions that $\Gamma^{I J K}{ }_{i j}$ and $\breve{\Gamma}^{I J K}{ }^{i j}$ are symmetric in the pair of spacetime indices $i j$. These objects will be directly related to the derivation of the metric tensor when the additional structure provided by a symmetric duality operator is introduced. In what follows, explicit expressions for the $\Gamma^{I J K}{ }_{i j}$ and $\breve{\Gamma}^{I J K i j}$ will be useful. From (4.111), (4.112) and $(4.100)$ we find

$$
\begin{aligned}
\Gamma^{I J K}{ }_{i j}= & \frac{1}{6}\left(\delta_{i}^{k_{1}} \delta_{j}^{j_{2}} \epsilon^{i_{1} i_{2} k_{2} j_{1}}-\delta_{i}^{k_{1}} \delta_{j}^{j_{1}} \epsilon^{i_{1} i_{2} k_{2} j_{2}}-\delta_{i}^{k_{2}} \delta_{j}^{j_{2}} \epsilon^{i_{1} i_{2} k_{1} j_{1}}\right. \\
& +\delta_{i}^{k_{2}} \delta_{j}^{j_{1}} \epsilon^{i_{1} i_{2} k_{1} j_{2}}-\delta_{i}^{i_{1}} \delta_{j}^{j_{2}} \epsilon^{k_{1} k_{2} i_{2} j_{1}}+\delta_{i}^{i_{1}} \delta_{j}^{j_{1}} \epsilon^{k_{1} k_{2} i_{2} j_{2}} \\
& \left.+\delta_{i}^{i_{2}} \delta_{j}^{j_{2}} \epsilon^{k_{1} k_{2} i_{1} j_{1}}-\delta_{i}^{i_{2}} \delta_{j}^{j_{1}} \epsilon^{k_{1} k_{2} i_{1} j_{2}}\right), \\
\breve{\Gamma}^{I J K i j}= & \frac{1}{6}\left(\epsilon^{i_{1} i_{2} i j_{1}} \epsilon^{k_{1} k_{2} j_{1} j}-\epsilon^{i_{1} i_{2} i j_{2}} \epsilon^{k_{1} k_{2} j_{2} j}-\epsilon^{i_{1} i_{2} i k_{1}} \epsilon^{j_{1} j_{2} k_{2} j}\right. \\
& +\epsilon^{i_{1} i_{2} i k_{2}} \epsilon^{j_{1} j_{2} k_{1} j}+\epsilon^{j_{1} j_{2} i k_{1}} \epsilon^{i_{1} i_{2} k_{2} j}-\epsilon^{j_{1} j_{2} i k_{2}} \epsilon^{i_{1} i_{2} k_{1} j} \\
& -\epsilon^{j_{1} j_{2} i i_{1}} \epsilon^{k_{1} k_{2} i_{2} j}+\epsilon^{j_{1} j_{2} i i_{2}} \epsilon^{k_{1} k_{2} i_{1} j}+\epsilon^{k_{1} k_{2} i i_{1}} \epsilon^{j_{1} j_{2} i_{2} j} \\
& \left.-\epsilon^{k_{1} k_{2} i i_{2}} \epsilon^{j_{1} j_{2} i_{1} j}-\epsilon^{k_{1} k_{2} i j_{1}} \epsilon^{i_{1} i_{2} j_{2} j}+\epsilon^{k_{1} k_{2} i j_{2}} \epsilon^{i_{1} i_{2} j_{1} j}\right) .
\end{aligned}
$$

With this, one can verify the identities

$$
\begin{aligned}
& \Gamma_{i j}^{I J K} \breve{\Gamma}_{I J K}^{k l}=48 \delta_{(i}^{k} \delta_{j)}^{l}, \\
& \Gamma^{I J K}{ }_{i j} \Gamma_{I J K k l}=0, \quad \breve{\Gamma}^{I J K i j} \breve{\Gamma}_{I J K}{ }^{k l}=0 .
\end{aligned}
$$

Here the 6 -dimensional indices $I, J, \ldots$ have been raised and lowered using the 'metric' $\epsilon^{I J}$. 
Given a quantity of the form $A_{I J K}=A_{[I J K]}$, i.e a 3-form of $S_{6}(20$ independent components), one can define the 'spinor' $\gamma(A)$ by $\gamma(A):=$ $\frac{1}{3 !} \gamma^{I J K} A_{I J K}$ (the same can be done for any form on the 6-dimensional space). Looking at (4.111) we see that $\gamma(A)$ defines in fact two second order symmetric (co)tensors, namely $\frac{1}{3 !} \Gamma^{I J K}{ }_{i j} A_{I J K}$ and $\frac{1}{3 !} \breve{\Gamma}^{I J K i j} A_{I J K}$. This objects are equivalent to the definitions (4.81) and (4.80) of Schönberg, respectively. This vector will provide, when applied to a volume element of a selfdual space of the dual operator $J$, the contravariant and covariant components of the metric tensor, respectively, see below.

The identities (4.115) and (4.116) can be used to prove a formula for the inverse of $\gamma(A)$. One verifies that if

$$
A_{I J K}:=\frac{1}{8}\left(\breve{\Gamma}_{I J K}^{i j} U_{i j}+\Gamma_{I J K i j} V^{i j}\right),
$$

then

$$
\gamma(A)=\left(\begin{array}{cc}
0 & U_{i j} \\
V^{i j} & 0
\end{array}\right) .
$$

With these objects, Harnett finally defines, assuming the presence of a metric $g_{i j}$, the quantity $S_{I J K}$ (a 3-form on $S_{6}$ ) defined by

$$
\gamma(S):=\left(\begin{array}{cc}
0 & i|g|^{1 / 4} g_{i j} \\
|g|^{-1 / 4} g^{i j} & 0
\end{array}\right)
$$

and the inverse formula (4.117).

The definition (4.119), and in particular the choice of the imaginary factor $i$ on the right hand side, which is necessary in the case of a lorentzian metric, is constructed such that ${ }^{* *}=-1$ and the following property is fulfilled

$$
\gamma\left({ }^{*} F\right)=\gamma(S) \gamma(F) \gamma(S),
$$

where ${ }^{*}$ is the Hodge dual operator defined by the metric, i.e.

$$
\left({ }^{*} F\right)_{i j}=\frac{\sqrt{|g|}}{2} \hat{\epsilon}_{i j k l} g^{k m} g^{l n} F_{m n} .
$$

The identity (4.120) can be directly proved computing the right hand side:

$$
\gamma(S) \gamma(F) \gamma(S)=\left(\begin{array}{cc}
0 & i|g|^{-1 / 4} g_{i j} \\
|g|^{1 / 4} g^{i j} & 0
\end{array}\right)\left(\begin{array}{cc}
0 & F_{j k} \\
\frac{1}{2} \epsilon^{j k l m} F_{l m} & 0
\end{array}\right)
$$




$$
\begin{aligned}
& \times\left(\begin{array}{cc}
0 & i|g|^{-1 / 4} g_{k n} \\
|g|^{1 / 4} g^{k n} & 0
\end{array}\right) \\
= & \left(\begin{array}{cc}
0 & i|g|^{-1 / 4} g_{i j} \\
|g|^{1 / 4} g^{i j} & 0
\end{array}\right) \\
& \times\left(\begin{array}{cc}
|g|^{1 / 4} F_{j k} g^{k n} & 0 \\
0 & \frac{i}{2}|g|^{-1 / 4} \epsilon^{j k l m} F_{l m} g_{k n}
\end{array}\right) \\
= & \left(\begin{array}{cc}
0 & -\frac{1}{2}|g|^{-1 / 2} \epsilon^{j k l m} F_{l m} g_{k n} g_{i j} \\
|g|^{1 / 2} g^{i j} F_{j k} g^{k n} & 0
\end{array}\right) \\
= & \left(\begin{array}{cc}
0 & \frac{1}{2}|g|^{1 / 2} \hat{\epsilon}_{i n j k} g^{j l} g^{k m} F_{l m} \\
|g|^{1 / 2} g^{i j} F_{j k} g^{k n} & 0
\end{array}\right) \\
= & \left(\begin{array}{cc}
0 & \left({ }^{*} F\right)_{i n} \\
\frac{1}{2} \epsilon^{i n l m}\left({ }^{*} F\right)_{l m} & 0
\end{array}\right) \\
= & \gamma\left({ }^{*} F\right) .
\end{aligned}
$$

Here we used that $g=\operatorname{det} g_{i j}<0$ for a lorentzian metric.

Finally, Harnett shows that the $S_{I J K}$ defined above is in fact a volume element of the 3-dimensional space $S_{3}^{+}(C)$ which is self-dual with respect to the Hodge dual operator of the metric. This volume element is unique up to a scalar factor and can therefore be computed using any basis of the space of self dual 2-forms. The definition (4.119) provides then explicit formulas for the (covariant and contravariant components of the) metric tensor of spacetime from a quantity that depends only on the properties of the Hodge dual.

Therefore, this approach also assumes the existence of a metric such that the dual operator corresponds to its Hodge operator. However, the proof that $S$ must be a volume element of the selfdual space is not clear. It involves the consideration of the so called 'twisted adjunct representation' and a theorem by Harvey, see [23]. This is of course, one of the essential points of this proof of the Urbantke formula.

However, once it is proven that $S$ is a volume element of the space of selfdual 2-forms (2-vectors) $S^{+}$, the Urbantke formula is found in the following way. If $S^{(a)}, a=1,2,3$ is a basis of the 3 -dimensional space $S_{3}^{+}$, then a volume element can be constructed as

$$
S_{I J K} \sim \hat{\epsilon}_{a b c} S_{I}^{(a)} S_{J}^{(b)} S_{K}^{(c)} .
$$


This quantity is unique up to an overall factor. Using this result, the associated $\gamma(S)$ consists of the metric componets $g_{i j}$ and $g^{i j}$, given by

$$
\begin{gathered}
g_{i j}=\Gamma_{i j}^{I J K} S_{I J K} \sim \Gamma^{I J K}{ }_{i j} \hat{\epsilon}_{a b c} S_{I}^{(a)} S_{J}^{(b)} S_{K}^{(c)}, \\
i g^{i j}=\breve{\Gamma}^{I J K i j} S_{I J K} \sim \breve{\Gamma}^{I J K i j} \hat{\epsilon}_{a b c} S_{I}^{(a)} S_{J}^{(b)} S_{K}^{(c)} .
\end{gathered}
$$

Finally, using (4.113) and (4.114), we find

$$
\begin{aligned}
g_{i j} & \sim \hat{\epsilon}_{a b c} \epsilon^{k l m n} S_{i k}^{(a)} S_{l m}^{(b)} S_{n j}^{(c)} \\
g^{i j} & \sim i \hat{\epsilon}_{a b c} \epsilon^{i k l m} \epsilon^{j n p q} S_{k l}^{(a)} S_{m n}^{(b)} S_{p q}^{(c)} .
\end{aligned}
$$

The derivation of Harnett seems to be then equivalent to that of Schönberg, but using the formalism of the Clifford algebra naturally defined in the 6-dimensional space $S_{6}$. In both approaches a fundamental role is played by the self-dual space defined by the duality operator, and in particular by the volume element of $S_{3}^{+}$, which is mapped in the 4-dimensional space into the conformal metric components. The formalism developed by Harnett offers, however, a more powerfull framework for the case in which one in interested in formulate the whole conformal geometry in term of the 6-dimensional quantities related to $S_{6}$.

\subsubsection{Necessary and sufficient conditions for the con- stitutive tensor}

The results discussed above can be summarized as follows. If the operator $J$ defined in (4.14) by the dimensionless part of the constitutive tensor is symmetric and defines a complex structure, i.e. (4.20) and (4.21) are satisfied, then a lorentzian metric $g$ is determined, up to a conformal factor such that

$$
\stackrel{\circ}{\chi}^{i j k l}=2 \sqrt{-g} g^{i[k} g^{l] j},
$$

with $g:=\operatorname{det} g_{i j}<0$. In other words, the conditions (4.20) and (4.21) are sufficient to be able to write $\stackrel{\circ}{\chi}$ in the form (4.128)

But it is clear, see the discussion in section 4.1 , that (4.128) is sufficient to ensure that the corresponding operator $J$ satisfies (4.20) and (4.21).

Therefore, 
The necessary and sufficient conditions to be able to write the dimensionless part $\stackrel{\circ}{\chi}$ of the constitutive tensor as

$$
\stackrel{\circ}{\chi}^{i j k l}=2 \sqrt{-g} g^{i[k} g^{l] j},
$$

with a lorentzian metric $g$ are symmetry

$$
\stackrel{o}{\chi}^{i j k l}=\stackrel{o}{\chi} k l i j
$$

and closure

$$
\frac{1}{8} \hat{\epsilon}_{\alpha \beta \gamma \delta} \stackrel{\circ}{\chi} \gamma \delta \epsilon \theta \hat{\epsilon}_{\epsilon \theta \mu \nu} \stackrel{\circ}{\chi}^{\mu \nu \lambda \rho}=-2 \delta_{\alpha}^{[\lambda} \delta_{\beta}^{\rho]} .
$$

\subsection{General solution of the Closure Relation}

In order to be able to find an explicit expression for the metric in terms of quantities describing the components of the constitutive tensor, we have to solve the closure relation (4.13), or equivalently (4.18). We will solve this equations for the general case in which the constitutive tensor is asymmetric, so that we can later investigate the effect of relaxing the symmetry condition.

Let us now make the closure relation explicit. We turn back to the constitutive $6 \times 6$ matrix (4.19). We define dimensionless $3 \times 3$ matrices $\stackrel{0}{\mathcal{A}}:=\mathcal{A} / \zeta$, etc. In terms of these dimensionless matrices (we immediately drop the small circle for convenience), the closure relation reads,

$$
\begin{aligned}
\mathcal{A}^{a c} \mathcal{B}_{c b}+\mathcal{C}^{a}{ }_{c} \mathcal{C}^{c}{ }_{b} & =-\delta_{b}^{a}, \\
\mathcal{C}^{a}{ }_{c} \mathcal{A}^{c b}+\mathcal{A}^{a c} \mathcal{D}_{c}{ }^{b} & =0 \\
\mathcal{B}_{a c} \mathcal{C}^{c}{ }_{b}+\mathcal{D}_{a}{ }^{c} \mathcal{B}_{c b} & =0 \\
\mathcal{B}_{a c} \mathcal{A}^{c b}+\mathcal{D}_{a}{ }^{c} \mathcal{D}_{c}{ }^{b} & =-\delta_{a}^{b} .
\end{aligned}
$$

In $3 \times 3$ matrix notation, we then have

$$
\begin{aligned}
\mathcal{A B}+\mathcal{C}^{2} & =-1_{3} \\
\mathcal{C} \mathcal{A}+\mathcal{A D} & =0 \\
\mathcal{B C}+\mathcal{D B} & =0 \\
\mathcal{B A}+\mathcal{D}^{2} & =-1_{3}
\end{aligned}
$$


Assume $\operatorname{det} \mathcal{B} \neq 0$. Then we can find the general non-degenerate solution as follows. Define the matrix $K_{a b}$ by

$$
K:=\mathcal{B C}, \quad \text { i.e. } \quad \mathcal{C}=\mathcal{B}^{-1} K
$$

and substitute it into (4.138), then

$$
\mathcal{D}=-K \mathcal{B}^{-1}
$$

Next, solve (4.136) with respect to $\mathcal{A}$ :

$$
\mathcal{A}=-\mathcal{B}^{-1}-\mathcal{B}^{-1} K \mathcal{B}^{-1} K \mathcal{B}^{-1}
$$

Multiply (4.142) by $\mathcal{C}$ from the left and by $\mathcal{D}$ from the right, respectively, and find with (4.140) and (4.141),

$$
\begin{aligned}
\mathcal{C A} & =-\mathcal{B}^{-1} K \mathcal{B}^{-1}-\mathcal{B}^{-1} K \mathcal{B}^{-1} K \mathcal{B}^{-1} K \mathcal{B}^{-1} \\
\mathcal{A D} & =+\mathcal{B}^{-1} K \mathcal{B}^{-1}+\mathcal{B}^{-1} K \mathcal{B}^{-1} K \mathcal{B}^{-1} K \mathcal{B}^{-1}
\end{aligned}
$$

Thus, (4.137) is automatically satisfied. Accordingly, only (4.139) has still to be checked. We compute its first and second term of its left side,

$$
\begin{aligned}
\mathcal{B A} & =-1_{3}-K \mathcal{B}^{-1} K \mathcal{B}^{-1} \\
\mathcal{D}^{2} & =K \mathcal{B}^{-1} K \mathcal{B}^{-1}
\end{aligned}
$$

and find that it is fulfilled, indeed.

Summing up, we have derived the general solution of the closure relation (4.19) in terms of two arbitrary matrices $\mathcal{B}$ and $K$ as

$$
\begin{aligned}
\mathcal{A} & =-\mathcal{B}^{-1}-\mathcal{B}^{-1} K \mathcal{B}^{-1} K \mathcal{B}^{-1} \\
\mathcal{C} & =\mathcal{B}^{-1} K \\
\mathcal{D} & =-K \mathcal{B}^{-1}
\end{aligned}
$$

or, in components,

$$
\begin{aligned}
\mathcal{A}^{a b} & =-\mathcal{B}^{a b}-\mathcal{B}^{a c} K_{c d} \mathcal{B}^{d e} K_{e f} \mathcal{B}^{f b} \\
\mathcal{B}^{a b} & =\mathcal{B}^{a b} \\
\mathcal{C}^{a}{ }^{b} & =\mathcal{B}^{a c} K_{c b} \\
\mathcal{D}_{a}{ }^{b} & =-K_{a c} \mathcal{B}^{c b} .
\end{aligned}
$$


Here $\mathcal{B}^{a b}$ are the components of the inverse $\mathcal{B}^{-1}$, i.e. $\mathcal{B}^{a b} \mathcal{B}_{b c}=\delta_{b}^{a}$. The solution thus has $2 \times 9=18$ independent components. Alternatively, one can write the solution in a more compact notation, as

$$
\begin{aligned}
\mathcal{A} & =-\left(1_{3}+\mathcal{C}^{2}\right) \mathcal{B}^{-1} \\
\mathcal{D} & =-\mathcal{B C B}^{-1}
\end{aligned}
$$

which is parametrized by the arbitrary matrices $\mathcal{B}$ and $\mathcal{C}$ with altogether 18 independent components. In components, this means

$$
\begin{aligned}
\mathcal{A}^{a b} & =-\left(\delta_{d}^{a}+\mathcal{D}_{c}{ }^{a} \mathcal{D}_{d}{ }^{c}\right) \mathcal{B}^{d b} \\
\mathcal{B}_{a b} & =\mathcal{B}_{a b} \\
\mathcal{C}^{b}{ }_{a} & =-\mathcal{B}_{a c} \mathcal{D}_{d}{ }^{c} \mathcal{B}^{d b} \\
\mathcal{D}_{b}{ }^{a} & =\mathcal{D}_{b}{ }^{a}
\end{aligned}
$$

\subsubsection{Explicit derivation of the metric components from the constitutive tensor}

Here, the Schönberg-Urbantke formula will be used to find an explicit expression for the conformal metric in terms of quantities parametrizing a constitutive tensor satisfying the assumptions of symmetry and closure. From the symmetry condition one finds that the constitutive tensor has the form (2.73), but with

$$
\mathcal{A}^{a b}=\mathcal{A}^{b a}, \quad \mathcal{B}_{a b}=\mathcal{B}_{b a}, \quad \mathcal{D}_{b}{ }^{a}=\mathcal{C}^{a}{ }_{b}
$$

so that ${ }^{(2)} \chi=0$, see $(2.75)$. These symmetry conditions restrict the general solution of the closure relation found in the last section. From (4.152) and (4.153) we see that (4.161c) implies $-K_{b c} \mathcal{B}^{c a}=\mathcal{B}^{a c} K_{c b}$. Multiplying this equation by $\mathcal{B}_{a d}$ and using (4.161c) we obtain $-K_{b d}=K_{d b}$ which tells us that the matrix $K$ must be antisymmetric. Then (4.150) implies that $\mathcal{A}$ is automatically symmetric, so no further conditions on $K$ arise from (4.161a). In summary, a symmetric constitutive tensor satisfying the closure relation is given by (4.150)-(4.153) with

$$
\mathcal{B}_{a b}=\mathcal{B}_{b a}, \quad K_{a b}=-K_{b a}
$$


As we saw in section 4.3.2, to construct the metric using the SchönbergUrbantke formula, we need a basis of the 3-dimensional space $S_{3}^{+}$of self-dual 2 -forms of the duality operator $J$.

To that end, we decompose the basis $b^{I}$ of $S_{6}$ into two 3-dimensional column vectors, according to

$$
b^{I}=\left(\begin{array}{c}
\beta^{a} \\
\gamma_{b}
\end{array}\right), \quad a, b, \ldots=1,2,3 .
$$

Then we can find their self-dual parts,

$$
b_{+}^{I}=\frac{1}{2}\left(b^{I}-i J b^{I}\right),
$$

and decompose them similarly as in (4.163), into

$$
b_{+}^{I}=\left(\begin{array}{c}
\beta_{+}^{a} \\
\gamma_{b}^{+}
\end{array}\right) \text {. }
$$

Assuming, as we did before to find a solution of the closure relation, that the matrix $\mathcal{B}$ is nonsingular, we can show that one can take $\gamma^{+}$as a basis of the self-dual space $S_{3}^{+}$, since the remaining self-dual 2 -forms $\beta_{+}$can be written as linear combinations of the former. This is expected since $S_{3}$ is 3-dimensional. Explicitly, we have

$$
\beta_{+}^{a}=\left(i \delta_{b}^{a}+\mathcal{B}^{a c} K_{c b}\right) \mathcal{B}^{b d} \gamma_{d}^{+} .
$$

This allows us to choose the 2-forms $\gamma_{a}^{+}$or, equivalently, the triplet

$$
S_{+}^{a}:=-\mathcal{B}^{a b} \gamma_{b}^{+}
$$

as basis of $S_{3}^{+}$.

The information of the constitutive tensor $\chi$ is now encoded into the triplet of 2-forms $S_{+}^{(a)}$.

The $\left(S_{+}^{a}\right)_{i j}$ are the components of the 2-form triplet $S_{+}^{a}=\left(S_{+}^{a}\right)_{i j} d x^{i} \wedge$ $d x^{i} / 2$. If we substitute the self-dual 2 -forms $S_{+}^{a}$ into (4.95), we can display the metric explicitly in terms of the constitutive coefficients:

$$
g_{i j}=\phi\left(\begin{array}{c|c}
\operatorname{det} \mathcal{B} & -k_{a} \\
\hline-k_{b} & -\mathcal{B}_{a b}+(\operatorname{det} \mathcal{B})^{-1} k_{a} k_{b}
\end{array}\right) .
$$

Here $k^{a}:=\epsilon^{a b c} K_{b} c / 2, k_{a}:=\mathcal{B}_{a b} k^{b}$ and $\phi$ is an arbitrary factor. The determinant of this metric is found to be $g=-\phi^{4}(\operatorname{det} \mathcal{B})^{2}$ so that we verify that the metric in (4.168) has lorentzian signature. 


\subsubsection{Properties of the metric}

The inverse of (4.168) can be found to be

$$
g^{i j}=\frac{1}{\phi \operatorname{det} \mathcal{B}}\left(\begin{array}{c|c}
1-(\operatorname{det} \mathcal{B})^{-1} k_{c} k^{c} & -k^{b} \\
\hline-k^{a} & -(\operatorname{det} \mathcal{B}) \mathcal{B}^{a b}
\end{array}\right) .
$$

With the help of (4.168) and (4.169), we can compute the Hodge duality operator $^{*}$ attached to this metric. In terms of the components of the 2 -form $F$, we have

$$
{ }^{*} F_{i j}:=\frac{\sqrt{-g}}{2} \hat{\epsilon}_{i j k l} g^{k m} g^{l n} F_{m n}
$$

This equation can be rewritten, in analogy to (2.45), by using the constitutive tensor $\chi_{\{g\}}$ defined in (4.3), so that

$$
{ }^{*} F_{i j}=\frac{1}{4} \hat{\epsilon}_{i j k l} \chi_{\{g\}}^{k l m n} F_{m n},
$$

In order to compare $\chi_{\{g\}}$ with the original constitutive tensor $\chi$, we compute the corresponding 3 -dimensional constitutive matrices of $\chi_{\{g\}}$ according to $(2.64)$ and (2.64).

Then straightforward calculations yield

$$
\begin{aligned}
\mathcal{A}_{\{g\}}^{a b} & =\sqrt{-g}\left(g^{00} g^{a b}-g^{0 a} g^{0 b}\right)=\mathcal{A}^{a b}, \\
\left(\mathcal{B}_{\{g\}}\right)_{a b} & =\frac{1}{4} \sqrt{-g}\left(g^{c e} g^{d f}-g^{d e} g^{e f}\right) \hat{\epsilon}_{a c d} \hat{\epsilon}_{e f b}=\mathcal{B}_{a b}, \\
\left(\mathcal{C}_{\{g\}}\right)_{b}^{a} & =\frac{1}{2} \sqrt{-g}\left(g^{0 c} g^{a d}-g^{a c} g^{0 d}\right) \hat{\epsilon}_{b c d}=\mathcal{B}^{a d} K_{d b}=C_{b}^{a} .
\end{aligned}
$$

Thus, $\chi_{\{g\}}=\chi$, i.e., the metric extracted allows us to write the original duality operator $J$ as Hodge duality operator, $J={ }^{*}$, when applied on 2forms.

\subsubsection{Alternative derivation}

Here we provide an alternative derivation of the conformal metric, this time based on a direct computation of the Fresnel equation for the symmetric solution of the closure relation given by $(4.150),(4.153)$ and $(4.162)$. Using this equation and our general expressions (3.34)-(3.37) for the Fresnel 
equation in terms of the 3-dimensional constitutive matrices, we find after a straightforward calculation that

$$
\begin{aligned}
M & =-\frac{1}{\operatorname{det} \mathcal{B}}\left(1-\frac{k_{a} k^{a}}{\operatorname{det} \mathcal{B}}\right)^{2} \\
M^{a} & =\frac{1}{\operatorname{det} \mathcal{B}} 4 k^{a}\left(1-\frac{k_{b} k^{b}}{\operatorname{det} \mathcal{B}}\right) \\
M^{a b} & =-\frac{1}{\operatorname{det} \mathcal{B}} 4 k^{a} k^{b}+2 \mathcal{B}^{a b}\left(1-\frac{k_{c} k^{c}}{\operatorname{det} \mathcal{B}}\right), \\
M^{a b c} & =-4 \mathcal{B}^{b(a} k^{c)} \\
M^{(a b c d)} & =-(\operatorname{det} \mathcal{B}) \mathcal{B}^{(a b} \mathcal{B}^{c d)} .
\end{aligned}
$$

Substituting all this into the general Fresnel equation (3.30), we find

$$
\begin{array}{r}
\mathcal{W}=-\frac{\theta^{2}}{\operatorname{det} \mathcal{B}}\left[q_{0}^{2}\left(1-\frac{k_{a} k^{a}}{\operatorname{det} \mathcal{B}}\right)-2 q_{0}\left(q_{a} k^{a}\right)\right. \\
\left.-(\operatorname{det} \mathcal{B})\left(q_{a} q_{b} \mathcal{B}^{a b}\right)\right]^{2}
\end{array}
$$

Therefore we find that the Fresnel equation, $\mathcal{W}=0$, can be written as

$$
\left(g^{i j} q_{i} q_{j}\right)^{2}=0
$$

where $g^{i j}$ is the (inverse) 4-dimensional conformal metric which arises from the duality operator and the closure relation. Direct comparison with (4.180) shows that

$$
\begin{aligned}
g^{00} & =\psi\left(1-\frac{k_{a} k^{a}}{\operatorname{det} \mathcal{B}}\right) \\
g^{0 a} & =-\psi k^{a} \\
g^{a b} & =-\psi(\operatorname{det} \mathcal{B}) \mathcal{B}^{a b}
\end{aligned}
$$

Here $\psi$ in the undertermined conformal factor.

Thus we indeed recover the null cone structure for the propagation of electromagnetic waves from our general analysis. The quartic surface degenerates to the null cone for the conformal metric $g$. 


\subsection{Relaxing the symmetry condition}

Having verified and explicitly constructed the metric tensor from the constitutive tensor in the case in which it is symmetric and satisfies the closure relation, we want to study what consequences on the emergence of a conformal structure a nonvanishing skewon piece in the constitutive tensor could have. In particular, can the spacetime metric still be constructed? We have seen that the skewon piece does influence the lightcone structure, and we also saw that an asymmetric constitutive tensor satisfying closure can accommodate 18 independent functions. Could this case for instance correspond to the emergence of two lightcones, each with its 9 independent components?

Unfortunately, a computation of the Fresnel tensor and the Fresnel equation using the general asymmetric solution (4.150) of the closure relation does not help much to recognize a possible double light cone structure. The Fresnel equation is still a quartic equation, in general.

In what follows, we will study a particular case to try to get an idea of the qualitative properties that an additional skewon piece can induce. We will consider the case in which $K=0$, which implies $\mathcal{D}=0, \mathcal{C}=0^{2}$ and $\mathcal{A}=-\mathcal{B}^{-1}$. Furthermore, we decompose the arbitrary matrix $\mathcal{B}$ into its symmetric and antisymmetric parts,

$$
\mathcal{B}_{a b}=b_{a b}+\hat{\epsilon}_{a b c} n^{c}, \quad \text { with } \quad b_{a b}:=\mathcal{B}_{(a b)}, \quad n^{c}:=\epsilon^{c a b} B_{[a b]} .
$$

Note that $b_{a b}$ contributes to ${ }^{(1)} \chi$ and $n^{c}$ to ${ }^{(2)} \chi$. Now we can lower the index of $n^{a}$ by means of $b_{a b}$, namely, $n_{a}:=b_{b c} n^{c}$ and $n^{2}:=n^{c} n_{c}=b_{a b} n^{a} n^{b}$, and, provided det $b \neq 0$, we can raise an index by $b^{a b}$ which denotes the inverse of $b_{a b}$. We find $\operatorname{det} \mathcal{B}=\operatorname{det} b+n^{2}$, and the inverse of (4.185a) reads

$$
\mathcal{B}^{a b}=\frac{1}{\operatorname{det} b+n^{2}}\left(\bar{b}^{a b}+n^{a} n^{b}-\epsilon^{a b c} n_{c}\right) .
$$

Here the symmetric matrix $\bar{b}^{a b}$ is the matrix of the minors of $b_{a b}$. If $\operatorname{det} b \neq 0$, then $\bar{b}^{a b}=b^{a b} \operatorname{det} b$.

Then, by substituting (4.185) into (3.30), we obtain:

$$
\mathcal{W}=a\left[q_{0}^{4}-2 q_{0}^{2}\left(q^{2} \operatorname{det} b-(q n)^{2}\right)+\left(q^{2} \operatorname{det} b+(q n)^{2}\right)^{2}\right]
$$

Here we used the abbreviation $(q n):=q_{a} n^{a}$ and $a:=-\frac{q_{0}^{2}}{\left(\operatorname{det} b+n^{2}\right)}$.

\footnotetext{
${ }^{2}$ i.e., a medium with no 'magneto-electrical' properties.
} 
In general, this expression is neither a square of a quadratic polynomial nor a product of two quadratic polynomials. In other words, neither a lightcone nor a birefringence (double lightcone) structure arises generically. In order to study the reduction conditions, let us assume that the Fresnel equation is a product of two quadratic equations for $q_{i}$, i.e., the spacetime 'medium' is birefringent. Accordingly, for (4.187) we make the general ansatz

$$
\begin{aligned}
\mathcal{W} & =-\frac{q_{0}^{2}}{\left(\operatorname{det} b+n^{2}\right)}\left(q_{0}^{2}-\alpha\right)\left(q_{0}^{2}-\beta\right) \\
& =-\frac{q_{0}^{2}}{\left(\operatorname{det} b+n^{2}\right)}\left[q_{0}^{4}-(\alpha+\beta) q_{0}^{2}+\alpha \beta\right],
\end{aligned}
$$

with some polynomials $\alpha$ and $\beta$ of order 2 in $q_{a}$. This implies the relations

$$
\alpha+\beta=2\left(\bar{q}^{2}-(q n)^{2}\right), \quad \alpha \beta=\left(\bar{q}^{2}+(q n)^{2}\right)^{2},
$$

with $\bar{q}^{2}:=q_{a} q_{b} \bar{b}^{a b}$. Since $\alpha$ and $\beta$ enter symmetrically in (4.190), the solutions of this nonlinear system can be given in the form

$$
\alpha=-\left[(q n)+\sqrt{-\bar{q}^{2}}\right]^{2}, \quad \beta=-\left[(q n)-\sqrt{-\bar{q}^{2}}\right]^{2} .
$$

Thus, the question of the reducibility of the Fresnel equation translates into the algebraic problem of whether the square root $\sqrt{-\bar{q}^{2}}$ is a real linear polynomial in $q_{a}$. There are three cases, depending on the rank of the $3 \times 3$ matrix $b_{a b}$.

(i) When $b_{a b}$ has rank 3 , in other words, when $\operatorname{det} b \neq 0$, then we can write $\bar{q}^{2}=q_{a} q_{b} b^{a b} \operatorname{det} b$, and the general conclusion is that no factorization into light cones is possible (the roots $\alpha$ are complex), unless $n^{a}=0$. This latter condition implies that the constitutive tensor is symmetric, and the previous results are recovered.

(ii) When $b_{a b}$ has rank 2 , i.e., $\operatorname{det} b=0$, but at least one of the minors is nontrivial. Then, without loss of generality, we can assume the following structure of the matrix $b$ :

$$
b_{a b}=\left(\begin{array}{ccc}
b_{11} & b_{12} & 0 \\
b_{12} & b_{22} & 0 \\
0 & 0 & 0
\end{array}\right) .
$$

Its only non-vanishing minor is

$$
\bar{b}^{33}=\left|\begin{array}{ll}
b_{11} & b_{12} \\
b_{21} & b_{22}
\end{array}\right|=b_{11} b_{22}-b_{12}^{2} \neq 0 .
$$


Note that (4.192) is the most general form of a rank 2 matrix $b_{a b}$, up to a renaming of the coordinates. In order to avoid complex solutions, we have to assume that the minor $\bar{b}^{33}=-\mu^{2}<0$, so that $\sqrt{-\bar{q}^{2}}=\mu q_{3}$. Then (4.191) leads to

$$
\begin{aligned}
& \alpha=-\left[q_{1} n^{1}+q_{2} n^{2}+q_{3}\left(n^{3}+\mu\right)\right]^{2}, \\
& \beta=-\left[q_{1} n^{1}+q_{2} n^{2}+q_{3}\left(n^{3}-\mu\right)\right]^{2} .
\end{aligned}
$$

The interpretation is clear: we have birefringence, i.e., two light cones. In this case, $\mathcal{W}$ is found to be

$$
\begin{aligned}
\mathcal{W}= & -\frac{q_{0}^{2}}{b_{11}\left(n^{1}\right)^{2}+2 b_{12} n^{1} n^{2}+b_{22}\left(n^{2}\right)^{2}} \\
& \times\left[q_{0}^{2}+\left(q_{1} n^{1}+q_{2} n^{2}+q_{3}\left(n^{3}+\mu\right)\right)^{2}\right] \\
& \left.\times\left[q_{0}^{2}+\left(q_{1} n^{1}+q_{2} n^{2}+q_{3}\left(n^{3}-\mu\right)\right)^{2}\right)\right] .
\end{aligned}
$$

Then we can read off, up to conformal factors, the components of the two corresponding 'metric' tensors defining the light cones:

$$
\begin{aligned}
& g_{1}^{i j}=\left(\begin{array}{cccc}
1 & 0 & 0 & 0 \\
0 & \left(n^{1}\right)^{2} & n^{1} n^{2} & n^{1}\left(n^{3}+\mu\right) \\
0 & n^{1} n^{2} & \left(n^{2}\right)^{2} & n^{2} n^{3} \\
0 & n^{1}\left(n^{3}+\mu\right) & n^{2} n^{3} & \left(n^{3}+\mu\right)^{2}
\end{array}\right), \\
& g_{2}^{i j}=\left(\begin{array}{cccc}
1 & 0 & 0 & 0 \\
0 & \left(n^{1}\right)^{2} & n^{1} n^{2} & n^{1}\left(n^{3}-\mu\right) \\
0 & n^{1} n^{2} & \left(n^{2}\right)^{2} & n^{2} n^{3} \\
0 & n^{1}\left(n^{3}-\mu\right) & n^{2} n^{3} & \left(n^{3}-\mu\right)^{2}
\end{array}\right) .
\end{aligned}
$$

We can verify that $\operatorname{det}\left(g_{1}^{i j}\right)=\operatorname{det}\left(g_{2}^{i j}\right)=\left(n^{1}\right)^{2}\left(n^{2}\right)^{2} \bar{b}^{33}=-\left(n^{1}\right)^{2}\left(n^{2}\right)^{2} \mu^{2}<0$, so that both metrics have the correct Lorentzian signature.

(iii) When the $3 \times 3$ matrix $b_{a b}$ has rank 1 . In this case all the minors are zero, i.e., $\bar{q}^{a b}=0$, which corresponds to the case 2 for $\mu=0$. We then see that the Fresnel equation reduces to a single light cone, but the resulting metric is degenerated, since $\operatorname{det}\left(g^{i j}\right)=0$.

Thus, we have demonstrated that some of the asymmetric solutions of the closure relation can yield birefringence. 
We saw that the conditions of closure and symmetry of $\chi$ are sufficient for the existence of a well-defined light cone structure. If any of these conditions is violated, the light cone structure seems to be lost. The necessary conditions have still to be found. 


\section{Chapter 5}

\section{Conclusions and Prospects}

In this work, we have developed a general framework for describing classical electrodynamics in a general 4-dimensional medium. We have paid special attention to identify the structures which are metric-independent, and which can therefore be applied to a great variety of particular cases, from classical optics in material media, to generalized models describing the electromagnetic properties of spacetime.

One of the central results of this work is summarized in the Fresnel equation (3.29) and in particular in the Fresnel tensor (3.28). This equation determines the local properties of the propagation of waves, in particular it describes the geometry of the wave covectors. It is a remarkable result that this important equation could be derived for any linear medium. Furthermore, the result can also be used to describe the effective properties of electromagnetic perturbations in nonlinear media. Its structure, i.e., its dependence on the constitutive tensor of the corresponding medium is highly nontrivial. Cubic structures are not very common in physics. To the best of my knowledge, no previous derivation of the Fresnel equation has been given, which is as general as that in section 3.2.

It is also important to emphasize that the Fresnel equation and the whole formalism is generally covariant. This means in particular that no artificial or particular coordinate choices are necessary and that only quantities describing intrinsic properties, in our case the constitutive tensor, of the physical system under consideration enter in the formalism. Of course, inside a given medium, for example, one can choose particular coordinates which are useful for concrete calculations. This is usually the case if the medium possess some symmetry, as defined in section 2.7.1. Then an adapted coordinate system 
can be used which exploits this symmetry, so that calculations become simpler. This is, however, only a convenient choice, determined by the properties of the system (the constitutive tensor), but not a necessary a priori ingredient. In a material medium which is not isotropic, for instance, there is no a priori reason to use cartesian coordinates, the later are rather coordinates adapted to an isotropic medium, as for instance the minkowskian vacuum. Therefore, it is highly satisfactory to be able to describe the electromagnetic properties of a material medium in a generally covariant way.

We also studied the properties of the three different irreducible pieces which a general constitutive tensor can contain. We have proved that the first, symmetric, piece ${ }^{(1)} \chi$ is essential for the medium to admit well behaved wave properties. This piece is therefore the most important principal object determining the geometry of wave covectors, and the lightcone structure under some particular conditions. The second piece ${ }^{(2)} \chi$ was shown to describe dissipative properties of the medium. A nonvanishing ${ }^{(2)} \chi$ piece can lead to dissipation of electromagnetic energy, even is the material is time independent. Furthermore, the skewon piece does influence the wave propagation and therefore the lightcone structure. We have shown, studying some particular cases, that the skewon field can even give rise to a double lightcone structure. The third possible irreducible piece ${ }^{(3)} \chi$ of a constitutive tensor, the abelian axion, is more elusive. It does not contribute to the energymomentum density of the electromagnetic field, and does not affect the local properties of the electromagnetic waves and therefore the lightcone structure. It is possible, however, that it could influence the properties of wave propagation over finite (long) distances. Examples of all the three irreducible pieces of a constitutive can be found in the literature. The skewon field has been discussed so far only for material media violating $C P$ symmetry, but so far not as a model for the classical electromagnetic properties of spacetime. If one follows the common assumption that a fundamental physical system should be describable from an action principle, then the detection of a skewon piece could be interpreted as indicating an underlying substructure of the system under study. Of course, we do not claim here that the skewon field ist actually nonvanishing in spacetime. We however believe that it is a possible piece which can be used to quantify well defined, in general dissipative, properties of spacetime. Additionally, the skewon piece can be used to model possible effects violating local Lorentz invariance. Within the framework of GR, where a metric is present, one can show that the skewon piece necessarily violates local Lorentz invariance, see appendix D. 
Furthermore, we have studied the conditions under which a lightcone structure is induced. We have seen that if the constitutive tensor is symmetric and satisfies a closure relation, a lightcone is in fact induced. We also saw that the necessary and sufficient conditions for a constitutive tensor to be written as proportional to the Hodge dual operator of some metric are symmetry and closure. This one-to-one relationship is valid when we formulate it in terms of the constitutive tensor. However, it is still an open question whether symmetry and closure are necessary conditions for defining a lightcone, in the sense that the quartic Fresnel equation reduces to a quadratic equation for the wave covectors, as discussed at the beginning of chapter 4 . We proved that symmetry and closure are sufficient conditions for such reduction. Strictly speaking closure of the whole constitutive tensor is not a necessary condition since an additional axion piece does not disturb the Fresnel equation but violates closure. However, it may be that closure of only the principal irreducible piece ${ }^{(1)} \chi$ and symmetry, i.e. ${ }^{(2)} \chi=0$, could be the necessary conditions. A proof of this conjecture is, however, still missing. Non-trivial examples supporting this conjecture can be found in [64].

Other interesting open issues which could be investigated are: We have seen that the constitutive tensor defines a certain generalization of the conformal properties of spacetime. Does it also defines a (generalized) affine structure?. To answer this question, it would be interesting to study some additional properties of the propagation of waves, for instace, how does the polarization vector propagates along a light ray?. It should be possible to find an answer to this question within our general formalism and it is expected to involve some affine properties (under which parallel displacement is the polarization vector constant along the light ray?).

\subsection{A possible dynamical theory for the con- stitutive tensor?}

After developing the framework of linear pre-metric electrodynamics, and having shown that, under favorable circumstances, the conformal metric can be constructed from the constitutive tensor of the medium, one wonders if it could be possible to develop a dynamical theory of spacetime in which the constitutive tensor, and not the metric, is considered to be the fundamental dynamical variable. 
It is also clear from the previous work that a possible dynamical theory of spacetime as a linear electromagnetic medium can easily accommodate phenomena beyond GR (e.g., birefringence), maintaining the possibility to recover GR and its riemannian geometry in some limit.

Incidentally, it is interesting to notice in this context that GR was developed based on a description of spacetime in terms of riemannian geometry. The later can be considered as a generally covariant theory of 4-dimensional curved 'surfaces' (manifolds), in which the metric tensor is the fundamental variable describing the intrinsic properties of the 'surface'. One then wonders if it could be possible to describe spacetime based on a generally covariant theory of 4-dimensional inhomogeneous 'material'.

Here I speculate about this possibility and present a toy model to be studied in the future.

Of course, the first problem which comes to mind is how to recover riemannian geometry in a dynamical theory for the constitutive tensor.

Consider the Lagrange density

$$
\mathcal{L}(\chi, \Gamma, F)=G^{-1 / 4} \mathcal{G}^{i j k l} R_{i j} R_{k l}-\frac{1}{4} \chi^{i j k l} F_{i j} F_{k l} .
$$

Here $R_{i j}:=\operatorname{Ric}_{i j}(\Gamma)$ is the Ricci tensor of a connection $\Gamma_{i j}{ }^{k}$ (consider first a symmetric one) taken as a second independent dynamical field, $\mathcal{G}$ is the Fresnel tensor defined in (3.28) and $G$ is the 'generalized determinant' of $\mathcal{G}$, defined by

$$
G:=\frac{1}{4 !} \hat{\epsilon}_{i_{1} i_{2} i_{3} i_{4}} \hat{\epsilon}_{j_{1} j_{2} j_{3} j_{4}} \hat{\epsilon}_{k_{1} k_{2} k_{3} k_{4}} \hat{\epsilon}_{l_{1} l_{2} l_{3} l_{4}} \mathcal{G}^{i_{1} j_{1} k_{1} l_{1}} \mathcal{G}^{i_{2} j_{2} k_{2} l_{2}} \mathcal{G}^{i_{3} j_{3} k_{3} l_{3}} \mathcal{G}^{i_{4} j_{4} k_{4} l_{4}}
$$

Notice that $G$ is a scalar. Therefore, the Lagrange density above, which is a scalar density of weight +1 as it should be, depends only on the constitutive tensor, the connection and the electromagnetic field strength.

This choice of a Lagrangian was motivated by the work of Tapia [89]. In [89] it was shown that, in vacuum $(F=0)$, the tensor $\mathcal{G}$ must reduce such that $\mathcal{G}^{i j k l} \propto g^{(i j} g^{k l)}$, recovering the (conformal) metric. Furthermore, the equations obtained by variation with respect to the connection are then equivalent to some conformal Einstein equations with cosmological constant. However, in [89] the 'fourth order metric' $\mathcal{G}$ is assumed to be fundamental. In our case, it has substructure in terms of the constitutive tensor. But, since its dependence is algebraic in $\chi$ and $\mathcal{G}$ also appears algebraically in (5.1), it is to be expected that in our case the reduction also takes place, at least 
in vacuum. Furthermore, in our context is it clear how the coupling to the electromagnetic field has to be included (last term in (5.1)). In the model [89] this was not possible since $\mathcal{G}$ was assumed to be fundamental.

Theses possibilities, considered as a toy model, look interesting, but the field equations must be computed in detail. 


\section{Appendix A}

\section{The situation so far}

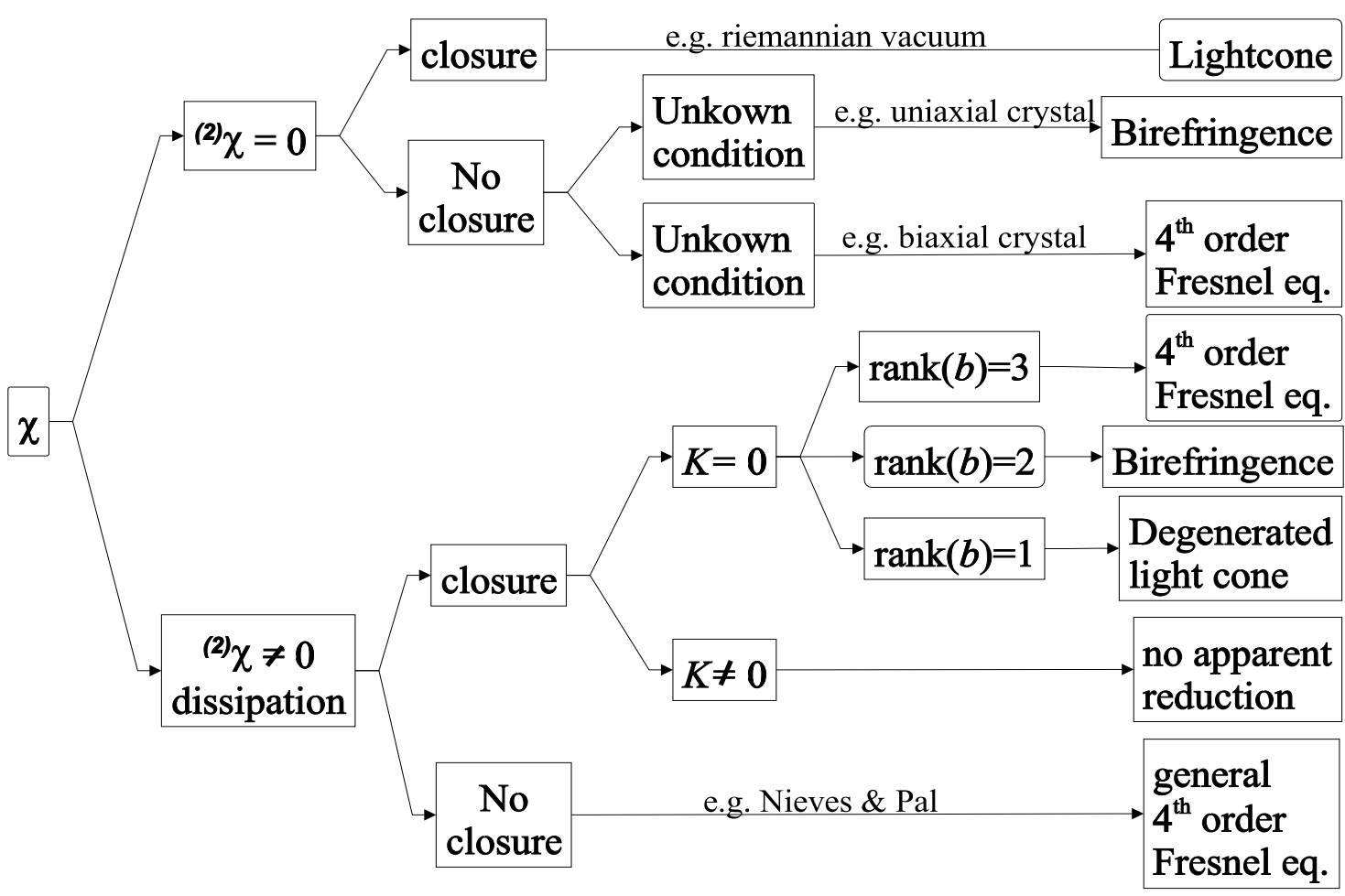

Figure A.1: Different subcases summarized. 


\section{Appendix B}

\section{Electrodynamics in a material medium}

In a material medium, treated as a continuum, one can also describe the properties of electromagnetic fields by means of macroscopic Maxwell's equations. A material medium, can be defined in general terms as a region which is neutral at macroscopic scales, but with a microscopic charge substructure. This substructure corresponds to the individual electrons, ions, etc. which form the medium. The particular distribution and dynamics of the charges forming the material are however in general unknown or difficult to model. Therefore, it is useful to describe the electrodynamics of materials only in term of the so called 'external' charges, which are those that can be, in principle, manipulated in experiments.

The procedure to define macroscopic Maxwell equations for the material medium starting from the microscopic ones consist then in the separation of the total charge in the sum of the two contributions originating 'from the inside' (bound or polarizational charge) and 'from the outside' (free or external charge):

$$
J=J^{\mathrm{mat}}+J^{\mathrm{ext}}
$$

Bound charges and bound currents are inherent characteristics of matter determined by the medium itself. In contrast, external charges and external currents appear in general outside and inside matter and can be prepared for a specific purpose.

The external charge is assumed to be conserved, and consequently the 
bound charge is conserved, too:

$$
d J^{\mathrm{mat}}=0, \quad d J^{\mathrm{ext}}=0 .
$$

As in the case of vacuum, see Refs. [63, 28, 31], this allows us to introduce the corresponding excitation $H^{\text {mat }}$ as a 'potential' for the bound current:

$$
d H^{\mathrm{mat}}=J^{\mathrm{mat}} .
$$

In a $(1+3)$ decomposition, the 6 components of $H^{\text {mat }}$ are identified with the polarization $P$ and magnetization $M$.

Defining now the external excitation $H^{\text {ext }}$ as

$$
H^{\mathrm{ext}}:=H-H^{\mathrm{mat}},
$$

we find the inhomogeneous Maxwell equation

$$
d H^{\mathrm{ext}}=J^{\mathrm{ext}} .
$$

Here, only external quantities are involved.

It remains to specify the constitutive law. In addition to the spacetime relation, $H=H(F)$, the knowledge of the internal structure of a medium yields the macroscopic excitation $H^{\text {mat }}$ (i.e., the polarization and magnetization) as a function of the electromagnetic field strength $F$ (and possibly of some thermodynamical variables describing the material continuum). Then the constitutive law of the material is given by

$$
H^{\text {ext }}=H^{\text {ext }}(F)=H(F)-H^{\text {mat }}(F) .
$$




\section{Appendix C}

\section{Some exterior and tensor calculus}

\section{C.1 Tensor and tensor densities}

A (twisted) tensor density $T$ of weight $w$ and type $\left[\begin{array}{l}p \\ q\end{array}\right]$ can be defined as an object with components $T^{i_{1} \cdots i_{p}}{ }_{j_{1} \cdots j_{q}}$ in a given coordinate system $x^{i}$ and which under a coordinate transformation $x^{i} \rightarrow x^{i^{\prime}}$ transforms as

$$
\begin{aligned}
T_{{ }_{1} \cdots j_{q}^{\prime}}^{i_{1}^{\prime} \cdots i_{p}^{\prime}}= & {\left[\operatorname{sgn}\left(\frac{\partial x^{k}}{\partial x^{k^{\prime}}}\right)\right]^{s}\left|\frac{\partial x^{k}}{\partial x^{k^{\prime}}}\right|^{w} \frac{\partial x^{i_{1}^{\prime}}}{\partial x^{i_{1}}} \cdots \frac{\partial x^{i_{p}^{\prime}}}{\partial x^{i_{p}}} } \\
& \times \frac{\partial x^{j_{1}}}{\partial x^{j_{1}^{\prime}}} \cdots \frac{\partial x^{j_{q}}}{\partial x^{j_{q}^{\prime}}} T_{{ }^{i_{1} \cdots j_{q}}}^{i_{1} \cdots i_{p}}
\end{aligned}
$$

If $s=1$, we say that $T$ is a twisted tensor density, if $s=0$ then $T$ is a normal or untwisted tensor density. If $w=0$ and $s=0$ we refer to $T$ simply as a tensor of type $\left[\begin{array}{l}p \\ q\end{array}\right]$.

The Levi-Civita symbol $\epsilon^{i j k l}$ is defined by the properties

$$
\epsilon^{i j k l}=\epsilon^{[i j k l]}, \quad \epsilon^{0123}:=1,
$$

in any coordinate system. It can be shown that it is a twisted tensor density of weight +1 . Analogously, the Levi-Civita twisted tensor density of weight -1 is defined by

$$
\hat{\epsilon}_{i j k l}=\hat{\epsilon}_{[i j k l]}, \quad \hat{\epsilon}_{0123}:=1 \text {. }
$$


Notice that these tensor densities are metric-independent. They can be defined in any 4-dimensional manifold. In particular, $\hat{\epsilon}_{i j k l}$ is not the tensor density obtained by lowering the indices of $\epsilon^{i j k l}$ by means of a metric. This later object, usually denoted with the same symbol $\epsilon$ is givem by $\epsilon_{i j k l}:=g_{i m} g_{j n} g_{k p} g_{l q} \epsilon^{m n p q}$ and is a tensor density of weight +1 . It is related to our $\hat{\epsilon}_{i j k l}$ by $\epsilon_{i j k l}=\operatorname{det}\left(g_{m n}\right) \hat{\epsilon}_{i j k l}$. Therefore, to avoid confusion, we denote the metric-independent Levi-Civita tensor density of weight -1 with an additional hat, i.e. $\hat{\epsilon}$.

\section{C.2 Lie derivative}

The Lie derivative of a tensor density of weight $w \mathcal{T}_{i_{1} \ldots i_{p}}{ }^{j_{1} \ldots j_{q}}$ along a vector field $\xi$ is defined as

$$
\begin{aligned}
\mathcal{L}_{\xi} \mathcal{T}_{i_{1} \ldots i_{p}}^{j_{1} \ldots j_{q}}:= & \xi^{k} \partial_{k} \mathcal{T}_{i_{1} \ldots i_{p} \ldots j_{q}}{ }^{j_{1}}+w\left(\partial_{k} \xi^{k}\right) \mathcal{T}_{i_{1} \ldots i_{p} \ldots j_{q}}{ }^{j_{1} \ldots j_{q}} \\
& +\left(\partial_{i_{1}} \xi^{k}\right) \mathcal{T}_{k i_{2} \ldots i_{p}}{ }^{j_{1} \ldots j_{q}}+\left(\partial_{i_{2}} \xi^{k}\right) \mathcal{T}_{i_{1} \ldots \ldots i_{p}}{ }^{j_{1} \ldots j_{q}} \\
& +\ldots+\left(\partial_{i_{p}} \xi^{k}\right) \mathcal{T}_{i_{1} \ldots i_{p-1} k}{ }^{j_{1} \ldots j_{q}}-\left(\partial_{k} \xi^{j_{1}}\right) \mathcal{T}_{i_{1} \ldots i_{p}}{ }^{k j_{2} \ldots j_{q}} \\
& -\left(\partial_{k} \xi^{j_{2}}\right) \mathcal{T}_{i_{1} \ldots i_{p}}{ }^{j_{1} \ldots j_{q}}-\ldots \\
& -\left(\partial_{k} \xi^{j_{q}}\right) \mathcal{T}_{i_{1} \ldots i_{p}}^{j_{1} \ldots j_{q-1} k}
\end{aligned}
$$

Important properties of the Lie derivative are:

1. $\mathcal{L}_{\xi}(a \Psi)=a \mathcal{L}_{\xi} \Psi$

(Linearity),

2. $\mathcal{L}_{\xi}(\Psi \Phi)=\left(\mathcal{L}_{\xi} \Psi\right) \Phi+\Psi\left(\mathcal{L}_{\xi} \Phi\right)$

(Leibniz rule),

3. $\mathcal{L}_{\xi_{1}}\left(\mathcal{L}_{\xi_{2}} \Psi\right)-\mathcal{L}_{\xi_{1}}\left(\mathcal{L}_{\xi_{2}} \Psi\right)=\mathcal{L}_{\left[\xi_{1}, \xi_{2}\right]} \Psi$

(noncommutativity),

where $\xi_{1}$ and $\xi_{2}$ are two vector fields, and $\left[\xi_{1}, \xi_{2}\right]$ is a new vector field, with components

$$
\left[\xi_{1}, \xi_{2}\right]^{i}=\xi_{1}^{j} \partial_{j} \xi_{2}^{i}-\xi_{2}^{j} \partial_{j} \xi_{1}^{i}
$$

Important particular cases of Lie derivatives we have used are, for a tensor density of weight +1 , as the constitutive tensor:

$$
\begin{aligned}
\mathcal{L}_{\xi} \chi^{i j k l}= & \partial_{p}\left(\xi^{p} \chi^{i j k l}\right)-\left(\partial_{p} \xi^{i}\right) \chi^{p j k l}-\left(\partial_{p} \xi^{j}\right) \chi^{i p k l} \\
& -\left(\partial_{p} \xi^{k}\right) \chi^{i j p l}-\left(\partial_{p} \xi^{l}\right) \chi^{i j k p}
\end{aligned}
$$


for a second order covariant tensor, as for instance the field strength:

$$
\mathcal{L}_{\xi} F_{i j}=\xi^{p}\left(\partial_{p} F_{i j}\right)+\left(\partial_{i} \xi^{p}\right) F_{p j}+\left(\partial_{j} \xi^{p}\right) F_{i p}
$$

for all $\xi$; and for the Levi-Civita symbol:

$$
\mathcal{L}_{\xi} \epsilon^{i j k l} \equiv 0 .
$$

\section{C.3 Differential forms}

A differential $p$-form $\omega$ at a point $x$ of a manifold is a map $\omega: X_{x} \times \cdots \times$ $X_{x} \rightarrow \mathcal{R}$ from a direct product of $p$ times the tangent space $X_{x}$ into the real numbers. The space of differential $p$-forms on the manifold $X$ will be denoted by $\Lambda^{p}(X)$.

If $v_{1}, \cdots, v_{p}$ are $p$ vectors of $X_{x}$, then $\omega\left(v_{1}, \cdots, v_{p}\right)$ is a real number, which is totally antisymmetric under the exchange of vectors $v_{1}, \cdots, v_{p}$, i.e.

$$
\omega\left(v_{1}, \cdots, v_{\alpha}, \cdots, v_{\beta}, \cdots, v_{p}\right)=-\omega\left(v_{1}, \cdots, v_{\beta}, \cdots, v_{\alpha}, \cdots, v_{p}\right) .
$$

The components of $\omega$ with respect to a basis $e_{\alpha}, \alpha, \beta, \ldots=0,1,2,3$ of $X_{x}$ are defined by

$$
\omega_{\alpha_{1} \cdots \alpha_{p}}:=\omega\left(e_{\alpha_{1}}, \cdots, e_{\alpha_{p}}\right) .
$$

In terms of a basis $\vartheta^{\alpha}$ of the cotangent space $X_{x}^{*}$, dual to $e_{\alpha}$, i.e. $\vartheta^{\alpha}\left(e_{\beta}\right)=\delta_{\beta}^{\alpha}$, we have

$$
\omega=\frac{1}{p !} \omega_{\alpha_{1} \cdots \alpha_{p}}(x) \vartheta^{\alpha_{1}} \wedge \cdots \wedge \vartheta^{\alpha_{p}}, \quad \omega_{\alpha_{1} \cdots \alpha_{p}}=\omega_{\left[\alpha_{1} \cdots \alpha_{p}\right]} .
$$

In particular, the basis $e_{\alpha}$ and $\vartheta^{\alpha}$ can be taken as the natural basis associated to a given local coordinate system $x^{i}$, i.e. $e_{\alpha} \rightarrow \partial_{i}$ and $\vartheta^{\alpha} \rightarrow d x^{i}$, with $d x^{i}\left(\partial_{j}\right)=\delta_{j}^{i}$. Then,

$$
\omega=\frac{1}{p !} \omega_{i_{1} \cdots i_{p}}(x) d x^{i_{1}} \wedge \cdots \wedge d x^{i_{p}},
$$

with $\omega_{i_{1} \cdots i_{p}}$ being the coordinate components of $\omega$. It is clear from the antisymmetry property (C.11b) that $p$-forms with $p>n$ vanish identically. Finally, 0 -forms can be defined as being functions from $X$ to the real numbers. 


\section{C.4 Exterior derivative}

The exterior derivative $d$ is a map $d: \Lambda^{p}(X) \rightarrow \Lambda^{p+1}(X)$ which takes $p$-form $\omega$, with components as in (C.12), and produces a $(p+1)$-form $d \omega$, defined by

$$
d \omega:=\frac{1}{p !}\left[\partial_{j} \omega_{i_{1} \cdots i_{p}}\right](x) d x^{j} \wedge d x^{i_{1}} \wedge \cdots \wedge d x^{i_{p}} .
$$

The exterior derivative satisfies the following properties:

1. $d(\omega+\psi)=d \omega+d \psi$

(linearity),

2. $d(\omega \wedge \phi)=d \omega \wedge \phi+(-1)^{p} \omega \wedge d \phi$

((anti-)Leibniz rule),

3. $d(d \omega)=0$

(nilpotency),

for any $\omega, \psi \in \Lambda^{p}(X), \phi \in \Lambda^{q}(X)$.

\section{C.5 Interior product of a vector and a $p$-form}

Given a vector $u$, we define the interior product of $u$ as a map $u\rfloor: \Lambda^{p}(X) \rightarrow$ $\Lambda^{p-1}(X)$, i.e. an operator which decreases the rank of a form. If $\omega \in \Lambda^{p}(X)$ then $u\rfloor \omega$ is the $(p-1)$-form defined by

$$
(u\rfloor \omega)\left(v_{1}, \cdots, v_{p-1}\right):=\omega\left(u, v_{1}, \cdots, v_{p-1}\right),
$$

see (C.9). Additionally, the interior product of any vector with a 0 -form is defined to be zero.

The interior product satisfies:

1. $u\rfloor(\omega+\psi)=u\rfloor \omega+u\rfloor \psi$

(distributivity),

2. $(u+v)\rfloor \omega=u\rfloor \omega+v\rfloor \omega$ (linearity in a vector),

3. $(a u)\rfloor \omega=a(u\rfloor \omega)$ (multiplicative law),

4. $u\rfloor(v\rfloor \omega)=-v\rfloor(u\rfloor \omega)$ (anticommutativity),

5. $\left.u\rfloor(\omega \wedge \phi)=(u\rfloor \omega) \wedge \phi+(-1)^{p} \omega \wedge(u\rfloor \phi\right)$ ((anti-)Leinniz rule).

Explicitly, if $\omega$ has components as in (C.12), then

$$
u\rfloor \omega=\frac{1}{(p-1) !} u^{j} \omega_{j i_{1} \cdots i_{(p-1)}}(x) d x^{i_{1}} \wedge \cdots \wedge d x^{i_{(p-1)}} .
$$




\section{C.6 Lie derivative of a $p$-form}

The Lie derivative of a differential form along a vector field $\xi(x)$ is a map $\mathcal{L}_{\xi}: \Lambda^{p}(X) \rightarrow \Lambda^{p}(X)$, defined by

$$
\left.\left.\mathcal{L}_{\xi} \omega:=\xi\right\rfloor(d \omega)+d(\xi\rfloor \omega\right),
$$

and satisfies the following properties:

1. $\mathcal{L}_{\xi}(d \omega)=d\left(\mathcal{L}_{\xi} \omega\right)$

2. $\mathcal{L}_{\xi}(\omega \wedge \phi)=\left(\mathcal{L}_{\xi} \omega\right) \wedge \phi+\omega \wedge\left(\mathcal{L}_{\xi} \phi\right)$

3. $\left.\mathcal{L}_{(f \xi)} \omega=f \mathcal{L}_{\xi} \omega+d f \wedge(\xi\rfloor \omega\right)$

4. $\mathcal{L}_{\xi_{1}}\left(\mathcal{L}_{\xi_{2}} \omega\right)-\mathcal{L}_{\xi_{2}}\left(\mathcal{L}_{\xi_{1}} \omega\right)=\mathcal{L}_{\left[\xi_{1}, \xi_{2}\right]} \omega$

5. $\left.\left.\left.\mathcal{L}_{\xi_{1}}\left(\xi_{2}\right\rfloor \omega\right)-\xi_{2}\right\rfloor\left(\mathcal{L}_{\left(\xi_{1}\right)} \omega\right)=\left[\xi_{1}, \xi_{2}\right]\right\rfloor \omega$
$(\mathcal{L}$ and $d$ commute $)$

(Leibniz rule),

(rescaled vector),

(non-commutativity),

$(\mathcal{L}$ and $\rfloor$ do not commute $)$

for every $\omega \in \Lambda^{p}(X), \phi \in \Lambda^{q}(X), f \in \Lambda^{0}(X)$, and any vectors $\xi_{1}$ and $\xi_{2}$.

Notice that this definition is consistent with that of the Lie derivative of tensors. For example, if $F$ is a 2 -form with components $F_{i j}$, so that $F:=F_{i j} d x^{i} \wedge d x^{j} / 2$, then

$$
\mathcal{L}_{\xi} F=\frac{1}{2}\left(\mathcal{L}_{\xi} F_{i j}\right) d x^{i} \wedge d x^{j}
$$

\section{C.7 Twisted and untwisted forms}

The differential forms we have considered so far are independent of any coordinate system. However, their components with respect to some coordinates $x^{i}$ are, obviously, coordinate-dependent. In particular, if $\eta$ is a differential form of maximun rank, i.e. $\eta \in \Lambda^{4}(X)$ in our 4-dimensional case, its single coordinate component $\eta_{0123}$, defined by $\eta=\eta_{0123} d x^{0} \wedge d x^{1} \wedge d x^{2} \wedge d x^{3}$ will change sign under a coordinate transformation of opposite orientation (i.e., with negative Jacobian). Consider, for example, $x^{i^{\prime}}:=\left(x^{0}, x^{1}, x^{2},-x^{3}\right)$. Then $\eta_{0^{\prime} 1^{\prime} 2^{\prime} 3^{\prime}}=-\eta_{0123}$. This property has as the consequence that the integral of a $n$-form on a $n$-dimensional manifold (which is defined as the Riemman integral of its single independent component) will change sign under a coordinate transformation changing orientation. In other words, integration 
of normal or untwisted depends on the orientation of the used coordinate system. As a consequence, untwisted forms can be integrated only on manifolds which are orientable.

On the other hand, twisted ${ }^{1}$ forms are defined such that their integral is orientation-independent and are thus possible to integrate on non-orientable manifolds. An twisted $p$-form $\rho$ can be defined in analogously to normal forms with respect to all coordinate systems of the same orientation, but changing sign in a coordinate system of opposite orientation. This means, however, that if $\rho$ is a 4 -form then the sign of its component $\rho_{0123}$ is independent of the orientation of the coordinate system. As a consequence, the integral of $\rho$ on a 4-dimensional region has the same value, independent of the coordinate system in which it is computed. Because of this property, twisted forms are the natural objects used to describe densities, i.e. quantities related to properties defined as integrals over some $p$-dimensional region, since the later should be independent of the orientation of any coordinate system.

\section{C.8 Hodge dual operator}

When a metric $g$ is available, one can immediately define the corresponding Hodge dual operator ${ }^{*}: \Lambda^{p}(X) \rightarrow$ twisted $\Lambda^{n-p}(X)$ and ${ }^{*}:$ twisted $\Lambda^{p}(X) \rightarrow$ $\Lambda^{n-p}(X)$. It is a linear operator, whose action on the respective basis of $\Lambda^{p}(X)$ is given by

$$
\begin{aligned}
& { }^{*} 1=\eta=\frac{1}{4 !} \hat{\epsilon}_{\alpha \beta \gamma \delta} \vartheta^{\alpha} \wedge \vartheta^{\beta} \wedge \vartheta^{\gamma} \wedge \vartheta^{\delta}, \\
& \left.{ }^{*} \vartheta^{\alpha}=e^{\alpha}\right\rfloor \eta=\frac{1}{3 !} g^{\alpha \beta} \hat{\epsilon}_{\beta \gamma \delta \sigma} \vartheta^{\gamma} \wedge \vartheta^{\delta} \wedge \vartheta^{\sigma} \\
& \left.\left.{ }^{*}\left(\vartheta^{\alpha} \wedge \vartheta^{\beta}\right)=e^{\beta}\right\rfloor\left(e^{\alpha}\right\rfloor \eta\right)=\frac{1}{2 !} g^{\alpha \gamma} g^{\beta \delta} \hat{\epsilon}_{\gamma \delta \sigma \rho} \vartheta^{\sigma} \wedge \vartheta^{\rho}, \\
& \left.\left.\left.{ }^{*}\left(\vartheta^{\alpha} \wedge \vartheta^{\beta} \wedge \vartheta^{\gamma}\right)=e^{\gamma}\right\rfloor\left(e^{\beta}\right\rfloor\left(e^{\alpha}\right\rfloor \eta\right)\right)=g^{\alpha \delta} g^{\beta \sigma} g^{\gamma \rho} \hat{\epsilon}_{\delta \sigma \rho \lambda} \vartheta^{\lambda} \\
& \left.\left.\left.\left.{ }^{*}\left(\vartheta^{\alpha} \wedge \vartheta^{\beta} \wedge \vartheta^{\gamma} \wedge \vartheta^{\delta}\right)=e^{\delta}\right\rfloor\left(e^{\gamma}\right\rfloor\left(e^{\beta}\right\rfloor\left(e^{\alpha}\right\rfloor \eta\right)\right)\right) \\
& =g^{\alpha \sigma} g^{\beta \rho} g^{\gamma \lambda} g^{\delta \mu} \hat{\epsilon}_{\sigma \rho \lambda \mu},
\end{aligned}
$$

with $e^{\alpha}:=g^{\alpha \beta} e_{\alpha}$.

The Hodge operator satisfies ${ }^{* *} \omega=(-1)^{p(n-p)+1} \omega$ for $\omega \in \Lambda^{p}(X)$.

\footnotetext{
${ }^{1}$ Sometimes also called odd, impair, or pseudo forms.
} 


\section{Appendix D}

\section{Local Lorentz invariance}

Given a lorentzian metric, we can define the notion of local Lorentz invariance. Let $T^{i_{1} \ldots i_{p}}$ be the contravariant coordinate components of a tensor field and $T^{\alpha_{1} \ldots \alpha_{p}}:=e_{i_{1}}{ }^{\alpha_{1}} \cdots e_{i_{p}}{ }^{\alpha_{p}} T^{i_{1} \ldots i_{p}}$ its frame components with respect to an orthonormal frame $e_{\alpha}=e_{\alpha}^{i} \partial_{i}$. A tensor is said to be local Lorentz invariant at a given point, if its frame components are invariant under a local Lorentz rotation of the orthonormal frame. Similar considerations extend to tensor densities.

There are only two geometrical objects which are numerically invariant under local Lorentz transformations: the Minkowski metric $\eta_{\alpha \beta}$ and the LeviCivita tensor density $\hat{\epsilon}_{\alpha \beta \gamma \delta}$. Thus

$$
\mathcal{T}^{\alpha \beta \gamma \delta}=\phi(x)\left(\eta^{\alpha \gamma} \eta^{\beta \delta}-\eta^{\beta \gamma} \eta^{\alpha \delta}\right)+\alpha(x) \epsilon^{\alpha \beta \gamma \delta}
$$

is the most general form of the frame components of a locally Lorentz invariant contravariant fourth rank tensor with the symmetries $\mathcal{T}^{i j k l}=-\mathcal{T}^{j i k l}=$ $-\mathcal{T}^{i j l k}$. Here $\phi$ and $\alpha$ are scalar and pseudo-scalar fields, respectively. Therefore, back in coodinate components, we find that

$$
\chi^{i j k l}=\phi(x) \sqrt{-g}\left(g^{i k} g^{j l}-g^{j k} g^{i l}\right)+\alpha(x) \epsilon^{i j k l}
$$

is the most general form of a locally Lorentz invariant constitutive tensor allowed in a space with a lorentzian metric. Notice that this constitutive tensor is necessarily symmetric. In other words, any additional asymmetric piece $\sim{ }^{(2)} \chi$ would violate local Lorentz invariance. This was found before in the particular examples of constitutive tensors studied by Nieves and Pal, see section 2.6.1. 


\section{Appendix E}

\section{Computer Algebra}

\section{E.1 Defining the components of the constitu- tive tensor}

The following file is used to define the components of a constitutive tensor.

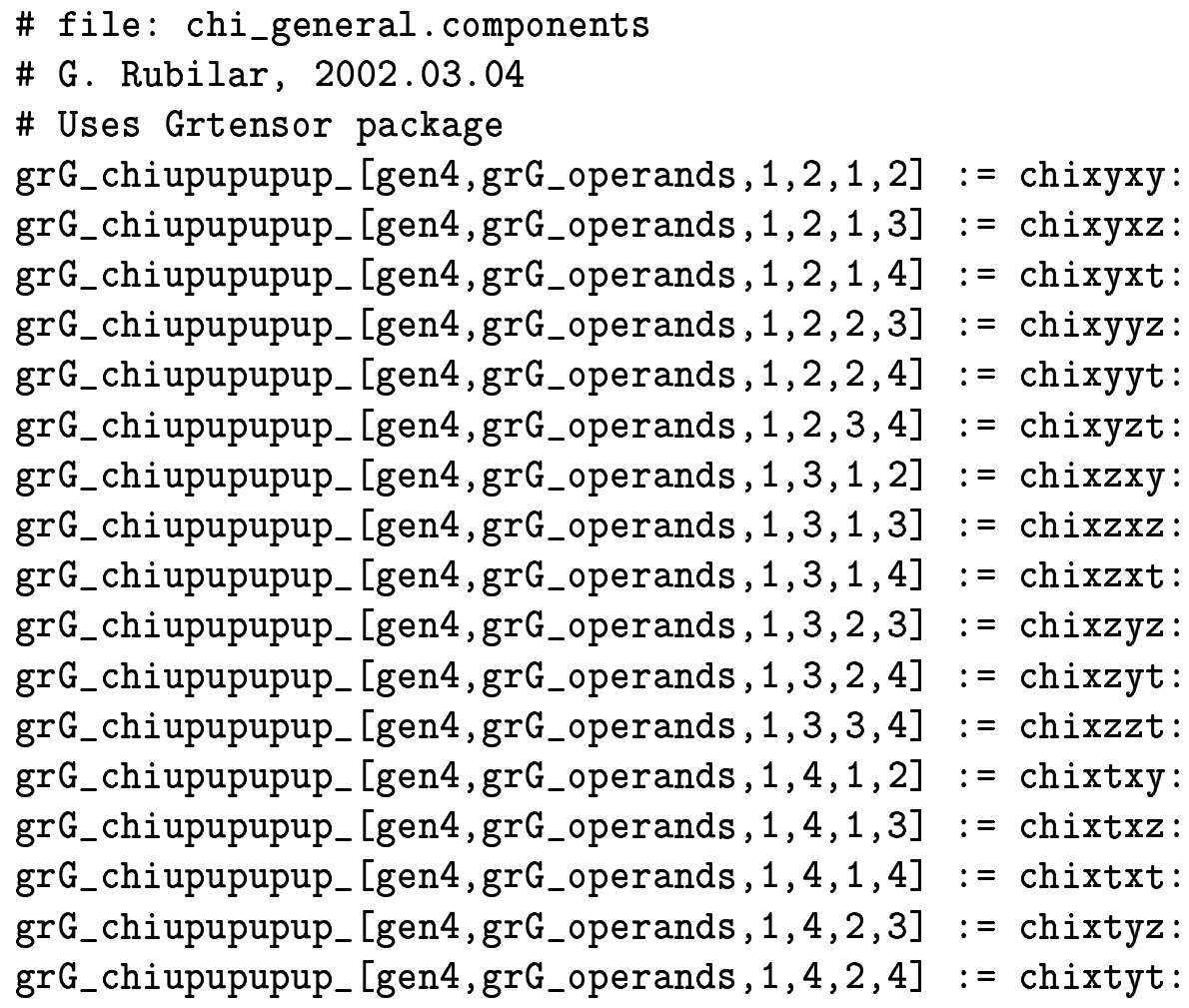




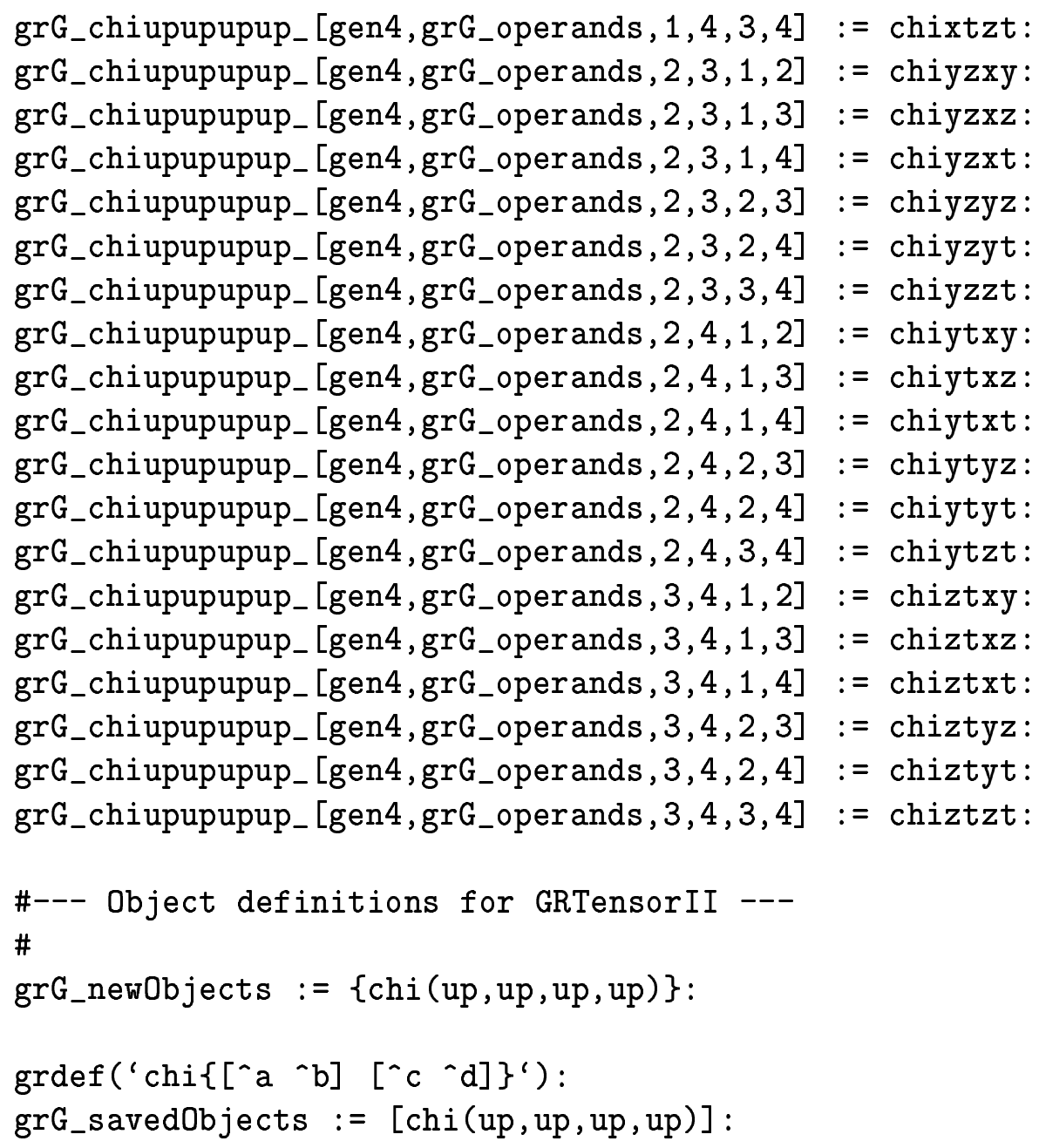

\section{E.2 Computing the Fresnel tensor}

The following program computes the Fresnel tensor according to (3.28) and save its components in the file 'Guuuu.chi_general.components' .

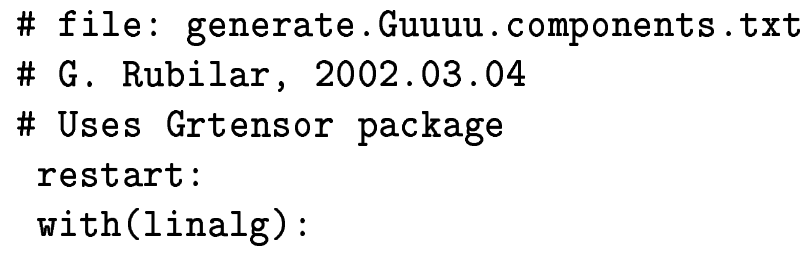


$\operatorname{grtw}()$;

\# Loads a ficticious metric, which is necessary to use GRtensor.

\# It is also used to verify the covariance of the results.

\# If some metric component appears in the final result,

\# then something was wrong.

qload (gen4);

grloadobj (gen4, 'chi_general.components') :

\# grdisplay (chi (up,up, up,up));

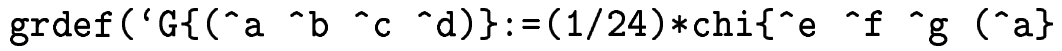

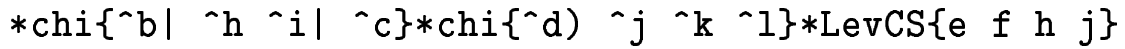

$\left.* \operatorname{LevCS}\{\mathrm{g} i \mathrm{k} 1\}^{\prime}\right)$;

grcalcalter (G(up, up, up, up), simplify);

grdisplay (G (up, up, up, up)) ;

grsaveobj (G (up, up, up, up), 'Guuuu .chi_general.components');

\section{E.2.1 Output}

The ouput file 'Guuuu.chi_general.components' is of the form

grG_Gupupupup_[gen 4, grG_operands $, 1,1,1,1]:=$

-chixyxt*chixtxy*chixzxz+chixtxt*chixyxy*chixzxz

+chixyxt*chixzxy*chixtxz+chixzxt*chixtxy*chixyxz

-chixzxt*chixyxy*chixtxz-chixtxt*chixzxy*chixyxz:

grG_Gupupupup_[gen 4, grG_operands $, 1,1,1,2]:=$

$-1 / 4 * \operatorname{chixyxt} * \operatorname{chixt} x y * \operatorname{chixzyz}-1 / 4 * \operatorname{ch} i x z x z * \operatorname{ch} i x t x y * \operatorname{ch} i x y y t$

$+1 / 4 * \operatorname{chixyxt} * \operatorname{chixzxy} * \operatorname{chixtyz}+1 / 4 * \operatorname{chixzxz} *$ chixyxy $*$ chixtyt

$+1 / 4 * \operatorname{chixzxz} * \operatorname{chixyxy} * \operatorname{ch} i y t x t+1 / 4 * \operatorname{chixzxt} * \operatorname{ch} i x t x y * \operatorname{ch} i x y y z$

$-1 / 4 * \operatorname{chixzxt} * \operatorname{chixy} x y * \operatorname{chixtyz}+1 / 4 * \operatorname{chixzxt} * \operatorname{ch} i \mathrm{ytxy} * \operatorname{ch} i \mathrm{xy} x z$

$-1 / 4 * \operatorname{chixzxt} * \operatorname{chixy} x y * \operatorname{chiytxz}+1 / 4 * \operatorname{chixtxz} * \operatorname{ch} i x z x y * \operatorname{ch} i x y y t$

$-1 / 4 * \operatorname{chixtxz} * \operatorname{chixy} x y * \operatorname{chixzyt}-1 / 4 * \operatorname{chixyxz} * \operatorname{ch} i x z x y * \operatorname{chixtyt}$

$+1 / 4 * \operatorname{chixyxz} * \operatorname{chixtxy} * \operatorname{chixzyt}-1 / 4 * \operatorname{chixtxt} * \operatorname{ch} i x z x y * \operatorname{ch} i x y y z$

$-1 / 4 * \operatorname{chixyxt} * \operatorname{chi}$ txy $*$ chixzxz $-1 / 4 * \operatorname{chixy} x z * \operatorname{ch} i x z x y * \operatorname{ch} i y t x t$

$+1 / 4 *$ chixtxt $*$ chixyxy $*$ chiyzxz $+1 / 4 * \operatorname{ch} i x y x z * \operatorname{ch} i x t x y * \operatorname{ch} i y z x t$

$+1 / 4 * \operatorname{chixtxt} * \operatorname{chixyxy} * \operatorname{chixzyz}-1 / 4 * \operatorname{ch} i x y x t * \operatorname{ch} i x t x y * \operatorname{ch} i y z x z$

$-1 / 4 * \operatorname{chixtxz} * \operatorname{chixyxy} * \operatorname{chiyzxt}+1 / 4 * \operatorname{ch}$ ixyxt $* \operatorname{ch} i x z x y * \operatorname{chi} \mathrm{yt} x z$

$+1 / 4 * \operatorname{chixyxt} * \operatorname{chiyzxy} * \operatorname{ch} i x t x z-1 / 4 * \operatorname{chixtxt} * \operatorname{ch} i y z x y * \operatorname{ch} i x y x z$ : 


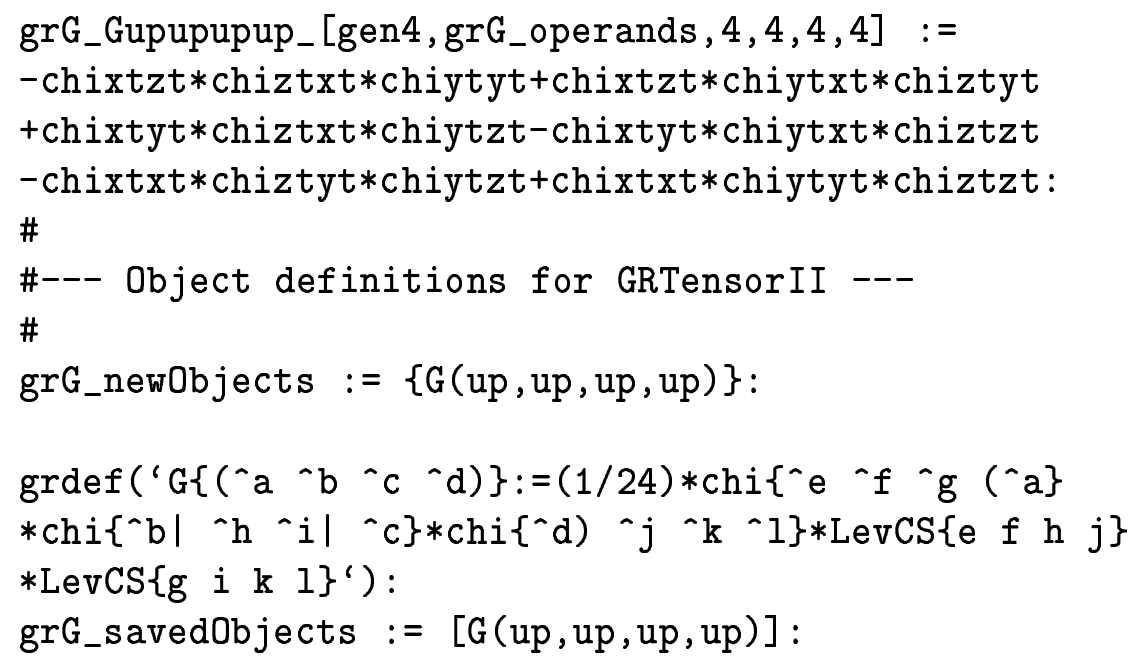

\section{E.3 Checking equations (3.41)}

The following program checks that the Fresnel tensor of a constitutive tensor consisting only of the pieces ${ }^{(2)} \chi$ and ${ }^{(3)} \chi$ vanishes.

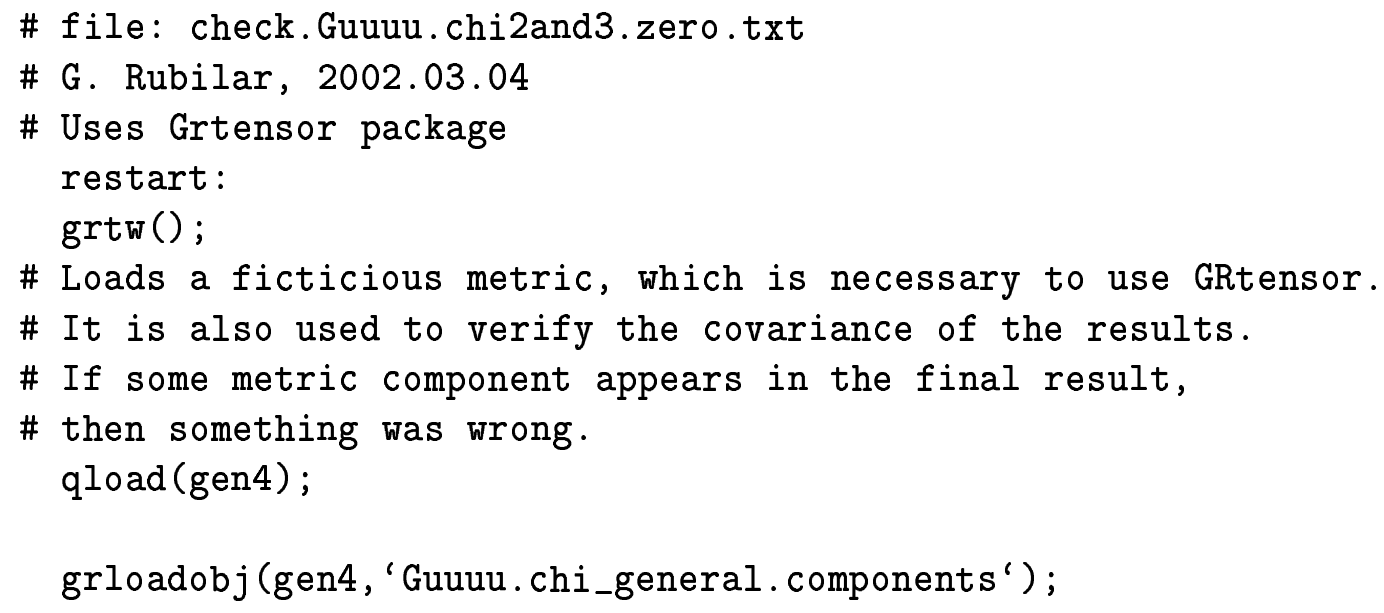




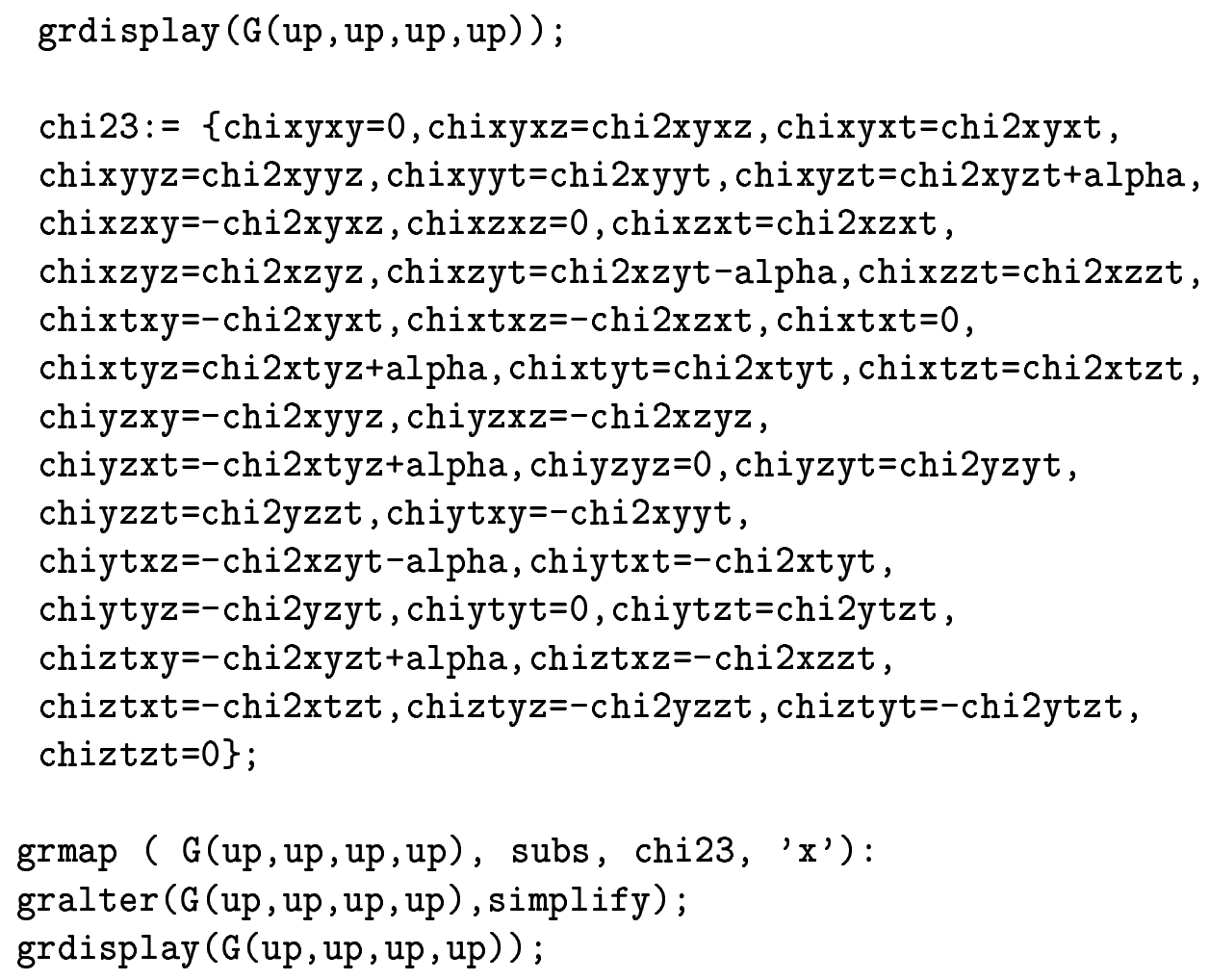

\section{E.4 Defining the 3-dimensional constitutive matrices}

The following file defines the components of the 3-dimensional constitutive matrices $\mathcal{A}, \mathcal{B}, \mathcal{C}$ and $\mathcal{D}$, according to (2.64) and (2.65).

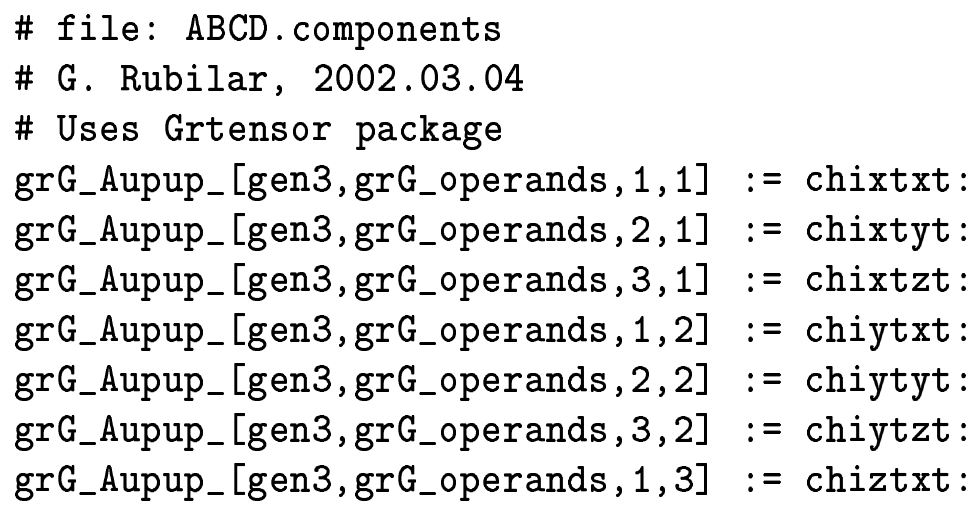




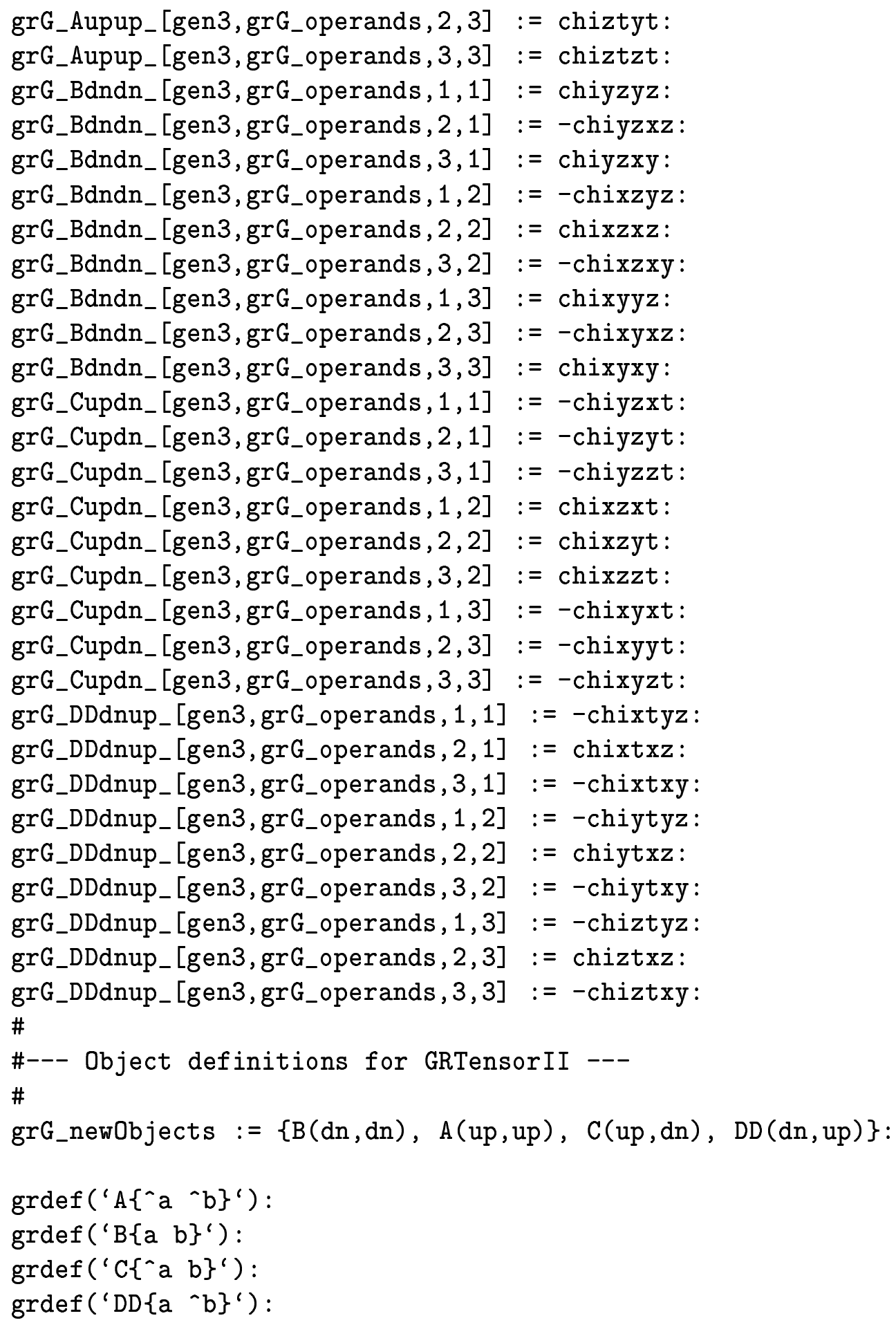


grG_savedObjects := $[A($ up, up $), B(d n, d n), C($ up, dn $), D D(d n, u p)]:$

\section{E.5 Fresnel equation in terms of 3-dimensional constitutive matrices}

The following program computes the Fresnel equation in terms 3-dimensional constitutive matrices $\mathcal{A}, \mathcal{B}, \mathcal{C}$ and $\mathcal{D}$, accordinng to (3.30) and (3.37).

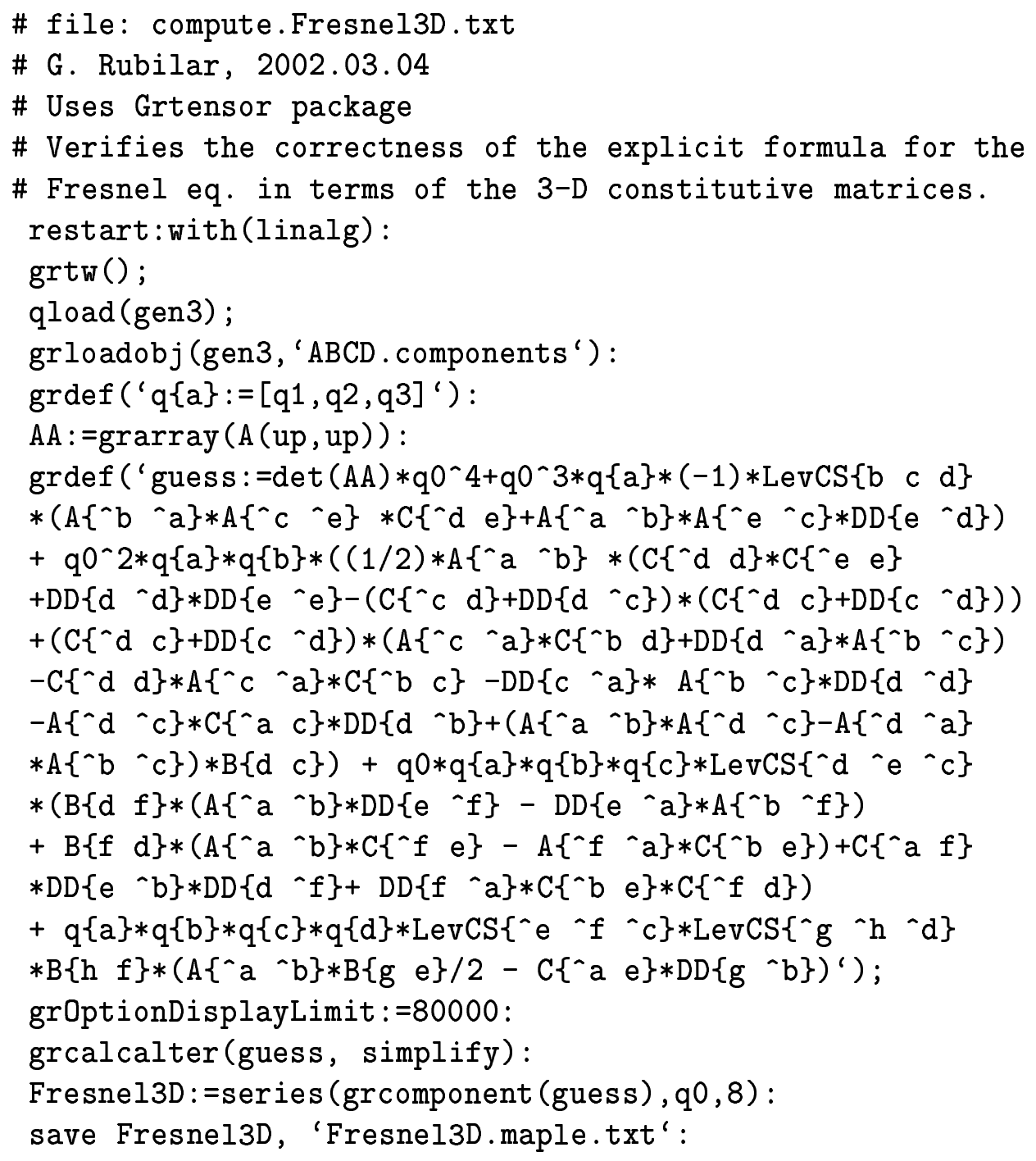




\section{E.6 Checking equations (3.30)-(3.37)}

The following program verifies equations (3.30)-(3.37).

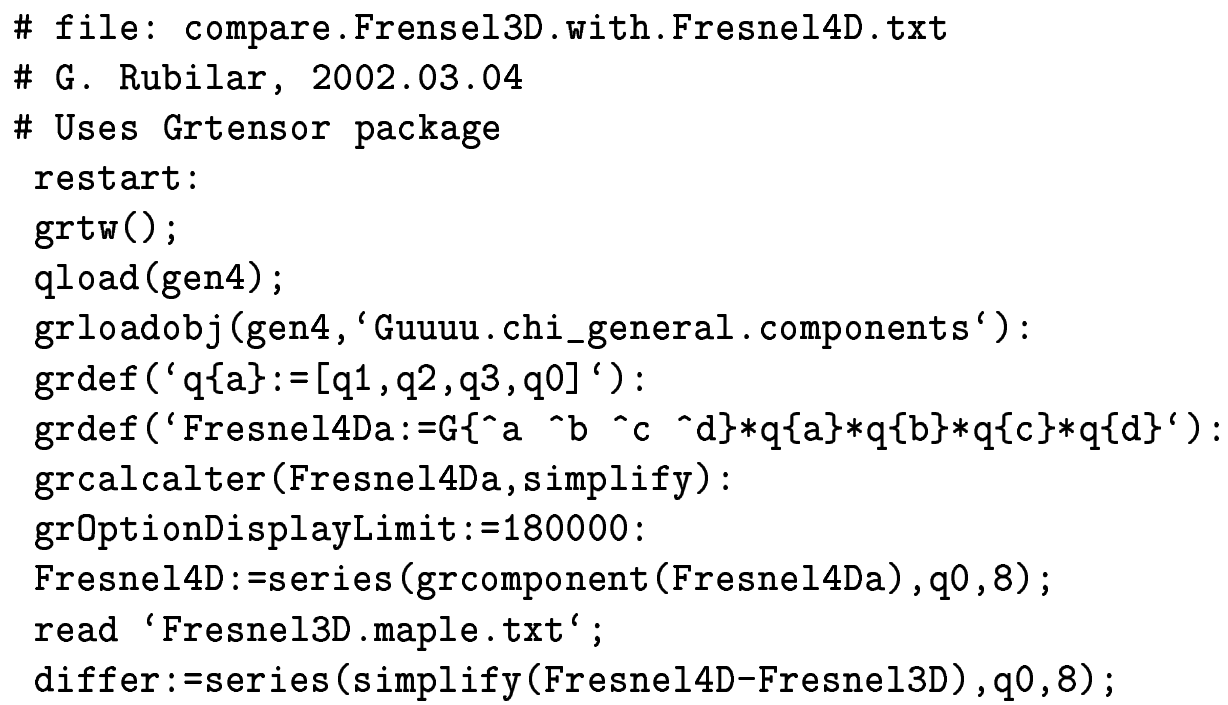




\section{Bibliography}

[1] B. Abbott et al. (D0 Collaboration), A search for heavy pointlike Dirac monopoles, Phys. Rev. Lett. 81 (1998) 524-529.

[2] C. Barceló, S. Liberati and M. Visser, Analogue gravity from field theory normal modes?, Class. Quantum Grav. 18 (2001) 3595-3610.

[3] C. Barceló, S. Liberati and M. Visser, Refringence, field theory, and normal modes, gr-qc/0111059.

[4] F. Bopp, Prinzipien der Elektrodynamik, Z. Physik 169 (1962) 45-52.

[5] M. Born and L. Infeld, Foundations of the new field theory, Proc. Roy. Soc. (London) A 144 (1934) 425-451.

[6] C.H. Brans, Complex 2-form representation of the Einstein equations: The Petrov Type III solutions, J. Math. Phys. 12 (1971) 1616-1619.

[7] C.H. Brans, Complex structures and representations of the Einstein equations, J. Math. Phys. 15 (1974) 1559-1566.

[8] R. Capovilla, T. Jacobson, and J. Dell, General relativity without the metric, Phys. Rev. Lett. 63 (1989) 2325-2328.

[9] R. Capovilla, J. Dell, T. Jacobson and L. Mason, Self-dual 2-forms and gravity, Class. Quantum Grav. 8 (1991) 41-57.

[10] R. Capovilla, J. Dell and T. Jacobson, A pure spin-connection formulation of gravity, Class. Quantum Grav. 8 (1991) 59-73.

[11] S.M. Carroll, G.B. Field, and R. Jackiw, Limits on a Lorentzand parity-violating modification of electrodynamics, Phys. Rev. D41 (1990) 1231-1240. 
[12] R. Chanda, J. Nieves P. Pal, Astrophysical constraints on axion and Majorana couplings, Phys. Rev. D 37 (1988) 2714-2721.

[13] L. Cooper and G.E. Stedman, Axion detection by ring lasers, Phys. Lett. B357 (1995) 464-468. E-Print Archive: hep-ph/9502256.

[14] V.I. Denisov and M.I. Denisov, Verification of Einstein's principle of equivalence using a laser gyroscope in terrestrial conditions, Phys. Rev. D60 (1999) 047301.

[15] I.T. Drummond and S.J. Hathrell, QED vacuum polarization in a background gravitational field and its effect on the velocity of photons, Phys. Rev. D22 (1980) 343-355.

[16] J. Ehlers, Zum Übergang von der Wellenoptik zur geometrischen Optik in der allgemeinen Relativitätstheorie, Z. Naturforsch. 22a (1967) 13281333 .

[17] J. Ellis et al., A search in Gamma-ray burst data for nonconstancy of the velocity of light, Ap. J. $\mathbf{5 3 5}$ (2000) 139-151.

[18] T. Frankel, The Geometry of Physics - An Introduction. Cambridge University Press, Cambridge (1999).

[19] A. Gross and G.F. Rubilar, On the derivation of the spacetime metric from linear electrodynamics, Phys. Lett. A 285 (2001) 267-272. grqc/0103016.

[20] S.A. Gutiérrez, A.L. Dudley, and J.F. Plebanski, Signals and discontinuities in general relativistic nonlinear electrodynamics, J. Math. Phys. 22 (1981) 2835 -2848.

[21] J. Hadamard, Leçons sur la propagation des ondes et les équations de l'hydrodynamique (Hermann: Paris, 1903).

[22] G. Harnett, Metrics and dual operators, J. Math. Phys. 32 (1991) 8491.

[23] G. Harnett, The bivector Clifford algebra and the geometry of Hodge dual operators, J. Phys. A 25 (1992) 5649-5662. 
[24] M.P. Haugan and T.F. Kauffmann, New test of the Einstein equivalence principle and the isotropy of space, Phys. Rev. D52 (1995) 3168-3175.

[25] M. Haugan and C. Lämmerzahl, On the experimental foundations of the Maxwell equations, Ann. Physik (Leipzig) 11 (2000) 507-510.

[26] Y.D. He, Search for a Dirac magnetic monopole in high energy nucleusnucleus collisions, Phys. Rev. Lett. 79 (1997) 3134-3137.

[27] F.W. Hehl, Yu.N. Obukhov, and G.F. Rubilar, Classical electrodynamics: A Tutorial on its Foundations. In Quo vadis geodesia...? Festschrift for Erik W. Grafarend, F. Krumm and V.S. Schwarze (eds.) Univ. Stuttgart, ISSN 0933-2839 (1999) pp. 171-184. E-print Archive: physics/9907046.

[28] F. W. Hehl, Yu.N. Obukhov, G. F. Rubilar, Spacetime metric from linear eletrodynamics II, Annalen der Physik 11 (2000) Spec. Iss. SI 71 - SI 78. E-print Archive: gr-qc/9911096.

[29] F.W. Hehl and Yu.N. Obukhov, How does the electromagnetic field couple to gravity, in particular to metric, nonmetricity, torsion, and curvature?, In "Testing Relativistic Gravity in Space: Gyroscopes, Clocks, Interferometers ...", Proceedings of the 220th Heraeus-Seminar, 22 - 27 August 1999 in Bad Honnef, C. Lämmerzahl et al. (eds.). Springer, Berlin (2000). E-print Archive: gr-qc/0001010.

[30] F.W. Hehl and Yu.N. Obukhov, On the energy-momentum current of the electromagnetic field in a pre-metric axiomatic approach. I. E-print Archive: gr-qc/0103020.

[31] F.W. Hehl, Yu.N. Obukhov, Foundations of classical electrodynamics (Birkhäuser: Boston, MA, 2002) To be published.

[32] F. W. Hehl, Y. Obukhov, G.F. Rubilar, On a possible new type of a $T$ odd skewon field linked to electromagnetism, Proceedings of the 1st Mexican Meeting on Mathematical and Experimental Physics, held at El Colegio Nacional, Mexico City, 10 to 14 September, 2001. E-print Archive: gr-qc/0203096.

[33] W. Heisenberg and H. Euler, Folgerungen aus der Dirac'schen Theorie des Positrons, Z. Phys. 98 (1936) 714 - 732. 
[34] H.S. Ibarguen, A. Garcia, and J. Plebanski, Signals in nonlinear electrodynamics invariant under duality rotations, J. Math. Phys. 30 (1989) $2689-2691$.

[35] C. Itzykson and J.-B. Zuber, Quantum field theory, New York, McGraw Hill (1985).

[36] A.Z. Jadczyk, Electromagnetic permeability of the vacuum and lightcone structure, Bull. Acad. Pol. Sci., Sér. sci. phys. et astr. 27 (1979) $91-94$.

[37] G.R. Kalbfleisch et al., Improved experimental limits on the production of magnetic monopoles, Phys. Rev. Lett. 85 (2000) 5292-5295.

[38] W. Kaminsky: Experimental and phenomenological aspects of circular birefringence and related properties in transparent crystals. Reports on Progress in Physics 63 (2000) 1575-1640.

[39] R.M. Kiehn, G.P. Kiehn, and J.B. Roberds, Parity and time-reversal symmetry breaking, singular solutions, and Fresnel surfaces, Phys. Rev. A43 (1991) 5665-5671.

[40] R.M. Kiehn, Chirality and helicity in terms of topological spin and topological torsion, physics/0101101.

[41] V.A: Kostelecký and M. Mewes, Cosmological constraints on Lorentz violation in electrodynamics, Phys. Rev. Lett. 87 (2001) 251304 (4 pages).

[42] F. Kottler, Maxwell'sche Gleichungen und Metrik, Sitzungsber. Akademie Wien, IIa, 131 (1922) 119-146.

[43] H.F. Kremer, Fresnel's equation in general relativity, J. Math. Phys. 8 (1967) 1197-1199.

[44] C. Lämmerzahl et al., Reasons for the electromagnetic field to obey Maxwell's equations. Preprint, University of Konstanz, August 1999.

[45] L. Landau and E. Lifshitz, Electrodynamics of continuous media. Pergamon Press, Oxford (1960). 
[46] U. Leonhardt, Space-time geometry of quantum dielectrics, Phys. Rev. A 62 (2000) 012111.

[47] U. Leonhardt and P. Piwnicki, Relativistic effects of light in moving media with extremely low group velocity, Phys. Rev. Lett. 84 (2000) $822-825 ; 85$ (2000) 5253.

[48] A. Lichnerowicz, Relativity theory and mathematical physics, in: "Astrofisica e cosmologia gravitazione quanti e relatività", Centenario di Einstein (Giunti Barbera: Firenze, 1979).

[49] I.V. Lindell, A.H. Sihvola, S.A. Tretyakov and A.J. Viitanen, Electromagnetic waves in chiral and bi-isotropic media, Artec House, Boston (1994).

[50] A. Lue, L. Wang and M. Kamionkowski, Cosmological signature of new parity-violating interactions, Phys. Rev. Lett. 83 (1999) 1506-1509.

[51] B. Mashhoon, Influence of gravitation on the propagation of electromagnetic radiation, Phys. Rev. D11 (1975) 2679-2684.

[52] J. Manzano and R. Montemayor, Propagation of light in a gravitational background, Phys. Rev. D56 (1997) 6378-6387.

[53] S. Mohanty and A.R. Prasanna, Photon propagation in Einstein and higher derivative gravity, Nucl. Phys. B526 (1998) 501-508.

[54] C.W. Misner, K.S. Thorne and J.A. Wheleer, Gravitation, W.H. Freeman and Company, San Francisco (1973).

[55] W.-T. Ni, Equivalence principles and electromagnetism, Phys. Rev. Lett. 38 (1977) 301-304.

[56] W.-T. Ni et al., Search for an axionlike spin coupling using a paramagnetic salt with a dc squid, Phys. Rev. Lett. 82 (1999) 2439-2442.

[57] J.F. Nieves and P.B. Pal, $P$ and $C P$-odd terms in the photon self-energy within a medium, Phys. Rev. D39 (1989) 652-659.

[58] J.F. Nieves and P.B. Pal, The third electromagnetic constant of an isotropic medium, Am. J. Phys. 62 (1994) 207-216. 
[59] E.B. Norman, J.N. Bahcall, Improved limit on charge conservation derived from GA-71 solar neutrino experiments, Phys. Rev. D53 (1996) 4086-4088.

[60] M. Novello, V.A. De Lorenci, J.M. Salim, and R. Klippert, Geometrical aspects of light propagation in nonlinear electrodynamics, Phys. Rev. D61 (2000) 045001. E-Print Archive: gr-qc/9911085.

[61] Yu.N. Obukhov and S.I. Tertychniy, Vacuum Einstein equations in terms of curvature forms, Class. Quantum Grav. 13 (1996) 1623-1640.

[62] Yu.N. Obukhov, T. Fukui, G. F. Rubilar, Wave propagation in linear electrodynamics, Phys. Rev. D 62 (2000) 044050. E-print Archive: grqc/0005018.

[63] Yu.N. Obukhov and F.W. Hehl, Space-time metric from linear electrodynamics, Phys. Lett. B458 (1999) 466-470. E-print Archive grqc/9904067.

[64] Yu.N. Obukhov and G.F. Rubilar, Fresnel analysis of the wave propagation in nonlinear electrodynamics, Phys. Rev. D (2002), to appear. E-print Archive: gr-qc/0204028.

[65] T.H. O'Dell, The electrodynamics of magneto-electric media, Selected of monographs on selected topics in solid state physics. Editor E.P. Wohlfarth. North-Holland, Amsterdam (1970).

[66] R.D. Peccei and H.R. Quinn, CP Conservation in the presence of pxseudoparticles, Phys. Rev. Lett. 38 (1977) 1440-1443.

[67] R. Penrose and W. Rindler, Spinors and space-time, Vol 2, Cambridge Univ. Press, Cambridge (1986).

[68] A. Peres, Electromagnetism, geometry, and the equivalence principle, Ann. Phys. (NY) 19 (1962) 279-286.

[69] C. Piron and D.J. Moore, New aspects of field theory, Turk. J. Phys. 19 (1995) 202-216.

[70] J. Plebański, Electromagnetic waves in gravitational fields, Phys. Rev. 118 (1960) 1396-1408. 
[71] E.J. Post: The constitutive map and some of its ramifications. Annals of Physics (NY) 71 (1972) 497-518.

[72] E.J. Post, Formal Structure of Electromagnetics - General Covariance and Electromagnetics. North Holland, Amsterdam (1962) and Dover, Mineola, New York (1997).

[73] A.R. Prasanna, Maxwell's equations in Riemann-Cartan space $U_{4}$, Phys. Lett. A54 (1975) 17 -18.

[74] R.A. Puntigam, C. Lämmerzahl and F.W. Hehl, Maxwell's theory on a post-Riemannian spacetime and the equivalence principle, Class. Quantum Grav. 14 (1997) 1347-1356.

[75] R. de Ritis, M. Lavorgna, and C. Stornaiolo, Geometric optics in a Riemann-Cartan spacetime, Phys. Lett. A98 (1983) 411-413.

[76] G.F. Rubilar., Y.N. Obukhov and F.W. Hehl, General covariant Fresnel equation and the emergence of the light cone structure in premetric electrodynamics, Int. J. Mod. Phys. D (2002), to appear. E-print Archive: gr-qc/0109012.

[77] M. Schönberg, Electromagnetism and gravitation, Rivista Brasileira de Fisica 1 (1971) 91-122.

[78] J.A. Schouten, Tensor Analysis for Physicists. 2nd ed.. Dover, Mineola, New York (1989).

[79] E. Schrödinger, Space-Time structure. Reprinted with corrections, Cambridge University Press, Cambridge (1960).

[80] R. Schützhold, G. Plunien and G. Soff, Dielectric black hole analogs, Phys. Rev. Lett. 88 (2002) 061101 (4 pages).

[81] L.L. Smalley, On the extension of geometric optics from Riemannian to Riemann-Cartan spacetime, Phys. Lett. A117 (1986) 267-269.

[82] G.V. Skrotskii, The influence of gravitation on the propagation of light, Sov. Phys. Doklady 2 (1957) 226-229. 
[83] J. Stachel, Covariant formulation of the Cauchy problem in generalized electrodynamics and general relativity, Acta Phys. Polon. 35 (1969) 689 -709 .

[84] G.E. Stedman, Ring-laser tests of fundamental physics and geophysics, Rep. Prog. Phys. 60 (1997) 615-688.

[85] R.I. Steinberg et al., Experimental test of charge conservation and the stability of the electron, Phys. Rev. 12 (1975) 2582-2586.

[86] R.U. Sexl and H.K. Urbantke, Gravitation und Kosmologie: Eine Einführung in die Allgemeine Relativitätstheorie, 4th rev. ed., Spektrum Akad. Verlag, Heidelberg (1995) p. 50.

[87] J.L. Synge, Relativity: The General Theory, North Holland (1960).

[88] I.E. Tamm, Relativistic crystaloptics in relation with the geometry of bi-quadratic form, J. of Russ. Phys.-Chem. Soc. 57 (1925) 209 -214 (in Russian). [Reprinted in: I.E. Tamm, Collected Papers (Nauka: Moscow, 1975) vol. 1, p. 33-61 (in Russian)].

[89] V. Tapia, Renormalizable conformally invariant model for the gravitational field, Class. Quantum Grav. 13 (1996) 3261-3267.

[90] B. Tellegen, The gyrator, a new network element Philips Res. Rep. 3 (1948) 81-101; Philips Technical Review 18 (1956/57) 120-124. Reprinted in H.B.G. Casimir and S. Gradstein (eds.) An Anthology of Philips Research. Philips' Gloeilampenfabrieken, Eindhoven (1966) pp.186-190.

[91] G. 't Hooft, A chiral alternative to the vierbein field in general relativity, Nucl. Phys. B357 (1991) 211-221.

[92] R.A. Toupin, Elasticity and electro-magnetics, in: Non-Linear Continuum Theories, C.I.M.E. Conference, Bressanone, Italy 1965. C. Truesdell and G. Grioli, coordinators. Pp.206-342.

[93] Tretyakov et al., Reply to comment on 'Reflection and transmition by a uniaxial bi-anisotropic slab under normal incidence of plane waves, J. Phys. D 32 (1999) 2705-2706. 
[94] H. Urbantke, A quasi-metric associated with SU(2) Yang-Mills field, Acta Phys. Austriaca Suppl. XIX (1978) 875-816.

[95] H. Urbantke, On integrability properties of SU(2) Yang-Mills fields. I. Infinitesimal part, J. Math. Phys. 25 (1984) 2321-2324.

[96] K. van Bibber and D. Kinion, Review of Dark-Matter axion experiments, Nucl. Phys. (Proc. Suppl.) 91 (2001) 376-383.

[97] D. van Dantzig, The fundamental equations of electromagnetism, independent of metrical geometry, Proc. Cambr. Phil. Soc., 30 (1934) 421-427.

[98] M. Visser, Comment on "Relativistic effects of light in moving media with extremely low group velocity", Phys. Rev. Lett., 85 (2000) 5252 (1 page).

[99] M. Visser, C. Barceló and S. Liberati, Analogue models of and for gravity, gr-qc/0111111.

[100] A.M. Volkov, A.A. Izmest'ev, and G.V. Skrotskii, The propagation of electromagnetic waves in a Riemannian space, Sov. Phys. JETP 32 (1971) 686-689 [ZhETF 59 (1970) 1254-1261 (in Russian)].

[101] C. Wang, Mathematical Principles of Mechanics and Electromagnetism, Part B: Electromagnetism and Gravitation, Plenum Press, New York (1979).

[102] W.S. Weiglhofer and A. Lakhtakia, Further comments on the nonexistence of linear non-reciprocal bi-isotropic media, J. Phys. A: Math. Gen. 31 (1998) 1113-1114.

[103] W.S. Weiglhofer and A. Lakhtakia, The Post constraint revisited, Arch. Elektron. Übetrag. 52 (1998) 276.

[104] S. Weinberg, A new light boson? Phys. Rev. Lett. 40 (1978) 223-226.

[105] H. Weyl, Raum, Zeit, Materie, 4th edition. Springer, Berlin (1921).

[106] F. Wilczek, Problem of strong $P$ and $T$ invariance in the presence of instantons, Phys. Rev. Lett. 40 (1978) 279-282. 



\section{Acknowledgements}

Many people have contributed to my formation as a physicist in the course of the my stay in Cologne. First of all I would like to thank Prof. Dr. F.W. Hehl for his support and for the discussions and joint work on the subject of this thesis. I also thank Dr. Y. Obukhov (Moscow) for the joint work, and the members of the gravity group at Cologne for their help and kindness. This work could not have been possible without the continuous support and love of my wife Alejandra. Finally, I would like to thank the German Academic Exchange Servie (DAAD) for the financial support which made possible my stay in Germany. 


\section{Erklärung}

Ich versichere, dass ich die von mir vorgelegte Dissertation selbständig und ohne unzulässige Hilfe angefertigt, die benutzten Quellen und Hilfsmittel vollständig angegeben und die Stellen der Arbeit - einschliesslich Tabellen, Karten und Abbildungen -, die anderen Werken im Wortlaut oder im Sinn nach entnommen sind, in jedem Einzelfall als Entlehnung kenntlich gemacht habe; dass diese Dissertation noch keiner anderen Fakultät oder Universität zur Prüfung vorgelen hat; dass sie - abgesehen von unten angegebenen Teilpublikationen - noch nicht veröffentlicht worden ist sowie, dass ich eine solche Veröffentlichung vor Abschluss des Promotionsverfahrens nicht vornehmen werde.

Die Bestimmungen der geltenden Promotionsordnung sind mit bekannt. Die von mir vorgelegte Dissertation ist von Hern Prof. Dr. F.W. Hehl betreut worden.

Teilveröffentlichungen:

$\underline{\text { Refereed: }}$

1. F. W. Hehl, Y. N. Obukhov, G. F. Rubilar, Spacetime metric from linear electrodynamics II, Annalen der Physik 11 (2000) Spec. Iss. SI 71 - SI 78. E-print Archive: gr-qc/9911096.

2. Y. Obukhov, T. Fukui and G.F. Rubilar, Wave propagation in linear electrodynamics, Phys. Rev. D 62044050 (2000). E-print Archive: gr-qc/0005018.

3. A. Gross and G.F. Rubilar, On the derivation of the spacetime metric from linear electrodynamics, Phys. Lett. A 285 (2001) 267-272. Eprint Archive: gr-qc/0103016. 
4. G.F. Rubilar, Y. Obukhov and F. W. Hehl, General covariant Fresnel equation and the emergence of the light cone structure in pre-metric electrodynamics, Int. J. Mod. Phys. D (2002), to appear. E-print Archive: gr-qc/0109012.

5. Yu.N. Obukhov and G.F. Rubilar, Fresnel analysis of the wave propagation in nonlinear electrodynamics, Phys. Rev. D (2002), to appear. E-print Archive: gr-qc/0204028.

Non-Refereed:

1. F.W. Hehl, Y. Obukhov and G.F. Rubilar, Classical electrodynamics: A Tutorial on its Foundations, in: Quo vadis geodesia...? Festschrift for Erik W. Grafarend, F. Krumm and V.S. Schwarze (eds.) Univ. Stuttgart, ISSN 0933-2839 (1999) pp. 171-184. E-print Archive: physics $/ 9907046$.

2. F.W. Hehl, Y. Obukhov and G.F. Rubilar, Light propagation in generally covariant electrodynamics and the Fresnel equation, Invited talk given at Journeés Relativistes, University College Dublin, Sept. 2001. Int. J. Mod. Phys. (2002), to appear. E-print Archive: gr-qc/0203105

3. F. W. Hehl, Y. Obukhov, G.F. Rubilar, On a possible new type of a $T$ odd skewon field linked to electromagnetism, Proceedings of the 1st Mexican Meeting on Mathematical and Experimental Physics, held at El Colegio Nacional, Mexico City, 10 to 14 September, 2001. E-print Archive: gr-qc/0203096.

Köln, im März 2002. 


\section{Lebenslauf}

Vornamen: Guillermo Francisco

Familiennamen: Rubilar Alegría

Geburtsdatum: $\quad 29.01 .1973$

Geburtsort: $\quad$ Concepción, Chile

Eltern: $\quad$ Isolina Alegría Zuñiga

Francisco Rubilar Cabezas

Familienstand: verheiratet

Staatangehörigkeit: chilenisch

Schulausbildung: $\quad$ März 1978 - Dezember 1985: Grundschule der Stadt Talcahuano: Escuela E-504.

März 1986 - Dezember 1989: Gymnasium der Stadt Concepción: Liceo Enrique Molina Garmendia.

Dezember 1989: Abitur.

Hochschulausbildung: März 1990 - Dezember 1994: Studium der Physik an der Universität Concepción, Concepción, Chile.

März 1994: Abschluss "Licenciatura en Física", Universität Concepción, Concepción, Chile.

November 1997: "Magister en Física", Universität Concepción, Concepción, Chile.

Oktober 1998 - Juni 2002 Stipendiat Deutscher

Akademischer Austauschdienst (DAAD). 